\title{
The effective temperatures of carbon-rich stars st, $^{\star}$
}

\author{
J. Bergeat, A. Knapik, and B. Rutily \\ Centre de Recherche Astronomique de Lyon, UMR 5574 du CNRS, Observatoire de Lyon, 9 avenue Charles André, \\ 69561 St-Genis-Laval Cedex, France
}

Received 20 July 2000 / Accepted 22 December 2000

\begin{abstract}
We evaluate effective temperatures of 390 carbon-rich stars. The interstellar extinction on their lines of sights was determined and circumstellar contributions derived. The intrinsic (dereddened) spectral energy distributions (SEDs) are classified into 14 photometric groups $\left(\mathrm{HC}_{i}, \mathrm{CV}_{j}\right.$ and $\mathrm{SCV}$ with $i=0,5$ and $\left.j=1,7\right)$. The new scale of effective temperatures proposed here is calibrated on the 54 angular diameters (measured on 52 stars) available at present from lunar occultations and interferometry. The brightness distribution on stellar discs and its influence on diameter evaluations are discussed. The effective temperatures directly deduced from those diameters correlate with the classification into photometric groups, despite the large error bars on diameters. The main parameter of our photometric classification is thus effective temperature. Our photometric $\langle k\rangle^{1 / 2}$ coefficients are shown to be angular diameters on a relative scale for a given photometric group, (more precisely for a given effective temperature). The angular diameters are consistent with the photometric data previously shown to be consistent with the true parallaxes from HIPPARCOS observations (Knapik, et al. 1998, Sect. 6). Provisional effective temperatures, as constrained by a successful comparison of dereddened SEDs from observations to model atmosphere predictions, are in good agreement with the values directly calculated from the observed angular diameters and with those deduced from five selected intrinsic color indices. These three approaches were used to calibrate a reference angular diameter $\Phi_{0}$ and the associated coefficient $C_{T_{\text {eff }}}$. The effective temperature proposed for each star is the arithmetic mean of two estimates, one ("bolometric") from a reference integrated flux $F_{0}$, the other ("spectral") from calibrated color indices which are representative of SED shapes. Effective temperatures for about 390 carbon stars are provided on this new homogeneous scale, together with values for some stars classified with oxygen-type SEDs with a total of 438 SEDs (410 stars) studied. Apparent bolometric magnitudes are given. Objects with strong infrared excesses and optically thick circumstellar dust shells are discussed separately. The new effective temperature scale is shown to be compatible and (statistically) consistent with the sample of direct values from the observed angular diameters. The effective temperatures are confirmed to be higher than the mean color temperatures (from 140 to $440 \mathrm{~K}$ ). They are in good agreement with the published estimates from the infrared flux method for $T_{\text {eff }} \geq 3170 \mathrm{~K}$, while an increasing discrepancy is observed toward lower temperatures. As an illustration of the efficiency of the photometric classification and effective temperature scale, the $\mathrm{C} / \mathrm{O}$ ratios and the Merrill-Sanford (M-S) band intensities are investigated. It is shown that the maximum value, mean value and dispersion of $\mathrm{C} / \mathrm{O}$ increase along the photometric $\mathrm{CV}$-sequence, i.e. with decreasing effective temperature. The M-S bands of $\mathrm{SiC}_{2}$ are shown to have a transition from "none" to "strong" at $T_{\text {eff }} \simeq(2800 \pm 150) \mathrm{K}$. Simultaneously, with decreasing effective temperature, the mean C/O ratio increases from 1.04 to 1.36, the transition in $\mathrm{SiC}_{2}$ strength occurring while $1.07 \leq \mathrm{C} / \mathrm{O} \leq 1.18$.
\end{abstract}

Key words. stars: AGB \& post-AGB - stars: carbon - stars: fundamental parameters - stars: variables: general circumstellar matter

\section{Introduction}

This paper deals with the determination of the effective temperatures of chemically peculiar red giants with

\footnotetext{
Send offprint requests to: J. Bergeat

* This research has made use of the Simbad database operated at CDS, Strasbourg, France.

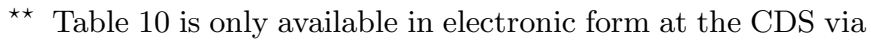
anonymous ftp to

cdsarc.u-strasbg.fr (130.79.128.5) or via

http://cdsweb.u-strasbg.fr/cgi-bin/qcat?J/A+A/369/178
}

carbon overabundance, and of their apparent bolometric magnitudes, in order to define a homogeneous scale of effective temperatures for all carbon giants and related objects. The BaII stars and carbon-rich stars we classified as having oxygen-rich types of SEDs will be attributed (Knapik et al. 2000) effective temperatures from published calibrations (Ridgway et al. 1980; Perrin et al. 1998; Richichi et al. 1999; Houdashelt et al. 2000). For carbon and SC-stars, such calibrations are presently missing and we intend to establish them. 
Mendoza \& Johnson (1965), first attempted such a study using a method previously used for oxygen-rich cool giants. Having obtained the multicolor ( $U B V R I J K L)$ photometry of 44 stars, including 39 carbon-rich giants in the Arizona system, they used the $(R+I)-(J+K)$ index calibrated from (oxygen-rich) K and M-stars. Even if the derived values look reasonable, this approach is not adequate since the opacities and SEDs of carbon stars appreciably differ from those of oxygen-rich giants (e.g. Bergeat et al. 1976a and references therein). In addition, the interstellar extinction was assumed negligible for their sample of (mainly) bright carbon stars. Substantial reddenings are however present on the lines of sight to some of their stars. Mendoza \& Johnson (1965) found that most of their effective temperatures were larger than the vibrational temperatures derived from spectroscopy (Bouigue 1954; Wyller 1960; McKellar \& Buscombe 1948).

The multicolor photometry of 29 cool carbon stars was used by Bergeat et al. (1976b), who tried to take into account the influence of the circumstellar dust shells they found around most of their stars (the deduced optical depths were however small except for a few Miras and "infrared" carbon stars). They made no correction for interstellar reddening and their temperatures are systematically lower than previous ones. They showed that a few carbon stars do exhibit vibrational temperatures (e.g. $3350 \mathrm{~K}$ for $\mathrm{C} 4038=\mathrm{T}$ Lyr a J-star with strong $\mathrm{C}_{2}$ bands) much higher than their effective temperatures $(2400 \mathrm{~K}$ for $\mathrm{T}$ Lyr). The temperatures from spectroscopy appear often badly affected by strong blends, which are influenced by abundances in the atmosphere. They are not reliable indicators of effective temperature.

Tsuji (1981a, 1981b) applied the infrared flux method (IRFM) of Blackwell \& Shallis (1977) and Blackwell et al. (1980) to carbon stars. More recently, temperatures and angular diameters were derived for $114 \mathrm{~F}-\mathrm{M}$ stars using the IRF method (Blackwell et al. 1990), which consists of determining the ratio of the bolometric (i.e. integrated) flux to the net flux in a selected photometric band, and then comparing the result to the predictions from model atmospheres. For the carbon stars, Tsuji selected the $L$-band $\left(\lambda=3.5 \mu \mathrm{m} ; R_{L}\right.$ ratio $)$ since it is unaffected by strong bandheads observed elsewhere, except for the HCN + $\mathrm{C}_{2} \mathrm{H}_{2}$ features (centered at $\lambda=3.1 \mu \mathrm{m}$, at the edge of the filter bandpass). The author applied empirical corrections to compensate and corrected for the estimated effect of interstellar extinction. He mentioned a good agreement with the values from five angular diameters observed during lunar occultations (see however the discussions in Sects. 14 and 16).

Ridgway et al. (1981) established an effective temperature scale for cool carbon stars based on the angular diameters from lunar occultations. Their effective temperatures were systematically higher than the mean color temperatures deduced from photometry, by nearly $300 \mathrm{~K}$, a result which is found again in the present analysis (Sect. 15). Many additional measurements became available since then, especially from interferometry
(Dyck et al. 1996b; van Belle et al. 1997; van Belle \& Thompson 1999), increasing the number of usable angular diameters up to 54 (52 stars), including several carbon Miras at known phases. Some were observed in the visible but the largest body of data lies in the near infrared. The consistency of the collected diameters with independent photometry is established through

- a provisional calibration of five color indices against "direct" effective temperatures (Sect. 7);

- the relation shown in Fig. 5 for a given photometric group or for sets of groups, between the observed angular diameters and the values of our $\langle k\rangle^{1 / 2}$ coefficient from photometric analysis (Sect. 8).

This paper aims to build a new effective temperature scale for carbon stars, consistent with both observed angular diameters and analyzed photometry as a substitute for detailed SEDs. Both data are compared to the predictions of stellar model atmospheres. The first classification of the carbon-rich giants in discrete photometric groups was proposed by Knapik \& Bergeat (1997, hereafter Paper I), Bergeat et al. (1999 hereafter Paper II, for the hot carbon stars), and Knapik et al. (1999 hereafter Paper III, for the coolest carbon variables and SC stars). The new homogeneous scale for effective temperatures we propose here is independent of the group classification developed in Papers I to III.

We briefly summarize the results of our method of analysis of observed SEDs and the classification scheme into photometric groups (Sect. 2). We describe our approach in Sect. 3. Integrated (bolometric) fluxes are then derived for nearly 400 carbon stars (Sect. 4). The available angular diameters are compiled and their relation to effective temperatures are discussed (Sect. 5). The good correlation of effective temperatures, as directly deduced from observed angular diameters, with our classification in CV-groups is shown and discussed (Sect. 6). We provide a provisional calibration of five selected color indices in terms of these direct temperatures (Sect. 7). Tight relations are established between the observed angular diameters and the $\langle k\rangle^{1 / 2}$ coefficients from photometry (Sect. 8). The case of C $5928=$ TX Psc is revisited (Sect. 9) and the predictions from model atmospheres are successfully compared to the mean SEDs of our photometric groups (Sect. 10). Reference angular diameters $\Phi_{0}$ and effective temperatures coefficients $C_{T_{\text {eff }}}$ are then defined and calibrated (Sect. 11). A homogeneous scale for effective temperatures of nearly 400 carbon stars is finally proposed (Sect. 12). Carbon stars with strong thermal (grain) emission in the IR, are discussed separately (Sect. 13). The temperatures on the new scale are checked against the 54 direct values (Sect. 14). They are compared to the mean color temperatures (Sect. 15) and to the values derived from the IRF method (Sect. 16). We prove the efficiency of the photometric classification and effective temperature scale on the $\mathrm{C} / \mathrm{O}$ abundance ratio (Sect. 17) and the intensity of the Merrill-Sanford bands of $\mathrm{SiC}_{2}$ (Sect. 18). 


\section{The analysis of the observed SEDs}

The first classification of the carbon-rich giants in discrete photometric groups was proposed by the authors (Papers I, II and III), independent of any spectral classification. We have provided the classification in a CV $i$-group (intrinsic SEDs from $i=1$ (the earliest) to $i=6$ (the latest), in Paper I) and the amount of interstellar extinction, $A_{J}$, in the $J$-filter. The color excess is $E(B-V) \simeq 1.15 A_{J}$ for the mean extinction law of the diffuse interstellar medium (Mathis 1990) which was shown to be relevant. A good agreement of color excesses compared to field values from the maps found in the literature was found. For other stars (about 10\%), variable circumstellar extinction was noted. No gap was observed and discrete CV $i$-groups are adopted here only for convenience. The main features of this new pair method (fully described in Paper I and Sect. 2 of Paper III), are

- the simultaneous use of the whole spectral range from UV to IR (up to 17 wavelengths);

- the derivation of the relevant CV $i$-group and selective extinction of dust through a linear fit;

- $E(B-V) \simeq 0.02-0.03$ as a detection threshold;

- the derivation of a $\langle k\rangle^{1 / 2}$ quantity, an angular diameter on a relative scale (Sect. 8).

The $\langle k\rangle^{1 / 2}$ coefficient also showed a correlation with true parallaxes (derived from the HIPPARCOS data: ESA 1997, henceforth called ESA) expected for stars of a given range in linear diameters (see Fig. 3 and Sect. 6 of Knapik et al. 1998; also Knapik et al. 2000).

Paper I concentrated on carbon stars with small or moderate amplitudes of variations (namely Lb or SR variables). Paper II extended to HC stars (i.e. stars classified as early $\mathrm{R}$ based on their spectra). Finally, the carbon Miras, and the CS and SC stars, were included (Paper III). A seventh group, CV7, was added on the cool edge, and an additional SCV-group for SC-stars filled the gap to S-types SEDs. A sequence S-SC-CS-C was indicated. A carbon sequence of 13 groups ( $\mathrm{HC} 0$ to $\mathrm{HC} 5$ followed by CV1 to CV7) was obtained, which is a sequence of decreasing effective temperatures, as shown hereafter. The fourteenth (SCV) group remains outside this sequence. We also considered three sequences (sg for supergiants, $\mathrm{g}$ for giants and $\mathrm{d}$ for dwarfs) of photometric groups for oxygen-rich stars labelled with reference spectral types, such as F8d, K2g or G8sg. These SEDs were used by Bergeat \& Knapik (1997) in their analysis of BaII stars and by Bergeat et al. (Paper II) in that of very hot carbon stars, including RCB variables and HdCs or carbon Cepheids, and of the carbon-rich RV Tau-star, AC Her. The junction between carbon and oxygen SEDs does occur in the mid G-types.

The circumstellar extinction and its time variations were also studied in Papers II and III for stars exhibiting substantial infrared excesses attributed to thermal emission from circumstellar grains. The RCB variables (Paper II) and the cool carbon Miras and IRAS carbon stars (Paper III) were investigated. Several conclusions were derived about the optical properties of circumstellar grains and/or the circumstellar geometry. The phase dependence of extinction and dust clearing near maximum light in carbon Miras were documented. From every analyzed SED, five "products" are obtained, viz.

- the classification in 14 carbon photometric groups $\left(\mathrm{HC}_{i}, \mathrm{CV}_{j}, \mathrm{SCV} ; i=0,5 j=1,7\right)$ and nearly 30 oxygen ones (with the sg, g and d luminosity classes);

- the amount of interstellar and/or circumstellar (selective) extinction in the $J$-filter, i.e. $A_{J}$;

- the dereddened SED from ultraviolet to infrared;

- the integrated flux derived from integration of this SED (Sect. 4) or from calibrated bolometric corrections (Knapik et al. 2000);

- the quantity $\langle k\rangle^{1 / 2}$ which is a "photometric" angular diameter on a relative scale (Sect. 8 and Fig. 5).

\section{The approach adopted in the present study}

A classical approach in spectral analyses is to obtain a detailed agreement between observed and predicted spectra "line by line" over a few selected spectral ranges which are assumed to be representative. Supposedly, detailed observations contain sufficient information to determine simultaneously the effective temperature, surface gravity, the detailed chemical (including isotopic) composition and the microturbulence velocity (usually a few $\mathrm{km} \mathrm{s}^{-1}$ ), all of these being entry parameters in model atmospheres (see e.g. Lambert et al. 1986) who made use of unpublished models in their detailed study of 30 cool carbon stars). It should be emphasized, however, that they used initial estimates of effective temperatures obtained by other methods. The above-mentioned simultaneous determination of parameters is difficult when no initial guess is available. Having derived a solution, the question is whether observations could be satisfactorily modeled using a different set of values.

Our approach is different. Since the line and band intensities are known to be sensitive to chemical composition, they are not used to constrain the effective temperature. Local differences in molecular band intensities may occur in two SEDs otherwise globally similar. More specifically, two stars in a same photometric group may show similar intrinsic SEDs on the whole spectral range we use, and exhibit substantial differences in some molecular band intensities over limited spectral regions. Energy blocked in very opaque bandheads is redistributed in less opaque spectral ranges, i.e. strong blanketing is present. Our new effective temperature scale (Sect. 12) relies on both the spectral shape (color indices) and the energy budget (bolometric fluxes).

As a result, stars with nearly the same effective temperature (eventually gravity) and quite different abundance ratios $\left(\mathrm{C} / \mathrm{O},{ }^{12} \mathrm{C} /{ }^{13} \mathrm{C} \ldots\right.$, or $\left.\mathrm{O} / \mathrm{H}, \mathrm{C} / \mathrm{H} . ..\right)$ may coexist in the same photometric group. This is the case of $\mathrm{C} 4038=\mathrm{T}$ Lyr $(\mathrm{CV} 6, E(B-V)=0.24)$ with very strong 
Table 1. The comparison of the integrated fluxes $\left(\right.$ in $\left.\mathrm{Wcm}^{-2}\right)$ as derived from the spectrophotometric data $\left(F_{\mathrm{sp}}, n\right.$ number of points), and from multicolor photometry ( $F_{\mathrm{ph} 1}, n 1$ number of bands); $F_{\mathrm{ph} 2}$ is the flux obtained without the two "continuum" points $[0,78]$ and $[1,08]$ from Baumert (1972). We thus have $n_{2}=n_{1}-2$. The ratios $r_{1}=F_{\mathrm{sp}} / F_{\mathrm{ph} 1}$ and $r_{2}=F_{\mathrm{sp}} / F_{\mathrm{ph} 2}$ are close to unity, and $m_{\mathrm{bol}}$ is the apparent bolometric magnitude. The stars are classified into two categories (I and II, not luminosity classes!). Ext. is the color excess $E(B-V)$. Phase data and J-star classification are quoted in the "Remarks" column

\begin{tabular}{|c|c|c|c|c|c|c|c|c|c|c|c|c|c|}
\hline $\mathrm{C}$ & GCVS & Gr. & Ext. & $n$ & $F_{\mathrm{sp}}$ & $F_{\mathrm{ph} 1}$ & $F_{\mathrm{ph} 2}$ & $n_{1}$ & $r_{1}$ & $r_{2}$ & $m_{\text {bol }}$ & Remarks & Cat. \\
\hline 5928 & TX Psc & $\mathrm{CV} 2$ & 0.03 & 383 & $2.7210^{-13}$ & $2.8310^{-13}$ & $2.7410^{-13}$ & 19 & 0.96 & 0.99 & 2.41 & & I \\
\hline 5976 & WZ Cas & $\mathrm{CV} 2$ & 0.34 & 286 & $9.1910^{-14}$ & $1.0410^{-13}$ & $1.0110^{-13}$ & 18 & 0.89 & 0.91 & 3.59 & ${ }^{*} \mathrm{~J}, \phi=0.6$ & II \\
\hline 3283 & Y CVn & CV5 & 0.00 & 437 & $2.6610^{-13}$ & $3.0210^{-13}$ & $2.9410^{-13}$ & 21 & 0.88 & 0.90 & 2.43 & $* \mathrm{~J}$ & II \\
\hline 1316 & UU Aur & CV4 & 0.09 & 288 & $2.9610^{-13}$ & $2.8910^{-13}$ & $2.8110^{-13}$ & 18 & 1.02 & 1.05 & 2.32 & & I \\
\hline 5425 & RV Cyg & CV5 & 0.39 & 401 & $1.0310^{-13}$ & $1.1110^{-13}$ & $1.0510^{-13}$ & 14 & 0.93 & 0.98 & 3.46 & & I \\
\hline 198 & Z Psc & $\mathrm{CV} 2$ & 0.04 & 372 & $7.3510^{-14}$ & $7.7110^{-14}$ & $7.5310^{-14}$ & 17 & 0.95 & 0.98 & 3.93 & & I \\
\hline 36 & VX And & CV6 & 0.00 & 499 & $4.8910^{-14}$ & $5.7510^{-14}$ & $5.7410^{-14}$ & 17 & 0.85 & 0.85 & 4.27 & $* \mathrm{~J}$ & II \\
\hline 3652 & VCrB & CV7 & 0.00 & 273 & $2.9210^{-14}$ & $3.2410^{-14}$ & $3.1910^{-14}$ & 16 & 0.90 & 0.92 & 4.83 & Mira, $\phi=0.15$ & II \\
\hline
\end{tabular}

Ballik-Ramsay $\mathrm{C}_{2}$ bands between 1.4 and $2.0 \mu \mathrm{m}$ (e.g. Bergeat et al. 1976a) and strong $\mathrm{HCN}$ and $\mathrm{C}_{2} \mathrm{H}_{2}$ bands near $3.1 \mu \mathrm{m}$ (Johnson \& Méndez 1970). It is a ${ }^{13} \mathrm{C}-$ rich i.e. J-type star (Bouigue 1954). For a given group and effective temperature, those band intensities do increase with increasing $\mathrm{C} / \mathrm{O}$ ratios and decreasing ${ }^{12} \mathrm{C} /{ }^{13} \mathrm{C}$ ratios.

\section{Multicolor photometry and spectrophotometry: The integrated fluxes}

Here, we examine the accuracy of the integrated fluxes derived from the multicolor photometry collected in Papers I to III. We compare them to the values obtained from the detailed spectrophotometry available on a sample of eight stars and estimate a correction factor ( $r$; Sect. 4.1). Then we test for accuracy the integrations of spectrophotometric SEDs through blackbody SEDs integration (Sect. 4.2).

\subsection{Reference SEDs and the r-correction factor}

We have been able to find among the brightest carbon variables, eight stars with detailed multi-wavelength spectrophotometry. A few "holes" on limited spectral intervals were filled in by interpolation, making use of the available information on molecular features intensities (essentially those of $\mathrm{CN}, \mathrm{CO}, \mathrm{C}_{2}, \mathrm{HCN}$ and $\mathrm{C}_{2} \mathrm{H}_{2}$ ), either in the considered star or in a close analogue. As in Papers I to III, pieces of SEDs have to be assembled with the best possible continuity. Unfortunately, no extensive simultaneous spectrophotometry is presently available on any carbon star. For instance, the visible and infrared sections were observed at different epochs, by different observers and equipment, in various observing conditions, which limits our approach, but we feel confident that correction factors can be estimated in this way. Every spectrophotometric SED was required to be consistent with the adopted photometric SED. For an irregular variable or a semi-regular one without period and phase, this means that the net flux level in both SEDs should not differ significantly over too large a spectral domain. For Miras and semi-regulars with known periods and phases, the large amplitude of their variations complicates the analysis. This explains why we could select so few stars, with none in the HCs, SCV, CV1 and CV3 photometric groups. Further studies should fill the gaps. The eight selected stars do have measured angular diameters.

The main sources of the photometric data can be found in Papers I to III, as well as

- ultraviolet: Johnson et al. (1986, 1995), Johnson \& Luttermoser (1987);

- visible and near infrared: Fäy \& Honeycutt (1972), Baumert (1972), Fäy et al. (1974), Burnashov (1979), Avetysian et al. (1981), Gunn \& Stryker (1983), Oganessian et al. (1985), Eglitis (1988, 1990);

- infrared: Bergeat et al (1976a), Johnson \& Méndez (1970), Merrill \& Stein (1976), Noguchi et al. (1977), Goebel et al. (1978, 1980, 1981), Forrest et al. (1981), Olnon \& Raimond (IRAS Science Team, 1986), Volk \& Cohen (1989), Lázaro et al. (1994), Aoki et al. (1998).

For Mira C3652 = V CrB, we used the unpublished 1.4-4.0 $\mu \mathrm{m}$ spectrophotometry observed with a CVF by Bergeat \& Garnier at phase $\phi \simeq 0.15$ in 1986. Their fluxes are close to those of the photometry used in the solution adopted at the same phase in Paper III. It is in stark contrast to the data of Goebel et al. (1981) at phase $\phi \simeq 0.6$, a cooler SED with much stronger molecular bands. Finally, eight spectrophotometric SEDs with between 273 and 499 wavelengths were obtained (Fig. 1, Table 1). Natural logarithms were used in abscissae together with $\lambda F_{\lambda}^{0}$ in Watt $\mathrm{cm}^{-2}$ as ordinates. The area below SED is the integrated flux in Watt $\mathrm{cm}^{-2}$, with no additional factor required. The integrated net fluxes as obtained from these " $n$-wavelengths" distributions are quoted as $F_{\mathrm{sp}}$ while the values from the photometric points $\left(n_{1}=14\right.$ to 21$)$ are given as $F_{\mathrm{ph} 1}$. With one exception $(\mathrm{C} 1316=\mathrm{UU}$ Aur), the former are slightly smaller than the latter. We have attempted a second comparison with the photometric integrated fluxes $F_{\mathrm{ph} 2}$ obtained without the fluxes from the narrow-band photometry of Baumert (1972) at $\lambda=0.78 \mu \mathrm{m}$ and $\lambda=1.08 \mu \mathrm{m}$, i.e. with $n_{2}=n_{1}-2$ wavelengths. These pseudo-continuum points may induce some overestimate of the integrated fluxes $F_{\mathrm{ph} 1}$. Better agreement is noted with $F_{\mathrm{ph} 2}$ but the spectrophotometric integrated fluxes remain on average slightly smaller than the 

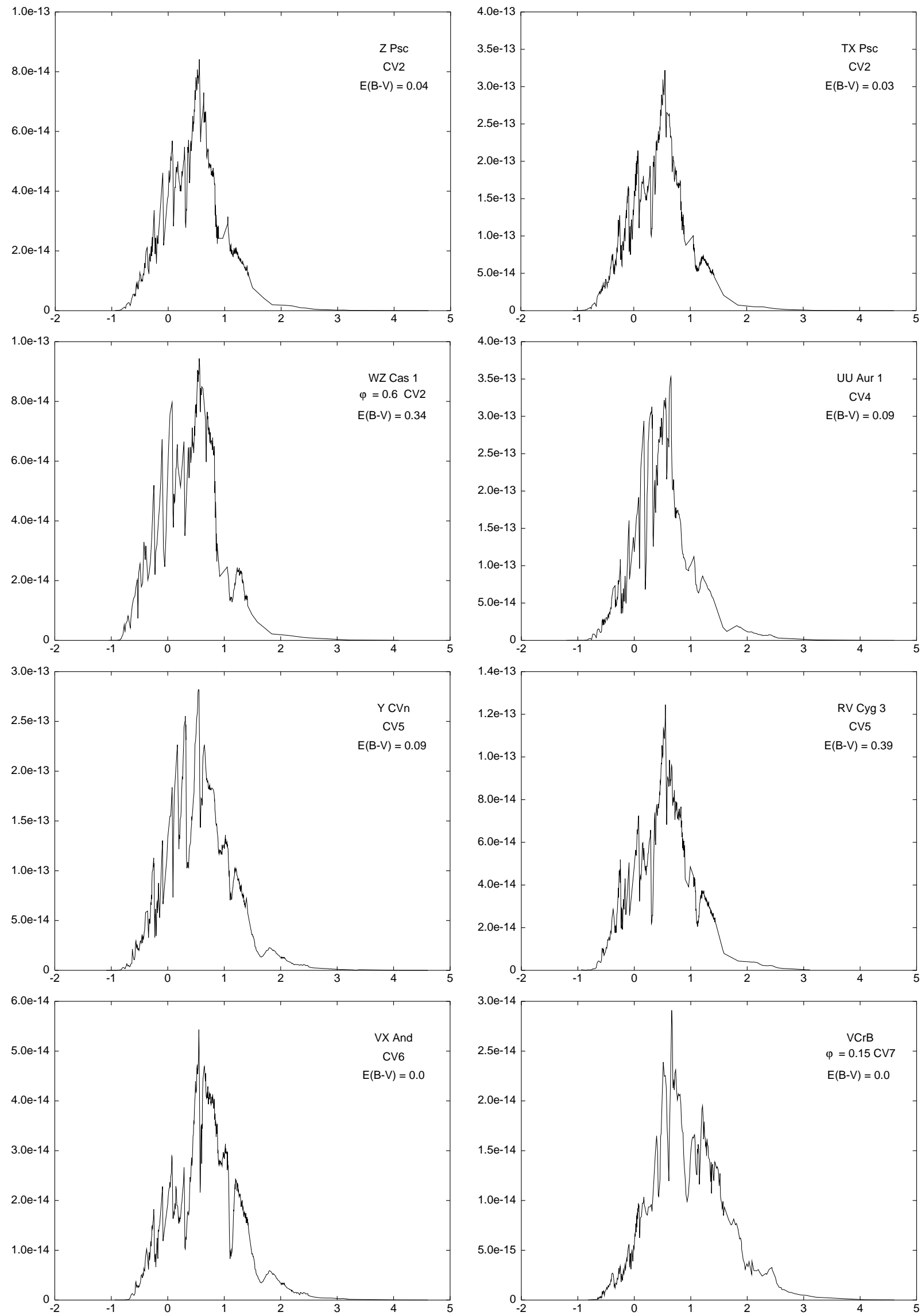

Fig. 1. Dereddened SEDs spectrophotometry of the eight carbon stars selected for comparison of integrated fluxes from spectrophotometry and from multicolor photometry (abscissae: $\ln \lambda_{\mu \mathrm{m}}$, ordinates: $\lambda F_{\lambda}^{0}$ in Watt $\mathrm{cm}^{-2}$; see text for details) 


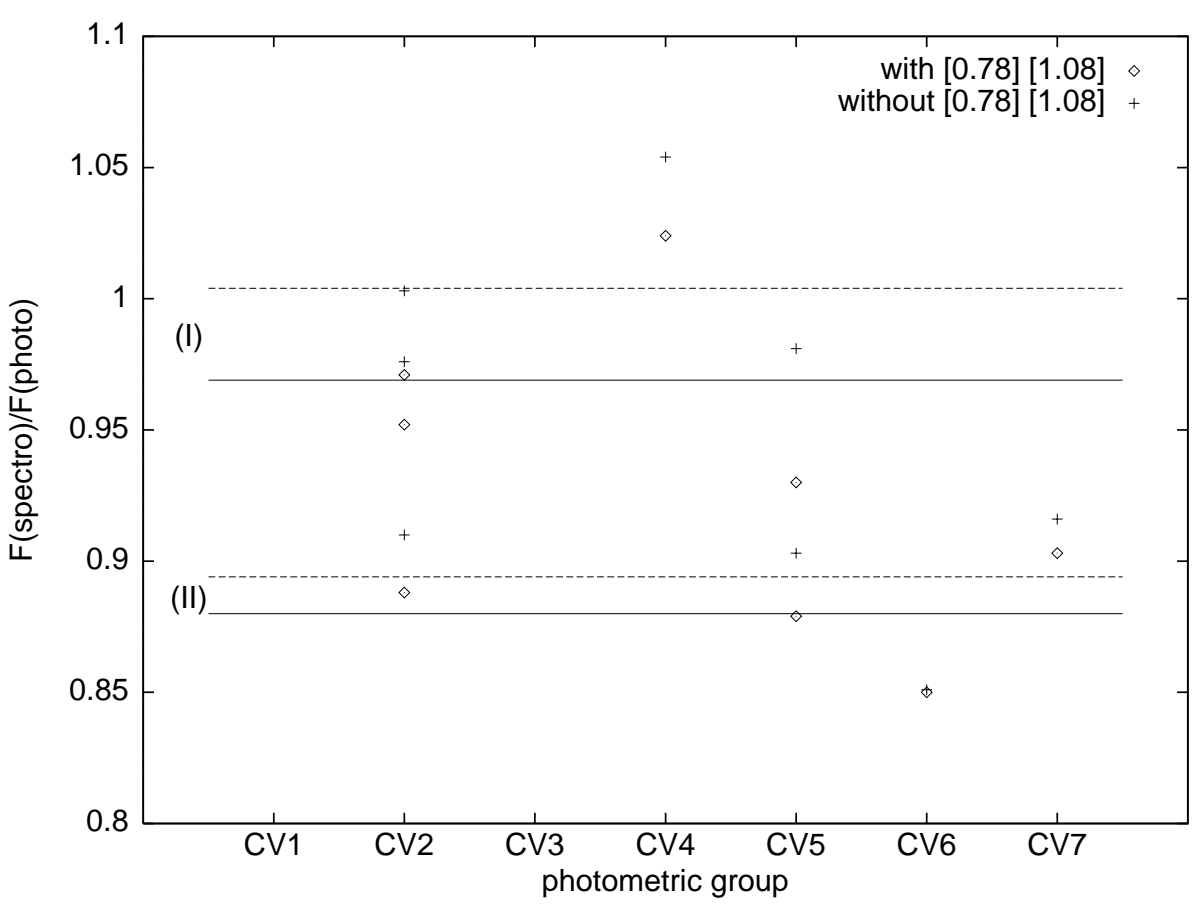

Fig. 2. The $r_{1}$ and $r_{2}$-ratios, as defined by Eqs. (1) and (2), against the CV-group, for the 8 stars of Table 1 . Averaged correction factors adopted for samples I and II are also shown

photometric ones. The quoted values are however close to each other, as shown by the ratios in Table 1, viz.

$$
\begin{aligned}
& r_{1}=F_{\mathrm{sp}} / F_{\mathrm{ph} 1} \\
& r_{2}=F_{\mathrm{sp}} / F_{\mathrm{ph} 2} .
\end{aligned}
$$

Figure 2 shows that there is no correlation between those ratios and the photometric groups. The ratios are systematically lower by nearly $10 \%$ for the J-stars (WZ Cas, Y CVn and VX And) and also for the cool (CV7) Mira V $\mathrm{CrB}$. The many spectral lines of molecules with ${ }^{13} \mathrm{C}$ are slightly shifted in wavelength with respect to their ${ }^{12} \mathrm{C}$ analogues. Thus, for a given total column density of carbon and similar photometric SEDs as described by the photometric groups, the spectral intervals with strong absorptions are enlarged, and fluxes outside them are augmented due to blanketing. The integrated flux is then overestimated when derived from photometry secured outside the strong molecular bandheads. It is also presumably the case for the coolest (CV7) variables whose spectra are affected by numerous strong bands. As shown in Fig. 1, the region of the maximum in the SED of $\mathrm{V} \mathrm{CrB}$, where $\lambda F_{\lambda}^{0} \geq 10^{-14} \mathrm{~W} \mathrm{~cm}^{-2}$, is actually depressed by very strong bands. For $\phi \simeq 0.6$, the measurements of Goebel et al. (1981) exhibit even larger absorptions with very strong bandheads.

According to the above analysis, we distinguished between categories, numbered I ("usual" CV-stars) and II (J-type and very cool CVs, such as CV7-Miras). Making use of the data in Table 1, we deduced the mean correction factors for category I, namely

$\left\langle r_{1}\right\rangle=0.97 \pm 0.03$ $\left\langle r_{2}\right\rangle=1.00 \pm 0.03$

and for category II,

$\left\langle r_{1}\right\rangle=0.88 \pm 0.02$

$\left\langle r_{2}\right\rangle=0.89 \pm 0.02$.

From a careful scrutiny of the available partial spectrophotometry of a few HC4 and HC5 stars, we decided to apply the above correction factors also to them all, and to SCVstars as well. For the hotter HC0 to HC3-stars, no correction was attempted $\left(\left\langle r_{1}\right\rangle=\left\langle r_{2}\right\rangle=1\right)$ since the molecular bands are typically much fainter in their spectra. Future efforts should be made to obtain compatible spectrophotometric SEDs for all those groups.

Equivalent spectrophotometric integrated fluxes were thus derived for about 320 well-documented stars following the above-mentioned guidelines. The sample was extended to nearly 400 stars through bolometric corrections (Knapik et al. 2000) for various bandpasses ( $V, J, H$, $K,[1.08], \ldots)$, as calibrated against color indices such as $(J-K)_{0},(V-K)_{0} \ldots$ The apparent bolometric magnitudes were then derived from the $F_{\mathrm{sp}}$ fluxes, adopting $F=2.5010^{-12} \mathrm{~W} \mathrm{~cm}^{-2}$ for $m_{\mathrm{bol}}=0$. They are also quoted in Table 1 for our eight reference stars. Finally, we have calculated the integrated net flux $F_{0}$ the star would radiate if it had $[1.08]_{0}=0$. The relation used was

$F_{0}=F_{\mathrm{sp}} 10^{0.4[1.08]_{0}}$

where $[1.08]_{0}$ is the magnitude after correction for selective extinction (Papers I to III). These latter fluxes are used in forthcoming Eq. (37), to calculate the values of the $C_{T_{\text {eff }}}$ 
coefficient. They are required since the $\langle k\rangle^{1 / 2}$ coefficients from photometry (angular diameters on a relative scale in Eq. (47)) are also referred to unity for a $[1.08]_{0}=0$ star.

\subsection{The accuracy of the integrations of spectrophotometric SEDs}

The sources of errors when integrating the spectrophotometric SEDs are essentially the limited wavelength coverage (typically 0.3 to $100 \mu \mathrm{m}$ ) and the accuracy of the used algorithm. We evaluated these by comparison to theoretical fluxes from Stephan's law. For temperatures lower than $3500 \mathrm{~K}$, the departure did not exceed $1 \%$ for $n=273$ to 499 spectral points, which is the case of the eight stars in Table 1. It may reach a few percent at temperatures higher than $4500 \mathrm{~K}$, specific of $\mathrm{HC}$ stars.

\section{Angular diameters and effective temperatures}

\subsection{The observed angular diameters}

When reducing data from interferometry or occultations, the authors quote either the values obtained when assuming the radiating disc to be uniform (UD; diameter $\left.\Phi_{\mathrm{UD}}\right)$ or limb-darkened (LD; diameter $\left.\Phi_{\mathrm{LD}}\right)$. The extreme case is that of a disc fully-darkened at the limb (FDD; diameter $\left.\Phi_{\mathrm{FDD}}\right)$, the intensity dropping to zero at the limb. The older occultation observations can be found in Ridgway et al. (1977, 1980 and 1982), Walker et al. (1979), Blow et al. (1982), Schmidtke et al. (1986), and references therein. The catalogue from White \& Feiermann (1987) is also useful. Some results have been revisited, such as those of TX Psc and Y Tau (see e.g. Richichi et al. 1995). Part of the observations have been made in the visible but most were in the near infrared, typically through the $H$ and $K$-filters (centered at 1.65 and $2.2 \mu \mathrm{m}$ respectively). Later on, the techniques of the longbaseline interferometry were developed and a wealth of new data became available (Quirrenbach et al. 1994; Dyck et al. 1996b; van Belle et al. 1997 including several carbon Miras). With the recent publications of van Belle et al. (1999, 19 additional stars) and Richichi et al. (1998a \& 1998b, 3 stars), the total number of available angular diameters was increased to 54 for 52 carbon variables of the CV-groups. Unfortunately, no data was available for the HC-stars which are fainter and mostly non-variable. They deserve a specific approach (Sects. 10 and 11).

We have compared data for carbon variables observed at several wavelengths, from distinct methods at different epochs, whenever available (see also van Belle et al. 1999). Dispersion is high enough and appears sometimes larger than quoted by the observers, but no systematic differences could be proved. This is not the case for the coolest oxygen-rich variables, who show much larger diameters in the strong $\mathrm{TiO}$ bands than outside, but the interpretation is difficult (e.g. Jacob et al. 2000 and references therein). No such strong effect seems to be present in the $\mathrm{CN}$ and $\mathrm{C}_{2}$ bands, in the visible and near infrared, not so for the strong $3.1 \mu \mathrm{m}$ band of $\mathrm{HCN}$ and $\mathrm{C}_{2} \mathrm{H}_{2}$ since it can be partially circumstellar in origin. Aoki et al. (1999) found HCN in emission in TX Psc and V CrB near $14 \mu \mathrm{m}$, as did Cernicharo (1998) in IRC+10216. We admit that, in carbon stars spectra, data beyond $2.5 \mu \mathrm{m}$ may be contaminated by circumstellar emission and should not be considered as "photospheric" in origin. We also note that the error bars are frequently as large as the difference between diameters for a uniform disc and a limb-darkened one, for a given carbon star. The data is compiled in Table 2 together with our photometric solutions and integrated fluxes.

\subsection{Direct effective temperatures}

For stars with negligible atmospheric extension, i.e. modeled making use of plane-parallel geometry, the effect of limb-darkening on the estimated angular diameter amounts to only a few percent, especially in the near infrared. When the observational errors and uncertainties about the true brightness law are taken into account, it is not necessary to consider such a small (and questionable) correction. For those stars, we thus adopt

$\Phi \simeq \Phi_{\mathrm{UD}}$

The relative extensions $\Delta r / R$ of model atmospheres of carbon-rich stars with spherical symmetry increase with decreasing $\log g$ at constant effective temperature $\left(T_{\text {eff }}\right)$, and $\mathrm{C} / \mathrm{O}$ ratio: this is the main predicted effect (Jorgensen et al. 1996; Fig. 3, p. 266). Setting $\log g$, (say at -0.5 , see Knapik et al. 2000), the relative extension increases with increasing $T_{\text {eff }}$ at constant $g$, and with increasing $\mathrm{C} / \mathrm{O}$ (and also metallicity) at constant $T_{\text {eff }}$. Carbon stars with static atmospheres should have relative extensions ranging from about $10 \%$ to $25 \%$, (Jorgensen et al. 1996). Pulsations certainly help leading to still larger relative extensions, a phenomenon which is not included in their static models. Van Belle et al. (1996) have compared visibility curves at $2.2 \mu \mathrm{m}$ for a sample of oxygen-rich Miras to those computed from the model atmospheres of Scholz \& Takeda (1987). They deduced that

$\Phi_{\mathrm{LD}} / \Phi_{\mathrm{UD}} \simeq 1.23$.

Following van Belle et al. (1996), we favor the use of a "Rosseland diameter"

$\Phi_{\mathrm{R}}=(\Phi)_{\tau_{\mathrm{R}}=1}$

that is the diameter for which the mean Rosseland optical depth (as evaluated on the whole spectral range) amounts to unity. The models of Scholz \& Takeda (1987) yield

$\Phi_{\mathrm{LD}} / \Phi_{\mathrm{R}} \simeq 1.17$

which results in

$\Phi_{\mathrm{R}} / \Phi_{\mathrm{UD}} \simeq 1.05$,

a conclusion which is consistent with theoretical expectations (Wilson 1986). According to Dyck et al. (1996b), the obtained averaged ratio is

$\left\langle\Phi_{\mathrm{R}} / \Phi_{\mathrm{UD}}\right\rangle \simeq 1.022$ 
Table 2. The observed angular diameters of carbon stars from published data and error bars (Col. 8). Deduced "direct" effective temperatures (Col. 9) and remarks (Miras: M, and phases for photometry and, between parentheses, for diameters; Col. 10) are also given. The $m_{\mathrm{b} 2}$-value for $\mathrm{C} 1653=\mathrm{BM} \mathrm{Gem}$, a carbon star with the silicate signature in emission at $10 \mu \mathrm{m}$, was obtained without the infrared excess (see Sect. 13). Star names and photometric solutions are quoted in columns 1 to 6 with the same meanings as in Table 1; (a) contribution from circumstellar extinction. The derived apparent bolometric magnitude can be found in Col. 7

\begin{tabular}{|c|c|c|c|c|c|c|c|c|c|}
\hline $\mathrm{C}$ & name & $\mathrm{CV}$ & $E(B-V)$ & $\mathrm{Cl}$ & $F_{\mathrm{sp}}$ & $m_{\text {bol }}$ & $\Phi$ & $T_{\text {effd }}$ & Remarks \\
\hline 36 & VX And & 6 & 0.00 & II & $4.8910^{-14}$ & 4.27 & $6.6 \pm 0.6$ & $2410 \pm 115$ & \\
\hline 65 & AQ And & 5 & 0.00 & I & $3.1610^{-14}$ & 4.75 & $4.0 \pm 0.8$ & $2775 \pm 280$ & \\
\hline 198 & $\mathrm{Z} \mathrm{Psc}_{\mathrm{sc}}$ & 2 & 0.03 & I & $7.3510^{-14}$ & 3.83 & $4.8 \pm 0.7$ & $3130 \pm 230$ & \\
\hline 643 & SY Per & 5 & 0.43 & I & $2.6910^{-14}$ & 4.92 & $3.4 \pm 0.8$ & $2890 \pm 340$ & \\
\hline 714 & V718 Tau & 7 & 0.75 & II & $6.0110^{-15}$ & 6.55 & $4.2 \pm ? ? ?$ & $1790 \pm ? ? ?$ & M 0.54 \\
\hline 797 & V346 Aur & $\mathrm{SCV}$ & 0.27 & I & $3.1810^{-14}$ & 4.74 & $3.33 \pm 0.07$ & $3045 \pm 50$ & \\
\hline 833 & R Lep & 6 & 0.02 & I & $1.9510^{-13}$ & 2.77 & $11.5 \pm 0.64$ & $2500 \pm 80$ & M $0.08(0.99)$ \\
\hline 853 & W Ori & 5 & 0.00 & I & $1.7810^{-13}$ & 2.87 & $9.7 \pm 0.6$ & $2745 \pm 90$ & \\
\hline 941 & S Aur & 7 & 0.28 & II & $2.8910^{-14}$ & 4.84 & $8.9 \pm 0.6$ & $1845 \pm 70$ & \\
\hline 988 & RT Ori & 4 & 0.07 & I & $2.9310^{-14}$ & 4.83 & $4.4 \pm 0.9$ & $2595 \pm 270$ & \\
\hline 1006 & IRC +20115 & 6 & 0.13 & I & $7.4710^{-15}$ & 6.31 & $2.49 \pm 0.12$ & $2450 \pm 70$ & \\
\hline 1038 & TU Tau & 3 & 0.38 & I & $3.4910^{-14}$ & 4.64 & $3.82 \pm 0.08$ & $2911 \pm 50$ & \\
\hline 1042 & Y Tau & 4 & 0.19 & I & $1.1710^{-13}$ & 3.33 & $8.18 \pm 0.50$ & $2690 \pm 90$ & \\
\hline 1264 & BL Ori & 2 & 0.0 & I & $7.9810^{-14}$ & 3.74 & $3.56 \pm 0.08$ & $3707 \pm 60$ & \\
\hline 1269 & AB Gem & 6 & 0.16 & I & $1.2310^{-14}$ & 5.77 & $4.06 \pm 0.09$ & $2180 \pm 40$ & \\
\hline 1300 & RV Aur & 3 & 0.12 & I & $1.0210^{-14}$ & 5.97 & $1.97 \pm 0.08$ & $2981 \pm 48$ & \\
\hline 1309 & CR Gem & 3 & 0.67 & I & $5.1310^{-14}$ & 4.22 & $3.79 \pm 0.09$ & $3217 \pm 56$ & \\
\hline 1316 & UU Aur & 4 & 0.09 & I & $2.9610^{-13}$ & 2.32 & $12.1 \pm 0.2$ & $2790 \pm 40$ & \\
\hline 1355 & VW Gem & 2 & 0.05 & I & $1.3910^{-14}$ & 5.46 & $2.13 \pm 0.04$ & $3096 \pm 48$ & $\operatorname{int}(?)$ \\
\hline 1489 & RV Mon & 3 & 0.0 & I & $4.2410^{-14}$ & 4.43 & $3.35 \pm 0.07$ & $3262 \pm 53$ & \\
\hline 1595 & VX Gem & 3 & 0.10 & I & $9.7010^{-15}$ & 6.03 & $2.07 \pm 0.10$ & $2871 \pm 78$ & \\
\hline 1653 & BM Gem & 1 & 0.13 & I & $1.0210^{-14}$ & 5.97 & $2.16 \pm 0.04$ & $2845 \pm 60$ & $m_{\mathrm{b} 2}=6.17$ \\
\hline 2378 & $\mathrm{X}$ Cnc & 5 & 0.00 & I & $1.0010^{-13}$ & 3.49 & $7.76 \pm 0.7$ & $2660 \pm 120$ & \\
\hline 2384 & $\mathrm{~T}$ Cnc & 6 & 0.00 & I & $4.8210^{-14}$ & 4.29 & $7.1 \pm 0.1$ & $2315 \pm 30$ & 0.1 \\
\hline 2384 & T Cnc & 6 & $0.36^{a}$ & I & $5.8410^{-14}$ & 4.08 & $7.1 \pm 0.1$ & $2370 \pm 35$ & 0.7 \\
\hline 3236 & SS Vir & 6 & 0.00 & I & $8.3410^{-14}$ & 3.69 & $8.71 \pm 0.49$ & $2400 \pm 75$ & 0.0 \\
\hline 3283 & Y CVn & 5 & 0.00 & II & $2.6610^{-13}$ & 2.43 & $11.6 \pm 0.3$ & $2775 \pm 50$ & \\
\hline 3652 & $\mathrm{~V}$ CrB & 7 & 0.00 & II & $2.9210^{-14}$ & 4.83 & $7.26 \pm 0.23$ & $2020 \pm 40$ & M $0.15(0.08)$ \\
\hline 3837 & TW Oph & 6 & 0.37 & I & $1.1410^{-13}$ & 3.36 & $9.99 \pm 0.5$ & $2420 \pm 70$ & \\
\hline 3875 & SZ Sgr & 1 & 0.50 & I & $2.6310^{-14}$ & 4.94 & $3.18 \pm 0.16$ & $2970 \pm 80$ & \\
\hline 3933 & V4378 Sgr & 1 & 1.09 & I & $1.8610^{-14}$ & 5.32 & $1.58 \pm 0.88$ & $3870 \pm 1080$ & \\
\hline 4089 & HK Lyr & 5 & 0.05 & I & $2.7610^{-14}$ & 4.89 & $3.52 \pm 0.06$ & $2858 \pm 43$ & \\
\hline 4111 & DR Ser & 5 & 0.36 & I & $2.1510^{-14}$ & 5.16 & $4.11 \pm 0.14$ & $2485 \pm 55$ & \\
\hline 4164 & V Aql & 6 & 0.15 & I & $1.4410^{-13}$ & 3.10 & $10.1 \pm 0.7$ & $2550 \pm 380$ & \\
\hline 4241 & $\mathrm{U}$ Lyr & 5 & 0.13 & I & $1.6810^{-14}$ & 5.43 & $3.58 \pm 0.09$ & $2504 \pm 44$ & $0.06(?)$ \\
\hline 4333 & AQ Sgr & 4 & 0.11 & I & $7.3110^{-14}$ & 3.83 & $6.0 \pm 0.5$ & $2795 \pm 120$ & \\
\hline 4415 & TT Cyg & 4 & 0.03 & I & $2.5210^{-14}$ & 4.99 & $3.23 \pm 0.07$ & $2918 \pm 48$ & \\
\hline 4758 & RS Cyg & 2 & 0.35 & I & $6.2910^{-14}$ & 4.00 & $4.3 \pm 0.8$ & $3180 \pm 300$ & \\
\hline 4774 & RT Cap & 6 & 0.00 & I & $7.5710^{-14}$ & 3.80 & $7.72 \pm 0.16$ & $2485 \pm 40$ & \\
\hline 4817 & U Cyg & 6 & 0.81 & I & $5.4210^{-14}$ & 4.16 & $6.96 \pm 0.50$ & $2410 \pm 90$ & M $0.66(0.67)$ \\
\hline 4939 & V Cyg & 7 & 0.41 & II & $1.0810^{-13}$ & 3.41 & $14.20 \pm 0.77$ & $2000 \pm 60$ & M $0.20(0.25)$ \\
\hline 5265 & YY Cyg & 4 & 0.28 & I & $1.3110^{-14}$ & 5.70 & $2.28 \pm 0.65$ & $2949 \pm 422$ & \\
\hline 5358 & V1426 Cyg & 7 & $1.18^{a}$ & II & $5.8510^{-14}$ & 4.08 & $10.8 \pm 0.4$ & $1970 \pm 45$ & M $0.85(0.2 ?)$ \\
\hline 5358 & V1426 Cyg & 7 & 0.41 & II & $5.1510^{-14}$ & 4.21 & $10.8 \pm 0.4$ & $1910 \pm 40$ & M $0.15(0.2 ?)$ \\
\hline 5406 & S Cep & 6 & 0.21 & I & $1.5110^{-13}$ & 3.05 & $13.67 \pm 0.76$ & $2220 \pm 70$ & M $0.05(0.22)$ \\
\hline 5418 & V460 Cyg & 2 & 0.11 & I & $1.3210^{-13}$ & 3.19 & $6.3 \pm 0.6$ & $3160 \pm 160$ & \\
\hline 5425 & RV Cyg & 5 & 0.39 & I & $1.0310^{-13}$ & 3.46 & $7.6 \pm 0.5$ & $2705 \pm 95$ & $0.44(0.56)$ \\
\hline 5494 & LW Cyg & 5 & 0.21 & I & $3.1610^{-14}$ & 4.75 & $4.00 \pm 0.07$ & $2773 \pm 42$ & \\
\hline 5496 & RX Peg & 3 & 0.06 & I & $1.9510^{-14}$ & 5.27 & $2.89 \pm 0.14$ & $2892 \pm 79$ & \\
\hline 5570 & RZ Peg & 5 & 0.27 & I & $2.0410^{-14}$ & 5.22 & $3.04 \pm 0.02$ & $2852 \pm 37$ & $0.22(?)$ \\
\hline 5791 & VY And & 5 & 0.27 & I & $8.5610^{-15}$ & 6.16 & $2.40 \pm 0.02$ & $2584 \pm 34$ & \\
\hline 5928 & TX Psc & 2 & 0.03 & I & $2.7210^{-13}$ & 2.41 & $9.31 \pm 0.75$ & $3115 \pm 130$ & \\
\hline 5976 & WZ Cas & 2 & 0.34 & II & $9.1910^{-14}$ & 3.55 & $5.8 \pm 0.7$ & $3010 \pm 185$ & $0.6(0.0 ?)$ \\
\hline 5987 & SU And & 3 & 0.00 & I & $1.5810^{-14}$ & 5.50 & $2.32 \pm 0.14$ & $3063 \pm 400$ & \\
\hline
\end{tabular}




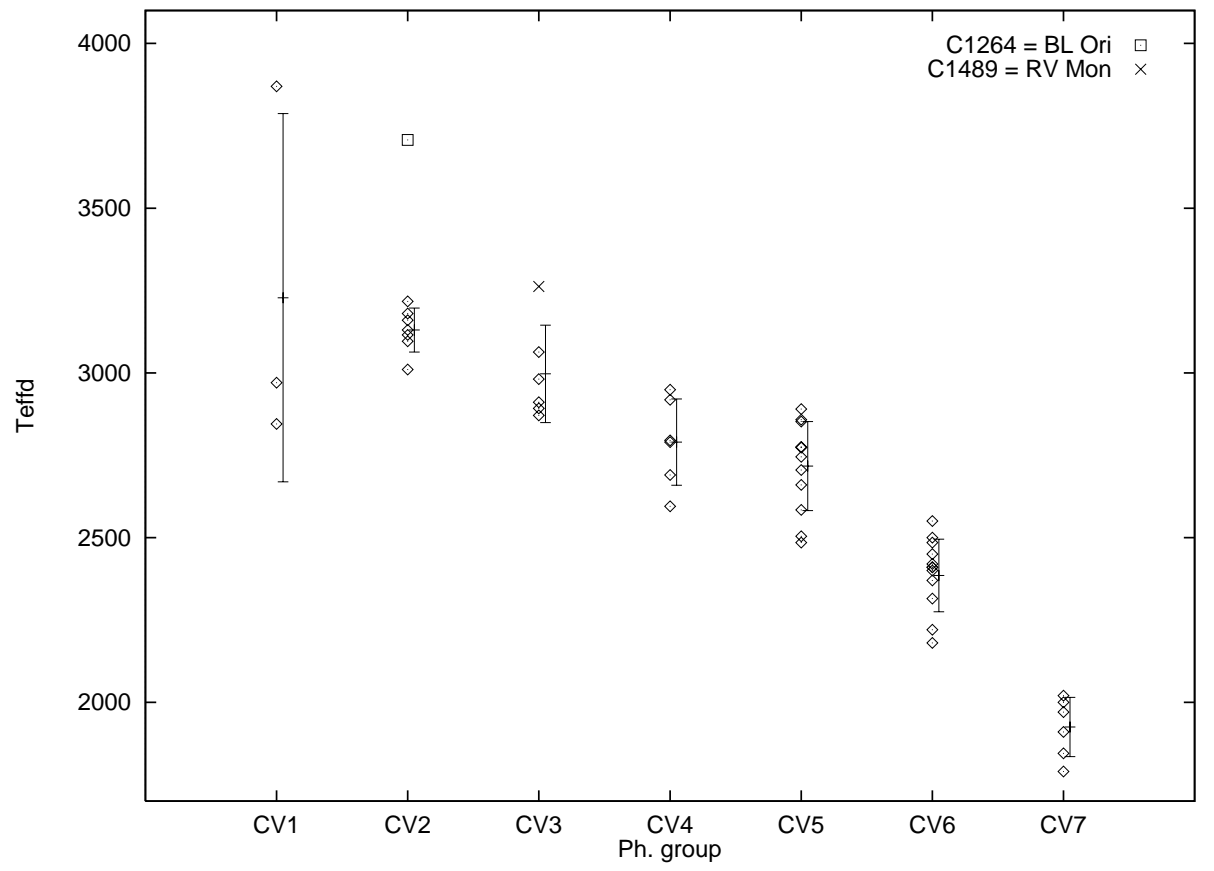

Fig. 3. The effective temperatures from 51 observed angular diameters (see Sect. 5) as a function of our photometric CV-group. Data is taken from Table 2 (the 52 nd star C797 =V 346 Aur, classified SCV, is not shown). Mean values are also shown for the seven CV-groups together with dispersions (see Table 3). A regular decrease in $T_{\text {eff }}$ with increasing group number is observed, except at CV4-CV5 where a shoulder is noticed. Two stars are labelled which were not included in the means. The CV1 mean is quite uncertain since obtained from only 3 highly dispersed values

Table 3. Mean direct effective temperatures and dispersions derived from 51 values from Table 2 , for the seven CV photometric groups. The only value available for the SCV-group (C797 = V346 Aur) is also quoted

\begin{tabular}{ccccccccc}
\hline Group & CV1 & CV2 & CV3 & CV4 & CV5 & CV6 & CV7 & SCV \\
\hline$n$ & 3 & 7 & 5 & 6 & 12 & 12 & 6 & 1 \\
$\left\langle T_{\text {effd }}\right\rangle \pm \epsilon_{T_{\text {effd }}}$ & $3230 \pm 560$ & $3130 \pm 70$ & $2940 \pm 80$ & $2790 \pm 130$ & $2720 \pm 135$ & $2385 \pm 110$ & $1925 \pm 90$ & 2880 \\
\hline
\end{tabular}

for carbon-rich and oxygen-rich variables. Observational errors and uncertainties about the true brightness distributions taken into account, we adopt

$\Phi \simeq \Phi_{\mathrm{R}} \simeq \Phi_{\mathrm{UD}}$

These $\Phi_{\text {UD }}$ diameters are quoted in Table 2 . They were used to estimate "direct" effective temperatures from

$F=F_{\mathrm{sp}} \simeq\left(\Phi^{2} / 4\right) \sigma T_{\mathrm{effd}}^{4}$

where $\sigma$ is Stefan's constant. Following Dyck et al. (1996), we have calculated the associated errors from

$\epsilon_{T_{\text {effd }}} / T_{\text {effd }} \simeq\left[\left(\epsilon_{F} / 4 F\right)^{2}+\left(\epsilon_{\Phi} / 2 \Phi\right)^{2}\right]^{1 / 2}$.

As a typical value, we have adopted $\epsilon_{F} / F \simeq 0.05$. The relation derived from Eq. (15) is

$T_{\text {effd }} \simeq 1.31610^{7} F^{1 / 4} \Phi^{-1 / 2}$

where $F$ is in $\mathrm{W} \mathrm{cm}^{-2}$ and $\Phi$ in mas. The comparison of the present data with predictions of available model atmospheres leads to a good consistency (see Sects. 9 and 10). On the contrary, the adoption of

$\Phi \simeq \Phi_{\mathrm{LD}} \simeq 1.23 \Phi_{\mathrm{UD}}$ would result in temperatures lower by about 250 to $400 \mathrm{~K}$, close to the mean color temperatures (Sect. 15). A marked discrepancy is noted between dereddened SEDs from observations and those of such cooler model atmospheres.

\section{Direct effective temperatures and photometric groups}

Considering the regular evolution of color indices along the $\mathrm{HC} 0$ to HC5 photometric sequence, followed by the CV1 to CV7 sequence, a tight relation between effective temperatures and photometric groups is expected (Fig. 3; data from Table 2). Mean values and dispersions are also shown for the $7 \mathrm{CV}$-groups and quoted in Table 3. Two stars $(\mathrm{C} 1264=\mathrm{BL}$ Ori classified CV2 and C1489 = RV Mon classified CV3) were not included, since they significantly depart from the loci of the other stars. The mean of the three dispersed values for CV1 is indicative only.

A good correlation is observed between the effective temperatures and our CV classification in photometric groups. A shoulder is observed at CV4-CV5 which we ascribe to a non-uniformity of the CV-scale. The frequency distribution over the $\mathrm{CV}$-groups shows a minimum at 
Table 4. Photometric data for the 54 SEDs of Table 2. The $V_{0}$-magnitudes and selected dereddened color indices are adapted from the photometric solutions adopted in Papers I and III. The indices are respectively $I_{1}=(V-[1.08])_{0}, I_{2}=(V-K)_{0}$, $I_{3}=([1.08]-K)_{0}, I_{4}=(J-K)_{0}$, and $I_{5}=(H-K)_{0}$

\begin{tabular}{|c|c|c|c|c|c|c|c|c|c|c|c|c|c|c|c|}
\hline $\bar{C}$ & $\overline{V_{0}}$ & $I_{1}$ & $I_{2}$ & $I_{3}$ & $I_{4}$ & $I_{5}$ & $\log T_{\text {effd }}$ & $\bar{C}$ & $\overline{V_{0}}$ & $I_{1}$ & $I_{2}$ & $I_{3}$ & $I_{4}$ & $I_{5}$ & $\log T_{\text {effd }}$ \\
\hline 36 & 7.48 & 4.15 & 6.58 & 2.22 & 1.95 & 0.78 & 3.382 & 65 & 7.81 & 4.25 & 6.23 & 1.98 & 1.76 & 0.62 & 3.443 \\
\hline 198 & 5.99 & 3.43 & 5.20 & 1.77 & 1.50 & 0.42 & 3.496 & 643 & 8.00 & 4.21 & 6.18 & 1.97 & 1.64 & 0.60 & 3.461 \\
\hline 714 & 11.99 & 5.02 & 8.30 & 3.28 & 2.83 & 1.31 & 3.253 & 797 & 7.92 & 4.47 & 6.39 & 1.92 & 1.58 & 0.46 & 3.484 \\
\hline 833 & 6.44 & 4.37 & 6.81 & 2.44 & 2.11 & 0.79 & 3.398 & 853 & 6.06 & 4.31 & 6.36 & 2.05 & 1.77 & 0.67 & 3.439 \\
\hline 941 & 9.86 & 5.01 & 8.21 & 3.20 & 2.74 & 1.24 & 3.266 & 988 & 7.65 & 4.05 & 5.91 & 1.86 & 1.60 & 0.48 & 3.414 \\
\hline 1006 & 9.83 & 4.43 & 6.85 & 2.42 & 2.15 & 0.85 & 3.389 & 1038 & 7.07 & 3.96 & 5.81 & 1.85 & 1.61 & 0.62 & 3.464 \\
\hline 1042 & 6.31 & 4.19 & 6.05 & 1.86 & 1.71 & 0.52 & 3.430 & 1264 & 6.20 & 3.74 & 5.46 & 1.72 & 1.41 & 0.40 & 3.569 \\
\hline 1269 & 9.20 & 4.43 & 6.65 & 2.22 & 1.91 & 0.73 & 3.338 & 1300 & 8.57 & 3.80 & 5.68 & 1.88 & 1.61 & 0.55 & 3.474 \\
\hline 1309 & 6.72 & 3.67 & 5.53 & 1.86 & 1.50 & 0.46 & 3.507 & 1316 & 5.29 & 4.12 & 6.01 & 1.89 & 1.59 & 0.49 & 3.446 \\
\hline 1355 & 8.15 & 3.75 & 5.50 & 1.75 & 1.44 & 0.44 & 3.491 & 1489 & 5.29 & 4.12 & 6.01 & 1.89 & 1.59 & 0.49 & 3.446 \\
\hline 1595 & 8.65 & 3.84 & 5.72 & 1.88 & 1.59 & 0.53 & 3.458 & 1653 & 8.03 & 3.41 & 5.11 & 1.70 & 1.47 & 0.50 & 3.454 \\
\hline 2378 & 6.55 & 4.12 & 6.26 & 2.14 & 1.85 & 0.61 & 3.425 & 2384 & 7.89 & 4.62 & 6.83 & 2.21 & 1.96 & 0.73 & 3.365 \\
\hline 2384 & 7.52 & 4.32 & 6.64 & 2.32 & 1.83 & 0.70 & 3.375 & 3236 & 6.85 & 4.11 & 6.36 & 2.25 & 1.88 & 0.72 & 3.380 \\
\hline 3283 & 5.60 & 4.40 & 6.43 & 2.03 & 1.74 & 0.62 & 3.443 & 3652 & 9.48 & 5.04 & 7.93 & 2.89 & 2.37 & 0.91 & 3.305 \\
\hline 3837 & 6.71 & 4.38 & 6.55 & 2.17 & 1.83 & 0.96 & 3.384 & 3875 & 7.09 & 3.49 & 5.16 & 1.67 & 1.36 & 0.43 & 3.473 \\
\hline 3933 & 7.33 & 3.36 & 4.99 & 1.63 & 1.38 & 0.42 & 3.588: & 4089 & 8.16 & 4.46 & 6.45 & 1.99 & 1.86 & 0.63 & 3.456 \\
\hline 4111 & 8.22 & 4.14 & 6.30 & 2.16 & 1.95 & 0.68 & 3.395 & 4164 & 8.71 & 4.28 & 6.50 & 2.22 & 1.90 & 0.82 & 3.399 \\
\hline 4241 & 6.58 & 4.48 & 6.71 & 2.23 & 1.70 & 0.71 & 3.407 & 4333 & 6.68 & 4.04 & 5.95 & 1.91 & 1.69 & 0.55 & 3.446 \\
\hline 4415 & 7.89 & 4.24 & 5.98 & 1.74 & 1.65 & 0.60 & 3.465 & 4758 & 6.44 & 3.75 & 5.45 & 1.70 & 1.38 & 0.42 & 3.502 \\
\hline 4774 & 7.29 & 4.49 & 6.73 & 2.24 & 1.82 & 0.66 & 3.395 & 4817 & 7.60 & 4.38 & 6.54 & 2.16 & 1.83 & 0.61 & 3.382 \\
\hline 4939 & 8.72 & 4.97 & 8.46 & 3.31 & 2.88 & 1.42 & 3.301 & 5265 & 8.51 & 4.02 & - & - & - & - & 3.470 \\
\hline 5358 & 9.34 & 5.57 & 8.44 & 2.99 & 2.57 & 1.07 & 3.294 & 5358 & 9.52 & 5.00 & 8.31 & 3.31 & 2.93 & 1.18 & 3.281 \\
\hline 5406 & 6.76 & 4.40 & 7.03 & 2.63 & 2.24 & 0.86 & 3.346 & 5418 & 5.73 & 3.73 & 5.53 & 1.80 & 1.49 & 0.47 & 3.500 \\
\hline 5425 & 6.44 & 4.13 & 6.17 & 2.04 & 1.68 & 0.63 & 3.432 & 5494 & 8.05 & 4.40 & 6.53 & 2.13 & 1.85 & 0.69 & 3.443 \\
\hline 5496 & 7.89 & 3.85 & 5.68 & 1.83 & - & - & 3.461 & 5570 & 8.38 & 4.27 & - & - & - & - & 3.455 \\
\hline 5791 & 9.04 & 4.26 & 6.09 & 1.83 & 1.79 & 0.59 & 3.412 & 5928 & 4.84 & 3.76 & 5.47 & 1.71 & 1.48 & 0.43 & 3.493 \\
\hline 5976 & 6.00 & 3.79 & 5.54 & 1.75 & 1.42 & 0.39 & 3.479 & 5987 & 8.21 & 3.97 & 5.77 & 1.80 & 1.62 & 0.48 & 3.486 \\
\hline
\end{tabular}

CV4. If the CV4 and CV5 stars were gathered in a single group, the latter would be the most populated one, i.e. it would represent a maximum in the distribution. Thus we consider the shoulder of Fig. 3 as due to the nonuniformity of the classification which is tighter in effective temperatures at CV4-CV5. A further improvement may well be continuous parameterization as opposed to the discrete groups we adopted. The main parameter of our classification into 13 photometric ( $H C$ and $C V$ ) groups, as elaborated in Papers I, II and III, is effective temperature. This classification may play the role of spectral types for oxygen-rich stars in the Harvard classification. The discussion of other parameters such as gravity and mass in an atmosphere with spherical geometry, or chemical abundances, is deferred to a later investigation (Knapik et al. 2000, see however Sects. 17 and 18).

\section{Calibrations of the relations between color indices and effective temperatures}

We calibrated five dereddened color indices in terms of the direct effective temperatures of Table 2. The selected indices were $(V-[1.08])_{0},([1.08]-K)_{0},(V-K)_{0}$, $(J-K)_{0}$, and $(H-K)_{0}$. The color indices of the $(R-I)_{0}$ category, with $R$ 's and $I$ 's in various systems, should not be considered since they vary little along the CVsequence (i.e. with effective temperature: Sect. 6). Other combinations can be used e.g. $(V-[0.78])_{0}$. Indices like $(K-L)_{0}$ or $(K-[12])_{0}$ were not selected for various reasons $(L$-magnitudes more dispersed, SiC-excesses centered between 11 and $11.5 \mu \mathrm{m})$. The values of $V_{0}$ and those of the five selected indices are quoted in Table 4.

As an illustration, the relation between $\log T_{\text {effd }}$ and $(J-K)_{0}$ is shown in Fig. 4. Apart from C1269= AB Gem (and C3933 = V4378 Sgr which lies outside the displayed frame), the stars populate a relatively well-defined strip, with $\Delta \log T_{\text {effd }} \simeq 0.1$ (i.e. \pm 0.05$)$ and $\Delta(J-K)_{0} \simeq$ $0.25-0.30$ (i.e. $\pm 0.12-0.15$ ). A marked elbow is observed at $(J-K)_{0} \simeq 2.0-2.2$ i.e. $T_{\text {eff }} \simeq 2250 \mathrm{~K}$, for the coolest CV6-stars. Taking into account the small number of the available points, we adjust two linear fits with a junction at $(J-K)_{0}=2.1$, making use of the least-squares method. The relations with the other indices also display such a bend, with the exception of $(V-[1.08])_{0}$.

The authors already noticed in Paper III a gap in the photometric indices when passing from CV6 to CV7. The proposed interpretation was a substantial change in the opacities coupled with the low effective temperatures, i.e. 1900-2500 K. Increasing opacities of molecules and grains are coupled with emission from dust substantially contaminating the $K$-bandpass and slightly the $H$ bandpass, while the $J$-one is almost free of excesses. We assume that the observers have been able to disentangle the 


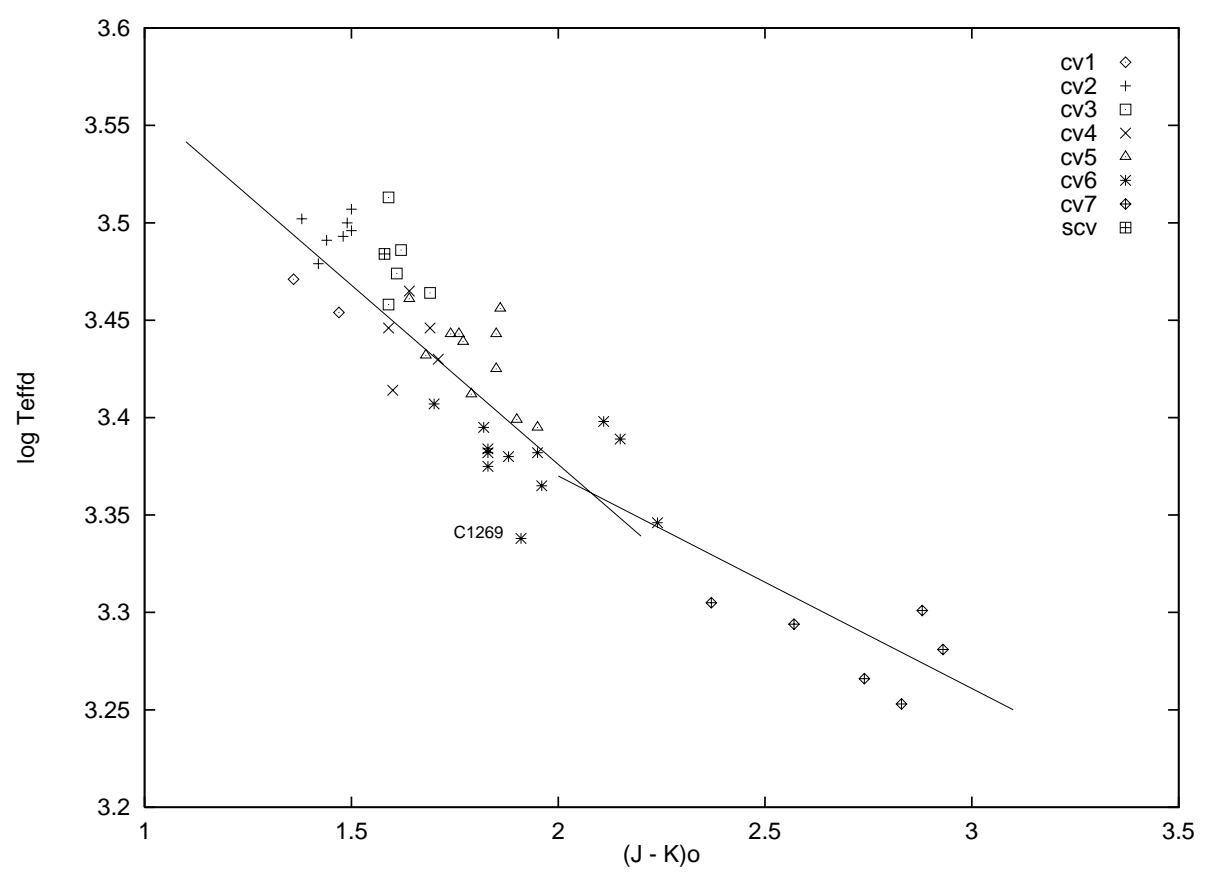

Fig. 4. The relation between $\log T_{\text {effd }}$ from Table 2 , and $(J-K)_{0}$ together with the two regression lines (19) adopted for $(J-K)_{0} \leq 2.1$ and $(J-K)_{0} \geq 2.1$ respectively. The star C1269 = AB Gem was not included in the fits

circumstellar contributions to their occultation or interferometric data. If the angular diameters of their coolest stars were overestimated due to failures in data reduction, the effective temperatures of Table 4 would then be underestimated, and curvatures in the relations should be still more pronounced.

The linear fits obtained for the five photometric indices $C I_{j}$ are written as $(j=1$ to 5$)$

$\log T_{\text {effd }}=\mu_{1} C I_{j, 0}+\mu_{2}$

and the coefficients as deduced from the least-squares method are given in Table 5. The transitions, as quoted in Col. 6, do occur in the CV6 domain, except for $(H-K)_{0}$ whose elbow lies in the CV7. This is consistent with the interpretation we proposed above, i.e. a $H$-bandpass less contaminated than the $K$-one. Despite low $\left|\mu_{1}\right|$ values, the correlation coefficient ranges from 0.62 to 0.90 which is satisfactory. The calibrations (19) of Table 5 are considered as provisional, especially at high temperatures. We recommend to calculate a mean value by combining them together with presumably equal weights, which necessitates the knowledge of five magnitudes. Improved calibrations are proposed in Table 11 which can be used individually (see Sect. 12).

\section{The relations between measured angular diameters and photometric $\langle k\rangle^{\mathbf{1 / 2}}$-factors}

We have introduced in Paper I a photometric coefficient, the $\langle k\rangle$-factor, which was used throughout our analyses. Its practical definition can be found in Sect. 2.4 of Paper III, and it was suggested that it could be a squared angular diameter on a relative scale. Here we intend to
Table 5. Calibration of direct effective temperatures against five color indices (Eq. (19)). Except for $(V-[1.08])_{0}$, two separate linear fits are given with validity ranges in Col. 6 . The number of used points is $\mathrm{n}$ and the correlation coefficient is $\rho^{2}$

\begin{tabular}{cccccc}
\hline$C I_{j, 0}$ & $\mathrm{n}$ & $\mu_{1} \pm \epsilon_{\mu_{1}}$ & $\mu_{2} \pm \epsilon_{\mu_{2}}$ & $\rho^{2}$ & $\Delta C I_{j, 0}$ \\
\hline$[V-[1.08]]_{0}$ & 35 & $-0.140 \pm 0.011$ & $4.01 \pm 0.03$ & 0.82 & $3.3-5.6$ \\
{$[V-K]_{0}$} & 28 & $-0.079 \pm 0.008$ & $3.91 \pm 0.02$ & 0.78 & $\leq 7.0$ \\
{$[V-K]_{0}$} & 14 & $-0.061 \pm 0.008$ & $3.79 \pm 0.02$ & 0.81 & $\geq 7.0$ \\
{$[[1.08]-K]_{0}$} & 26 & $-0.164 \pm 0.019$ & $3.76 \pm 0.02$ & 0.76 & $\leq 2.3$ \\
{$[[1.08]-K]_{0}$} & 19 & $-0.115 \pm 0.009$ & $3.65 \pm 0.02$ & 0.90 & $\geq 2.3$ \\
{$[J-K]_{0}$} & 27 & $-0.184 \pm 0.024$ & $3.74 \pm 0.03$ & 0.70 & $\leq 2.1$ \\
{$[J-K]_{0}$} & 12 & $-0.109 \pm 0.022$ & $3.59 \pm 0.03$ & 0.72 & $\geq 2.1$ \\
{$[H-K]_{0}$} & 26 & $-0.287 \pm 0.040$ & $3.60 \pm 0.03$ & 0.68 & $\leq 0.86$ \\
{$[H-K]_{0}$} & 13 & $-0.169 \pm 0.040$ & $3.50 \pm 0.03$ & 0.62 & $\geq 0.86$ \\
\hline
\end{tabular}

check this hypothesis by comparing the observed angular diameters, as compiled in Sect. 5 and Table 2, to the values of $\langle k\rangle^{1 / 2}$ we obtained for those stars. If confirmed, this expected relation would both widen and strengthen the meaning of our photometric analyses. In addition, it would allow a calibration of the $\langle k\rangle$-factors.

We emphasize here that the latter quantity already showed a correlation with true parallaxes deduced from the HIPPARCOS data, that is expected for stars populating a given range in linear diameters (see Fig. 3 of Knapik et al. 1998). The $k$-factor is the ratio of the dereddened net flux of a given star at a selected wavelength, to the corresponding net flux of a reference star with magnitude $[1.08]_{0}=0$ at $\lambda=1.08 \mu \mathrm{m}$. Ideally, both stars should have the same parameters for model atmospheres, and the same effective temperature. Taking advantage of the good correlation of the classification into photometric groups with the directly deduced effective temperatures (Sect. 6), 


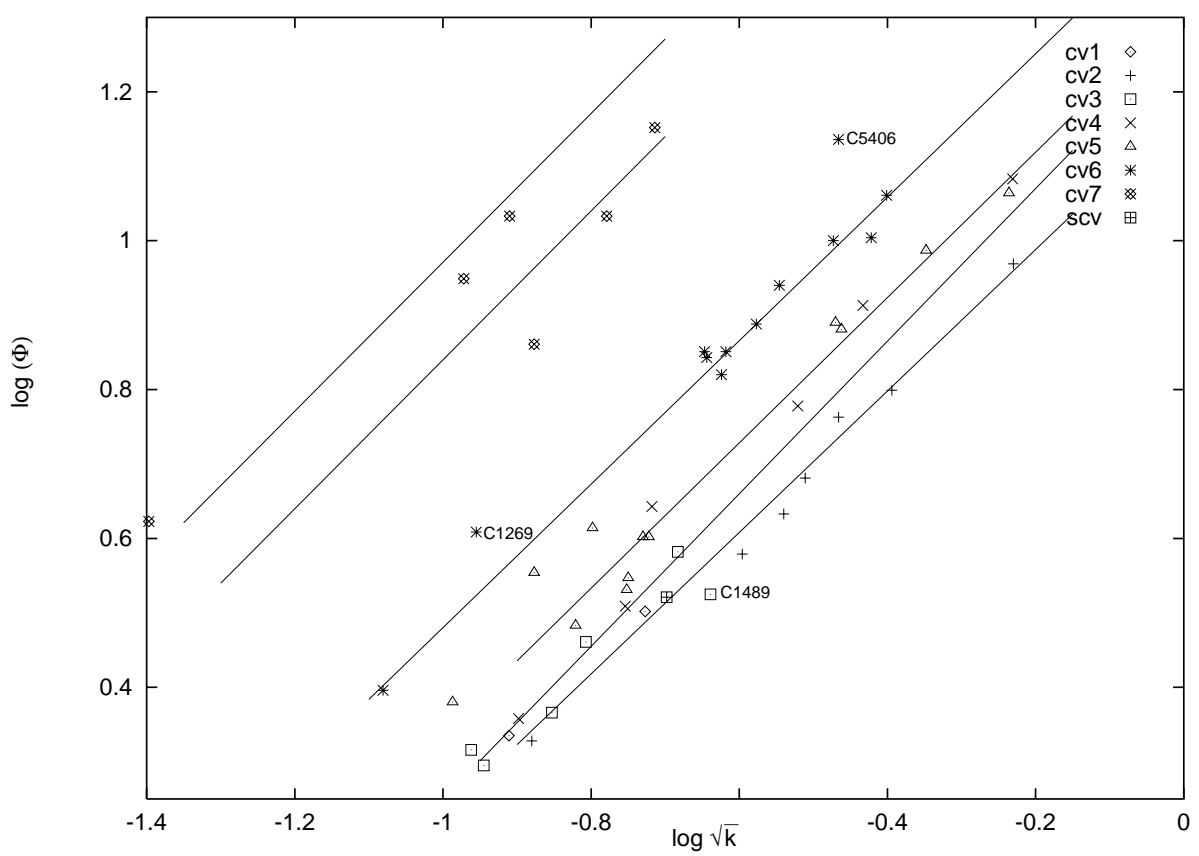

Fig. 5. The relations of $\log \Phi$ vs. $\log \langle k\rangle^{1 / 2}$ for various photometric groups or associations of groups. The six linear fits like Eqs. (25) and (26) are shown (see also Table 6). The stars C1269, C1489 and C5406 were not included in the fits

we intend to define a reference star per group, as a first step. The net flux for a circular disc may be written as

$F_{\lambda}=\pi[\Phi(\lambda) / 2]^{2} I_{\lambda, 0} \quad \eta(\lambda)=\pi[\Phi(\lambda) / 2]^{2} \tilde{I}_{\lambda}$

where the detailed SED from a model atmosphere is described by $\eta(\lambda)$ for a given central intensity $I_{\lambda, 0}$. Thus $\tilde{I}_{\lambda}$ is the specific intensity of an equivalent Lambertian (i.e. isotropic) source. Replacing this intensity by the Planck function would yield the brightness temperature at the considered wavelength. The balance for either global darkening or brightening, is expressed by $\eta(\lambda)$ which amounts to 1 for a uniform disc. For every star classified in a given photometric group, the dereddened SED is proportional, within errors, to a reference distribution, viz.

$F_{\lambda}=\left\langle k\left(\lambda_{i}\right)\right\rangle_{i=1, n} F_{[1.08]=0}^{\mathrm{G}}\left(\lambda_{i}\right)=\langle k\rangle F_{[1.08]=0}^{\mathrm{G}}(\lambda)$.

For two stars belonging to the same group, we thus expect

$F_{\lambda} / F_{\lambda}{ }^{\prime}=\langle k\rangle /\left\langle k^{\prime}\right\rangle$

to be satisfied. We now refer to the second object as the star with $[1.08]_{0}=0$ and $\left\langle k^{\prime}\right\rangle=1$. Hence

$F_{\lambda}{ }^{\prime}=\pi\left[\Phi_{0}(\lambda) / 2\right]^{2} \tilde{I}_{\lambda}^{\prime}=F_{[1.08]=0}^{\mathrm{G}}(\lambda)$.

We obtain

$\langle k\rangle=F_{\lambda} / F_{\lambda}{ }^{\prime}=\left[\Phi(\lambda) / \Phi_{0}(\lambda)\right]^{2}\left[\tilde{I}_{\lambda} / \tilde{I}_{\lambda}^{\prime}\right]$

and then

$$
\begin{array}{r}
\log \Phi(\lambda)=\log \langle k\rangle^{1 / 2}+\log \Phi_{0}(\lambda) \\
-(1 / 2) \log \left(\tilde{I}_{\lambda} / \tilde{I}_{\lambda}^{\prime}\right) .
\end{array}
$$

Thus, in a diagram of $\log \Phi$ vs. $\log \langle k\rangle^{1 / 2}$, we should have a linear relation with slope unity and $\log \Phi_{0}(\lambda)-$ $(1 / 2) \log \left(\tilde{I}_{\lambda} / \tilde{I}_{\lambda}^{\prime}\right)$ for the intercept. As a fit to the data, we use the relation

$\log \Phi=\alpha \log \langle k\rangle^{1 / 2}+\beta$.

The corresponding diagram for the carbon stars with observed angular diameters is shown as Fig. 5. Once more it is unfortunately limited to the CV-stars, no angular diameter having been observed for the HC-stars. We observe that parallel stripes are populated with slopes close to unity and intercepts increasing with group numbers. However, taking into account the limited number and accuracy of the available diameters, and the influence of variability on coupling with the $\langle k\rangle$-coefficients, we have distinguished six groups or associations of groups (see Table 6). The slopes can be assimilated to unity within the dispersions for the first 4 groups or associations. The correlation coefficient is at least 0.96 . Concerning CV7, we adopted 1 since there are too few points and there is some evidence for an increase of the intercept with phase from maximum $(7 \mathrm{max})$ to minimum $(7 \mathrm{~min})$. There is actually no gap between the different groups except possibly in the CV6-CV7 domain, where the intercept increases markedly. The two rejected CV6-stars, namely C1269 and C5406, are however located appreciably above the CV6 linear fit. More data at known phases is needed before a firm conclusion can be reached about a possible gap. The correlation seen in Fig. 5 and described in Table 6 is quite interesting since the data are independent. Let us assume that the stars of a subset $(j=1, m)$ in a given group 
Table 6. The observed angular diameter as a function of the coefficient $\langle k\rangle$ from photometry (Eqs. (25) and (26)). Three stars (C1269, C1489 and C5406) were rejected. The remaining 49 stars were distributed into 6 groups or associations

\begin{tabular}{ccccc}
\hline CV & $n$ & $\alpha$ & $\beta$ & $\rho^{2}$ \\
\hline $1-2-\mathrm{SCV}$ & 10 & $0.950 \pm 0.050$ & $1.178 \pm 0.023$ & 0.986 \\
3 & 5 & $1.025 \pm 0.124$ & $1.275 \pm 0.028$ & 0.958 \\
$4-5$ & 18 & $0.976 \pm 0.050$ & $1.314 \pm 0.048$ & 0.959 \\
6 & 10 & $0.964 \pm 0.034$ & $1.444 \pm 0.020$ & 0.980 \\
$7 \max$ & 4 & 1 adopted & 1.840 & \\
$7 \min$ & 2 & 1 adopted & 1.971 & \\
\hline
\end{tabular}

$\mathrm{G}$, verify the same relation (26) with $\alpha=1$, within the dispersion. We may adopt, for any $j$,

$\log \Phi_{0}-(1 / 2) \log \left(\tilde{I}_{\lambda}^{j} / \tilde{I}_{\lambda}^{\prime}\right) \simeq \beta$

which can be obtained only if

$\tilde{I}_{\lambda}^{j} \simeq \tilde{I}_{\lambda}^{\prime j^{\prime}} \simeq \tilde{I}_{\lambda}^{\prime}$

for any $j, j^{\prime}=1, m$. Hence

$\beta \simeq \log \Phi_{0}$

There is no documented variation with the central wavelength of the observations, at least in the limited data presently available for carbon stars. Part of them were secured in the visible, typically at 0.6 or $0.7 \mu \mathrm{m}$, and a majority observed in the near infrared at 1.6 or $2.2 \mu \mathrm{m}$. A much larger body of data must be awaited before documenting possible dependences like $\Phi_{0}(\lambda)$ or $\beta(\lambda)$.

Making use of Eq. (20), we conclude from Eq. (28) that

$I_{\lambda, 0}^{j} \eta_{\lambda}^{j} \simeq I_{\lambda, 0}^{j^{\prime}} \eta_{\lambda}^{j^{\prime}}$

The functions of wavelength $I_{\lambda, 0}^{j}$ and $\eta_{\lambda}^{j}$ strongly differ from one another (for instance $I_{\lambda, 0} \propto \lambda^{4 \pm 1}$ and $\eta_{\lambda} \simeq 1$ in the IR). A fortuitous compensation leading to Eq. (30) is quite unlikely. Thus Eq. (30) implies

$I_{\lambda, 0}^{j} \simeq I_{\lambda, 0}^{j^{\prime}}$

for any $j, j^{\prime}=1, m$, and

$\eta_{\lambda}^{j} \simeq \eta_{\lambda}^{j^{\prime}}$

Strictly speaking, the same values of parameters are needed in the corresponding model atmospheres, and the same effective temperature is assumed.

A rigorous treatment of this question seems beyond our grasp at present, at least until a sufficient number of resolved discs of carbon stars will be available. Consequently, we shall use Eq. (29) for our whole sample, including both uniform discs and totally-darkened discs of extended atmospheres, Eq. (14) being adopted. In the following sections, this conclusion is shown to be consistent with the predictions of model atmospheres.
Our understanding of the observations improved markedly with the analysis of Fig. 5 through Eq. (26), with coefficients as quoted in Table 6. These values will not be prescribed to $\mathrm{S} 2$ the whole sample of studied stars since artificial clusterings around a few values of $\Phi_{0}$ would result. We adopt $\alpha=1$ and Eqs. (28) and (29) instead, the latter being assumed to hold for every fixed value of the effective temperature. Finally Eq. (25) reduces to

$\log \Phi_{0}=\log \Phi-\log \langle k\rangle^{1 / 2}$

which allows the calibration of $\Phi_{0}\left(T_{\text {eff }}\right)$ (Sect. 11).

\section{The case of C5928 = TX Psc}

The low amplitude $\left(m_{\mathrm{pg}}=6,9-7.7\right)$ irregular $(\mathrm{Lb})$ variable TX Psc was frequently selected for a comparison of its observed SED to model atmospheres predictions. Lambert et al. (1986) found $T_{\text {eff }} \simeq 3030 \mathrm{~K}$ for TX Psc, with $\log g=0.0$ and $\mathrm{C} / \mathrm{O}=1.027$, comparing the observed and computed spectra in several wavelength ranges. Their model atmospheres however remained unpublished.

Our analysis has given CV2 and $E(B-V)=0.03$ (Paper III). The adopted angular diameter can be found in Table 2 together with the adopted direct effective temperature of $T_{\text {eff }} \simeq(3115 \pm 130) \mathrm{K}$ to be compared to the mean value of $T_{\text {eff }} \simeq(3130 \pm 70) \mathrm{K}$ as quoted in Table 3 for the CV2 group. Those values appear consistent.

Model atmospheres have been published for this carbon star with a $\mathrm{C} / \mathrm{O}$ ratio close to unity. The molecular bands being less strong than those exhibited by many carbon stars, confusions from line blends are a less severe problem in its spectrum. We concentrate here on models from Johnson et al. (1985), Jorgensen (1989), and Goebel et al. (1993). The reader is referred to these three papers for the detailed peculiarities of each of them. The authors usually compare their net fluxes $F_{\lambda}$ on a log scale to the published observations. We use instead

$\log s_{\lambda}=\log \left(F_{\lambda} / \pi B_{\lambda}\left(T_{\text {eff }}\right)\right)$

where $B_{\lambda}$ is the Planck function and $T_{\text {eff }}$ the assumed effective temperature. The spectral variations are thus more easily observable. The spectral resolution should be essentially the same in both SEDs. Predictions at high or moderate resolutions need to be integrated before comparison with spectrophotometric or even photometric SEDs. The necessary information is not fully available in every case. We must be ready to accept differences in "peaks and valleys" since the comparison relates to global spectral shapes over a large spectral range.

The SED we adopted in Sect. 4.1 for TX Psc was converted to $s_{\lambda}$ on a relative scale and adopting $T_{\text {eff }} \simeq$ $3115 \pm 130 \mathrm{~K}$ from Table 2 . It is compared in Fig. 6 to the model $T_{\text {eff }}=3000 \mathrm{~K}, \log g=0.0$ and $\mathrm{C} / \mathrm{O}=1.05$ of Johnson et al. (1985). The abundances they adopted are otherwise solar (their models with various hydrogen deficiencies are not considered here). The molecular opacities of $\mathrm{CN}, \mathrm{CH}, \mathrm{C}_{2}$ and $\mathrm{CO}$ are included in the model 


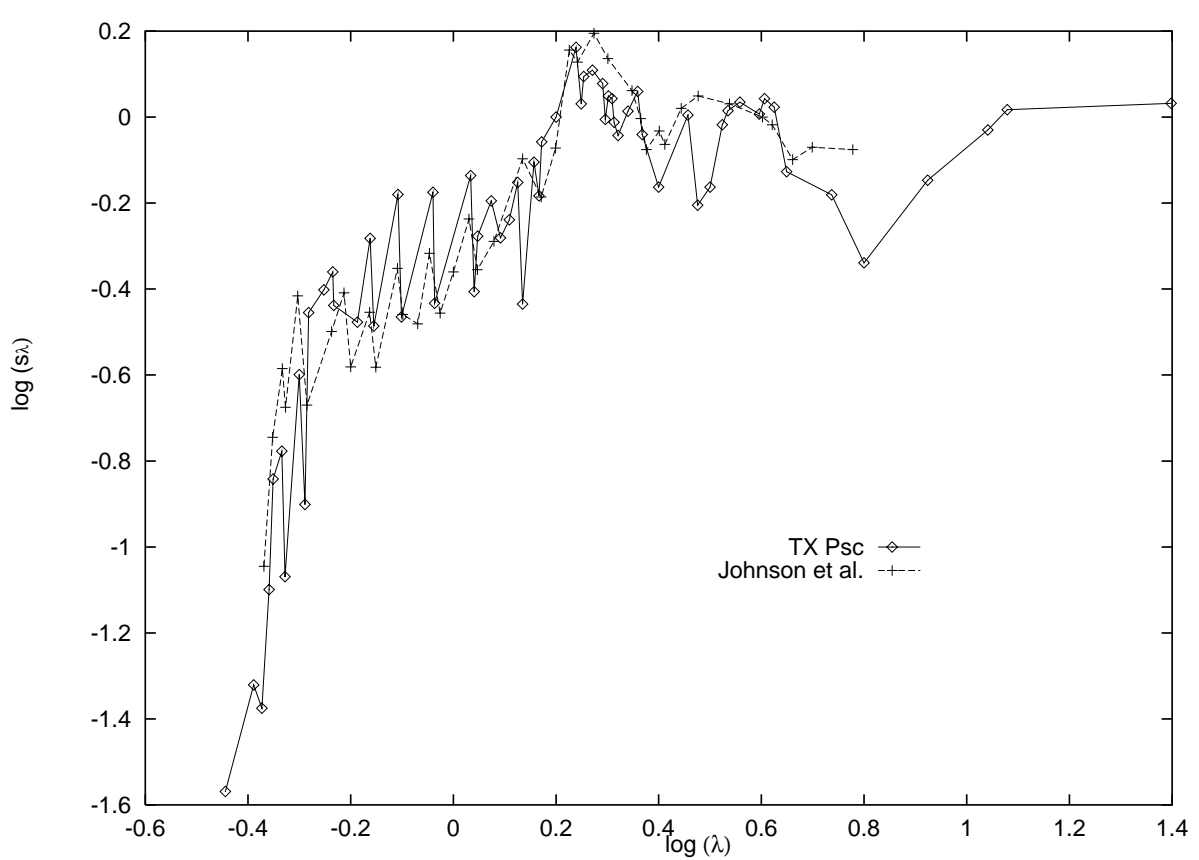

Fig. 6. The comparison of the SED of TX Psc adopting $T_{\text {eff }}=3115 \mathrm{~K}$ with the predictions from the model $T_{\text {eff }}=3000 \mathrm{~K}$, $\log g=0.0$ and $C / 0=1.05$ of Johnson et al. (1985). See text for details

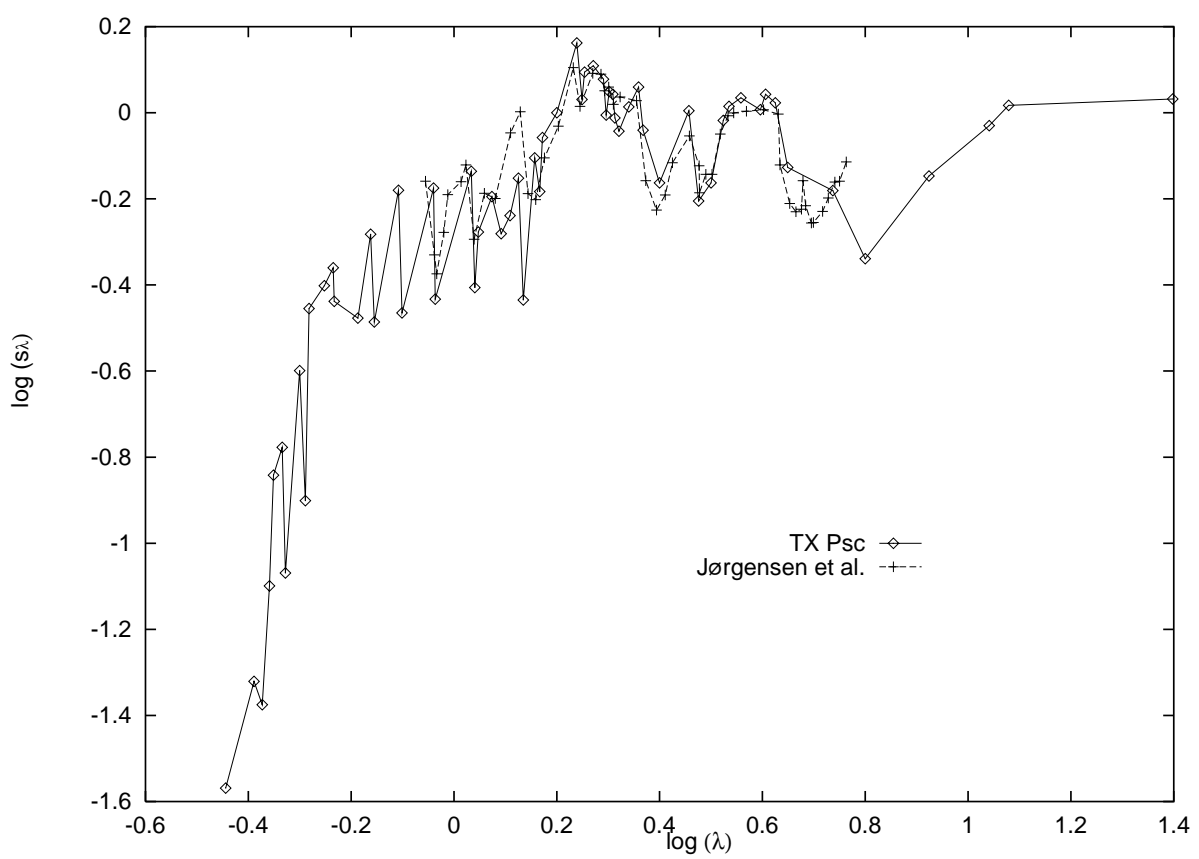

Fig. 7. The comparison of the SED of TX Psc adopting $T_{\text {eff }}=3115 \mathrm{~K}$ with the predictions of the model $T_{\text {eff }}=3100 \mathrm{~K}$, $\log g=-0.5$ and $C / 0=1.023$ of Jorgensen (1989). See text for details

while those of $\mathrm{HCN}$ and $\mathrm{C}_{2} \mathrm{H}_{2}$ are not. This is the reason why their SED is free from the strong dip noticed around $\lambda \simeq 3.1 \mu \mathrm{m}$. The latter bands are however responsible for strong blanketing which possibly affects the whole $2.8-3.5 \mu \mathrm{m}$ region. The "ups and downs" below $1.0 \mu \mathrm{m}$ illustrate the influence of the red system of CN. They are much fainter in the model than in the observed SED.

We can see that the global shape of the two SEDs in Fig. 6 are much the same from UV to IR. The drop observed toward short wavelengths can be ascribed to a strong increase in opacities. The slight shift noticed between both curves at $\log \lambda<-0.2$ is negligible when $T_{\text {eff }}$ is varied from 2985 to $3245 \mathrm{~K}$. A discrepancy is apparent in the former case for $\log \lambda<0$, while the SED obtained in the latter case is inconsistent with the model. In addition, the values assumed for $\log g$ and $\mathrm{C} / \mathrm{O}$, and solar abundances may be at variance with the true values. A temperature such as $3050 \mathrm{~K} \leq T_{\text {eff }} \leq 3115 \mathrm{~K}$ is most likely from this global comparison. We note that the 3115-value was deduced from the observed angular 
diameter as adopted in Sect. 5 on the assumption of Eq. (14), i.e. of $\Phi_{\mathrm{UD}}$ and $\eta_{\lambda} \simeq 1$ in Eq. (20). We also modified Fig. 6, adopting $\Phi_{\mathrm{FDD}} \simeq 11.4$ mas which would correspond to $T_{\text {eff }} \simeq 2815 \mathrm{~K}$ and $\eta_{\lambda} \simeq 0.82$. The strong decrease toward short wavelengths then almost vanishes. This low effective temperature that one would obtain on the FDD assumption is close to the mean color temperature $T_{\mathrm{C}} \simeq 2750 \pm 50 \mathrm{~K}$ in the visible and infrared. For $\eta_{\lambda} \simeq 0.91$ we get $\Phi_{\mathrm{LD}} \simeq 10.24$ mas and $T_{\text {eff }} \simeq 2970 \mathrm{~K}$ close to $2985 \mathrm{~K}$ for which moderate discrepancies were noted. Finally, $3050 \mathrm{~K} \leq T_{\text {eff }} \leq 3120 \mathrm{~K}$ is favored from this comparison, and a slight effect of darkening and/or extension can be present.

The same SED as normalized above in Fig. 6 is compared in Fig. 7 with the predictions of the model $T_{\text {eff }}=$ $3100 \mathrm{~K}, \log g=-0.5$ and $\mathrm{C} / \mathrm{O}=1.023$ of Jorgensen (1989). Unfortunately, the SED published for this model is limited to the $\lambda \geq 0.88 \mu \mathrm{m}$ range. This is certainly the best fit obtained in the $0.88 \mu \mathrm{m} \leq \lambda \leq 5.2 \mu \mathrm{m}$ spectral range, except for a noticeable discrepancy in the $1.30-1.34 \mu \mathrm{m}$ interval (possibly due to differences in $\mathrm{C}_{2}$ Ballik-Ramsay band intensities). The bands of $\mathrm{CN}, \mathrm{HCN}$ etc. around $2.5 \mu \mathrm{m}$ and $3.1 \mu \mathrm{m}$ are fairly well reproduced. The two bandheads of the red CN-system close to $1.0 \mu \mathrm{m}$ are in much better agreement than those of Johnson et al. (1985). We also compared this model with the observed SED adopting $T_{\text {eff }}=3245 \mathrm{~K}$ (no agreement for $\lambda \leq 1.4 \mu \mathrm{m}$ ) and $T_{\text {eff }}=2985 \mathrm{~K}$ (almost satisfactory). A temperature slightly less than $T_{\text {eff }}=3115 \mathrm{~K}$ might yet be acceptable.

The comparison with the model $T_{\text {eff }}=3030 \mathrm{~K}$, $\log g=0.0$ of Goebel et al. (1993) for $\lambda \geq 1.0 \mu \mathrm{m}$, is not shown here. It provides a poorer agreement. The spectral shape is almost satisfactory but the poly-atomic bands $\mathrm{HCN}+\mathrm{C}_{2} \mathrm{H}_{2}$ are too strong at 2.5, 3.1, and $3.8 \mu \mathrm{m}$.

In conclusion, we keep here the value $T_{\text {eff }}=3115 \mathrm{~K}$ for TX Psc despite some indication from the above comparisons to reduce it slightly $(\simeq 30 \mathrm{~K})$. It is close to the $3130 \pm 70 \mathrm{~K}$ obtained for the group CV2 and quoted in Table 3. The precision to be expected from such comparisons is not high enough to pinpoint the temperatures. The various uncertainties on opacities, atmospheric structure, and values of the other parameters, need be taken into account. The use of $\Phi_{\mathrm{UD}}$ is confirmed to be an acceptable approximation for this kind of star.

\section{The mean SEDs of the photometric groups and model atmospheres predictions}

According to the approach described in Sect. 3, we compared the overall spectral shapes in the mean SEDs of the photometric groups to the SEDs available from model atmospheres. The effective temperatures derived for CV1, $\mathrm{CV} 2$ and CV3 were of $T_{\text {eff }}=3240 \mathrm{~K}, 3050 \mathrm{~K}$ and $2950 \mathrm{~K}$ respectively in the comparisons to the models from references cited in Sect. 9. The estimated accuracy should be slightly better than $\pm 100 \mathrm{~K}$, possibly $\pm 80 \mathrm{~K}$. These results are consistent with the mean direct effective temperatures
Table 7. Mean effective temperatures (in K) from observed angular diameters (Sect. $5, T_{\text {eff } 1}$ ), from the comparison of mean SEDs to model atmosphere predictions (Sects. 9 and $10, T_{\text {eff2 }}$ ), and from a batch of five calibrated color indices (Sect. $7, T_{\text {eff } 3}$ ). Provisionally adopted values $\left(T_{\mathrm{eff}}\right)$ are also quoted

\begin{tabular}{ccccc}
\hline Group & $T_{\text {eff1 }}$ & $T_{\text {eff2 }}$ & $T_{\text {eff3 }}$ & $T_{\text {effp }}$ \\
\hline HC0 & & & 5730 & 5730 \\
HC1 & & $4710 \pm 200$ & 4830 & 4770 \\
HC2 & & $4250 \pm 150$ & 4420 & 4335 \\
HC3 & & $4020 \pm 100$ & 4175 & 4100 \\
HC4 & & $3800 \pm 100$ & 3890 & 3845 \\
HC5 & & $3500 \pm 100$ & 3440 & 3470 \\
CV1 & $3415 \pm 640$ & $3240 \pm 100$ & 3170 & 3205 \\
CV2 & $3120 \pm 70$ & $3050 \pm 100$ & 3000 & 3060 \\
CV3 & & $2950 \pm 100$ & 2860 & 2910 \\
CV4 & $2710 \pm 100$ & & 2770 & 2740 \\
CV5 & $2750 \pm 100$ & & 2630 & 2690 \\
CV6 & $2385 \pm 110$ & & 2460 & 2420 \\
CV7max & $2000 \pm 100$ & & 2080 & 2040 \\
CV7min & $1820 \pm 100$ & & & 1820 \\
\hline
\end{tabular}

as quoted in Table 3. We used older models from Querci et al. (1974) and Querci \& Querci (1976) for the hotter $\mathrm{HC}$-stars. The value $\mathrm{C} / \mathrm{O} \simeq 3.5$ they adopted seems too high when compared to the results of Lambert et al. (1986) for the $\mathrm{CV}$ stars $(\mathrm{C} / \mathrm{O} \leq 2.0)$. Here, we however deal with HC-stars for which Vanture (1992) obtained $1 \leq \mathrm{C} / \mathrm{O} \leq 8$ from spectral analysis.

The same $\log \lambda-\log s_{\lambda}$ diagram is used in Fig. 8 for the mean (observed) SED of the HC1-group with $T_{\text {eff }}=4710 \mathrm{~K}$ assumed, which yields the best agreement with the DE12 model of Querci et al. (1974) with $T_{\text {eff }}=4500 \mathrm{~K}$ and $\log g=-1.0$. From various trials, we thus adopt $T_{\text {eff }} \simeq 4710 \pm 200 \mathrm{~K}$ as the mean effective temperature for the $\mathrm{HC} 1$ group. Contrary to the case of TX Psc (Sect. 9) which is a CV2 star, the opacity on a relative scale substantially increases only in the ultraviolet. Intermediate behaviors are observed on the interval $\mathrm{HC} 1$ to $\mathrm{CV} 2$, the slopes and curvatures variations being essential for effective temperature evaluations. Consistent with Sect. 3, we did not attempt detailed fits of the individual molecular bandheads. The comparison is shown in Fig. 9 of the mean SED of HC5 $\left(T_{\text {eff }}=3500 \mathrm{~K}\right)$ to the predictions for the DE12 model at $T_{\text {eff }}=3400 \mathrm{~K}$ and $\log g=-1.0$ of Querci et al. (1974). The global shape is satisfactorily reproduced but discrepancies in the details are noticed. We have also attempted comparisons for the other $\mathrm{CV}$-groups (CV3 to CV7). The discrepancies increase when models of $T_{\text {eff }}=2600 \mathrm{~K}$ or less from various authors, are used. Clearly the effect of the detailed opacities from polyatomic species and eventually grains, are not fully taken into account in the coolest models. We conclude there is a rough agreement with the mean values as quoted in Table 3, but further investigations with more realistic models are necessary to fully assess the cool end of the 


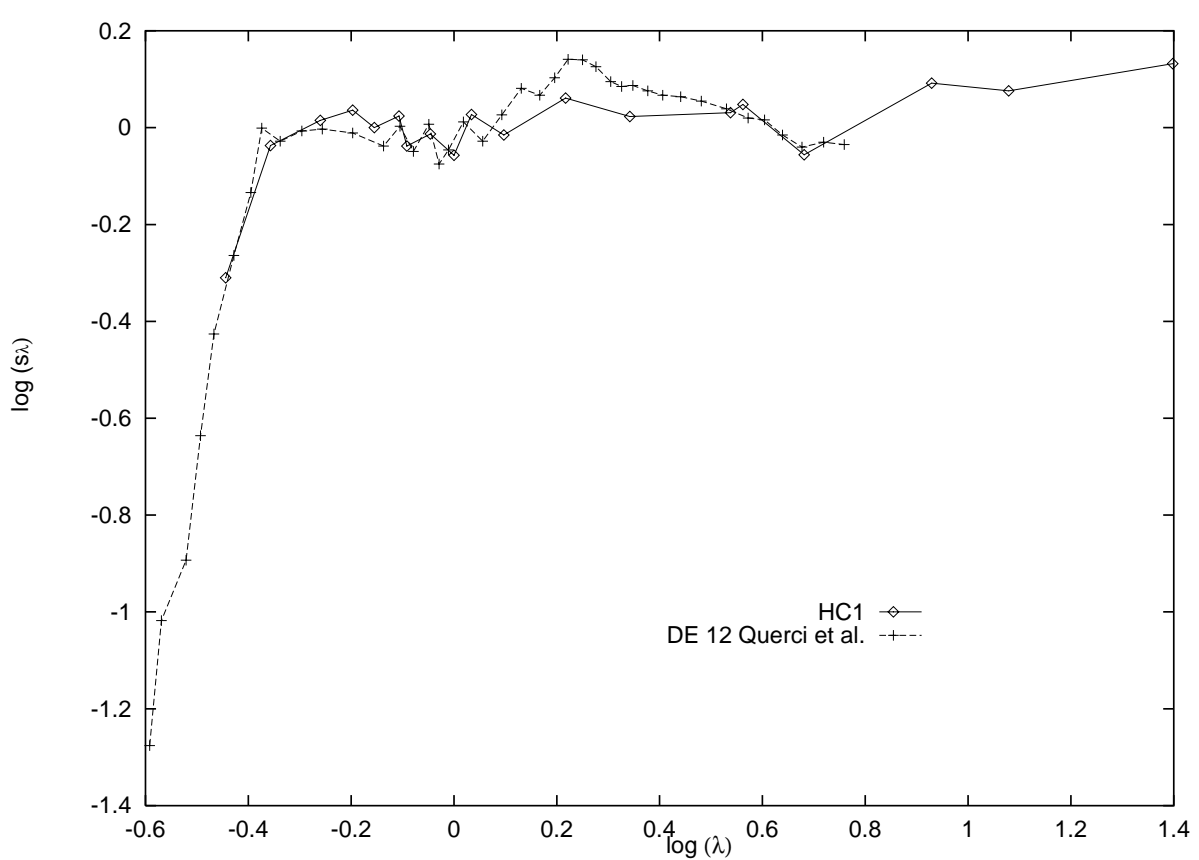

Fig. 8. The comparison of the mean HC1-SED adopting $T_{\text {eff }}=4710 \mathrm{~K}$, with the predictions of the DE12 model $T_{\text {eff }}=4500 \mathrm{~K}$, $\log g=-1.0$ of Querci et al. (1974)

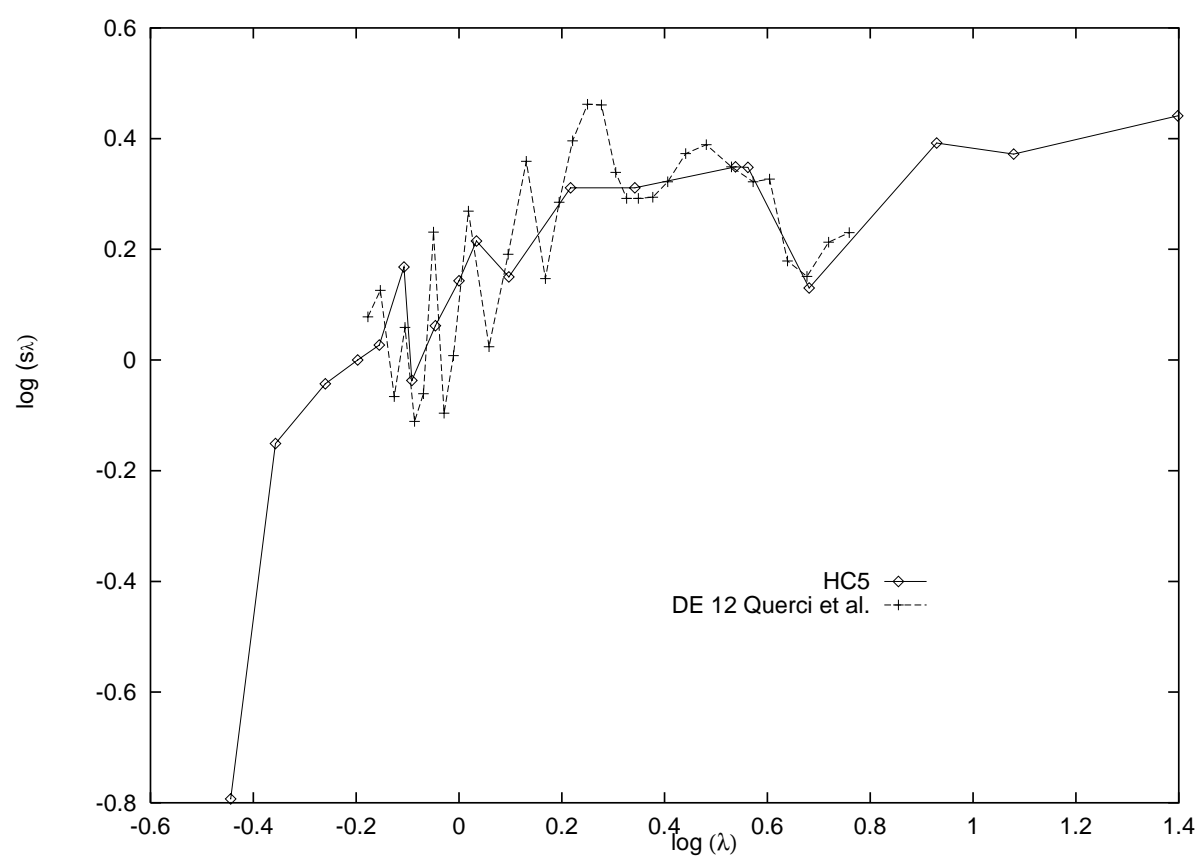

Fig. 9. The comparison of the mean HC5-SED adopting $T_{\text {eff }}=3500 \mathrm{~K}$, with the predictions of the DE12 model $T_{\text {eff }}=3400 \mathrm{~K}$, $\log g=-1.0$ of Querci et al. (1974)

sequence. We have collected in Table 7 the best estimates of effective temperatures from the three methods used

- direct values from occultation and/or interferometric diameters (Table 3);

- SED comparisons between observations and models;

- batch of five calibrated color indices (Eq. (19) and Table 5), with extrapolation to higher temperatures.
A very satisfactory agreement is found between these three methods and provisional mean effective temperatures are quoted in the last column of Table 7.

\section{Reference angular diameters $\Phi_{0}$ and coefficients $\mathrm{C}_{T_{\text {eff }}}$}

The reference angular diameter $\Phi_{0}$ was introduced through Eq. (23) in Sect. 8. It is the angular diameter an "identical" source with $[1.08]_{0}=0$, or equivalently 
$\langle k\rangle^{1 / 2}=1$, would have. By identical we mean displaying the same intensity distribution over the disc (see Sect. 8). Assuming this is the case for the stars belonging to the same photometric group, we derived the mean coefficients of Table $6, \Phi_{0}$ being deduced from $\beta$ through Eq. (29). Following the results obtained in Sect. 8 and the arguments developed there, we adopt $\alpha=1$ and

$\log \Phi_{0}=\log \Phi-\log \langle k\rangle^{1 / 2}$

for each of the fifty-four SEDs associated to measured angular diameters (Table 2$)$. The diagram $\Phi_{0}\left(T_{\text {eff }}\right)$ adopting the direct values of Table 2, is shown as Fig. 10 where the six points from Table 6, i.e. Eq. (29), were added. The obtained function is monotonically decreasing with increas$\operatorname{ing} T_{\text {eff }}$, except for a small bump near $\log T_{\text {eff }} \simeq 3.385$ i.e. at about $2400 \mathrm{~K}$ which is close to the elbow of Sect. 7 (namely $2300 \mathrm{~K}$ ). Both phenomena might have the same cause. The main trend (monotone decrease) was expected since the integrated intensity increases with the effective temperature, and then a smaller $\Phi_{0}$ is required to produce the same $[1.08]_{0}=0$. We have no direct value at hand for the hot carbon stars of the HC-groups. The integrated flux $F_{0}$ the star would radiate if $[1.08]_{0}=0$, may however be used in an indirect way together with the provisional mean effective temperatures of Table 7 . We define now

$C_{T_{\text {eff }}}=\left(\Phi_{0} / 2\right)\left(T_{\text {eff }} / T_{\text {eff } \odot}\right)^{2}$.

This is the reference angular radius the star would show if its effective temperature amounted to that of the sun. It can be expressed in mas and then

$C_{T_{\text {eff }}}=2.06310^{8}\left(F_{0} / \sigma T_{\text {eff } \odot}^{4}\right)^{1 / 2} \simeq\left(6.7710^{12} F_{0}\right)^{1 / 2}$

with $T_{\text {eff } \odot} \simeq 5770 \mathrm{~K}$. Thus, with $F_{0}$ in Watt $\mathrm{cm}^{-2}$ and $T_{\text {eff }}$ in $\mathrm{K}$, the reference diameter $\Phi_{0}$ is estimated from

$\Phi_{0}=2\left(6.7710^{12} F_{0}\right)^{1 / 2} /\left(T_{\text {eff }} / T_{\text {eff } \odot}\right)^{2}$.

We can transform Eq. (36) into the intermediate formula

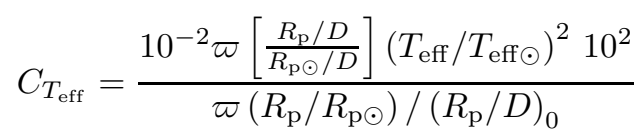

where the estimated true parallaxe $\varpi$ is also expressed in mas. Then, from Eq. (39), we derive an equivalent expression for $C_{T_{\text {eff }}}$ in terms of observable quantities. Introducing the stellar luminosity in solar units, the distance modulus relation can be written as

$10^{-0.2\left(m_{\mathrm{bol}}-M_{\mathrm{bol} \odot}\right)}=10^{\log \varpi-2+0.5 \log \left(L_{*} / L_{\odot}\right)}$.

We obtain after some rearrangement

$10^{-0.2\left(m_{\mathrm{bol}}-M_{\mathrm{bol} \odot}\right)}=10^{-2} \varpi \frac{R_{\mathrm{p}} / D}{R_{\mathrm{p} \odot} / D}\left(T_{\text {eff }} / T_{\text {eff } \odot}\right)^{2}$

which is the numerator of (39) divided by $10^{2}$, while its denominator can be written as

$$
\begin{array}{r}
\langle k\rangle^{1 / 2} /\left(R_{\mathrm{p} \odot}\right)_{\mathrm{AU}} \simeq\langle k\rangle^{1 / 2} / 2.06310^{5}\left(R_{\mathrm{p} \odot}\right)_{\mathrm{pc}} \\
\simeq 214.94\langle k\rangle^{1 / 2}=(\varpi)_{\operatorname{mas}} \frac{R_{\mathrm{p}} / R_{\mathrm{p} \odot}}{\left(R_{\mathrm{p}} / D\right)_{0}}
\end{array}
$$

Replacing Eqs. (41) and (42) into Eq. (39), we finally obtain

$C_{T_{\text {eff }}}=\frac{10^{-0.2\left(m_{\mathrm{bol}}-M_{\mathrm{bol} \odot}\right)+2}}{214.94\langle k\rangle^{1 / 2}}$

which requires the knowledge of the apparent bolometric magnitude of the star $m_{\text {bol }}$ and that of the relative angular diameter $\langle k\rangle^{1 / 2}$, both quantities being deduced from our photometric analysis. The $F_{0}$ values were calculated for every photometric group, i.e. from its averaged SED with $[1.08]_{0}=0$. The fluxes obtained through integration of the SEDs with 19 points were corrected (see Sect. 4.1) with the coefficient $r_{1}$ (category I, i.e. 0.97 for HC3 to CV6 and $\mathrm{SCV}$, or II, i.e. 0.88 for $\mathrm{CV} 7$ ) or unity (no correction for $\mathrm{HC} 0$ to $\mathrm{HC} 2$ ). The J-stars also require $r_{1}=0.88$ but they are rare among the $\mathrm{HC}$ and $\mathrm{CV}$-groups. In practice, we derived the bolometric reference magnitudes $m_{\mathrm{bol}, 0}$ from

$m_{\mathrm{bol}}-m_{\mathrm{bol}, 0}=[1.08]_{0}$

and the reference flux $F_{0}$ in Watt $\mathrm{cm}^{-2}$ from

$m_{\mathrm{bol}, 0}=-2.5 \log \left(F_{0} / 2.49710^{-12}\right)$.

The $C_{T_{\text {eff }}}$ values were deduced from Eq. (37). Adopting

$T_{\text {eff }} \simeq T_{\text {effp }}$

estimates of $\Phi_{0}\left(T_{\text {effp }}\right)$ were derived from Eq. (38). The results are quoted in Table 8 and plotted in Fig. 10 as squared symbols. The $\Phi_{0}\left(T_{\text {eff }}\right)$ relation is thus extended toward higher temperatures. It would be helpful to observe a few angular diameters of hot carbon (HC) stars to corroborate this provisional calibration.

It can be seen in Fig. 10 that there is a good consistency between the reference angular diameters following our three approaches, i.e. as calculated from

- the direct mean values obtained from Eq. (29) applied to $\left\langle\Phi_{0}\right\rangle$ the mean values of Table 6 ;

- the individual values for 54 measured angular diameters derived from Eq. (35);

- the mean values for the groups $\left\langle\Phi_{0}\left(T_{\text {effp }}\right)\right\rangle$ in Table 8 , as deduced from Eq. (38), adopting Eq. (46) with values taken from Table 7 .

Finally, we deduce six linear fits for interpolation of $\log \Phi_{0}$ against $\log T_{\text {eff. }}$.

$\log \Phi_{0}=\gamma \log T_{\text {eff }}+\delta$

from the method of least squares. They are given in Table 9, together with those of

$\log C_{T_{\text {eff }}}=\gamma^{\prime} \log T_{\text {eff }}+\delta^{\prime}$

after Eq. (36) was applied. They are shown in Fig. 11. The major drawback of this latter function is that it is not monotone: a minimum is observed at roughly $3500 \mathrm{~K}$ i.e. inside the HC5-group. It also appears as more dispersed than the $\Phi_{0}\left(T_{\text {eff }}\right)$ relation which should be preferred. 
Table 8. Mean effective temperatures, reference fluxes and reference apparent bolometric magnitudes of the 14 photometric groups. The additional quantity $C_{T_{\text {eff }}}$ and the reference mean angular diameter $\Phi_{0}$ are also quoted, both of them being expressed in mas. The number of stars per group is denoted by $n$ (for a total of 388 stars) and the mean color temperature obtained as described in Sect. 15, are compared to the mean effective temperatures derived by the method of Sect. 12

\begin{tabular}{ccccccccc}
\hline Group & $\left(T_{\text {effp }}\right)$ & $\left\langle F_{0}\right\rangle \mathrm{Wcm}^{-2}$ & $\left\langle m_{\text {bol,0 }}\right\rangle$ & $\left\langle C_{T_{\text {eff }}}\right\rangle$ & $\left\langle\Phi_{0}\right\rangle$ & $n$ & $\left\langle T_{\text {eff }}\right\rangle$ & $T_{\mathrm{c}}$ \\
\hline HC0 & 5730 & $1.1210^{-12}$ & 0.869 & 2.755 & 6.00 & 4 & 5620 & $5800:$ \\
HC1 & 4770 & $8.3010^{-13}$ & 1.196 & 2.371 & 7.07 & 27 & 4890 & 4530 \\
HC2 & 4335 & $7.8010^{-13}$ & 1.263 & 2.298 & 8.47 & 27 & 4290 & 4050 \\
HC3 & 4100 & $7.8610^{-13}$ & 1.221 & 2.307 & 9.27 & 16 & 4005 & 3810 \\
HC4 & 3845 & $7.2110^{-13}$ & 1.314 & 2.209 & 10.03 & 14 & 3965 & 3520 \\
HC5 & 3470 & $6.8910^{-13}$ & 1.398 & 2.159 & 11.94 & 20 & 3480 & 3030 \\
CV1 & 3205 & $7.0110^{-13}$ & 1.378 & 2.179 & 14.12 & 36 & 3285 & 2850 \\
CV2 & 3060 & $7.7610^{-13}$ & 1.268 & 2.293 & 16.31 & 47 & 3035 & 2720 \\
CV3 & 2910 & $7.9810^{-13}$ & 1.239 & 2.324 & 18.46 & 44 & 2915 & 2600 \\
CV4 & 2740 & $8.3210^{-13}$ & 1.193 & 2.373 & 21.05 & 32 & 2775 & 2480 \\
CV5 & 2690 & $8.8810^{-13}$ & 1.123 & 2.452 & 22.56 & 45 & 2645 & 2410 \\
CV6 & 2420 & $1.0010^{-12}$ & 0.991 & 2.605 & 29.62 & 47 & 2445 & 2280 \\
CV7 & 2040 & $1.8010^{-12}$ & 0.354 & 3.492 & 58.13 & 19 & $1955^{\mathrm{a}}$ & 1850 \\
SCV & 2820 & $8.1210^{-13}$ & 1.220 & 2.344 & $19.63:$ & 10 & 2775 & \\
\hline
\end{tabular}

${ }^{\text {a }}$ Variables mostly close to maximum or at intermediate phase (2000 K) with only a few stars close to minimum (1750 K).

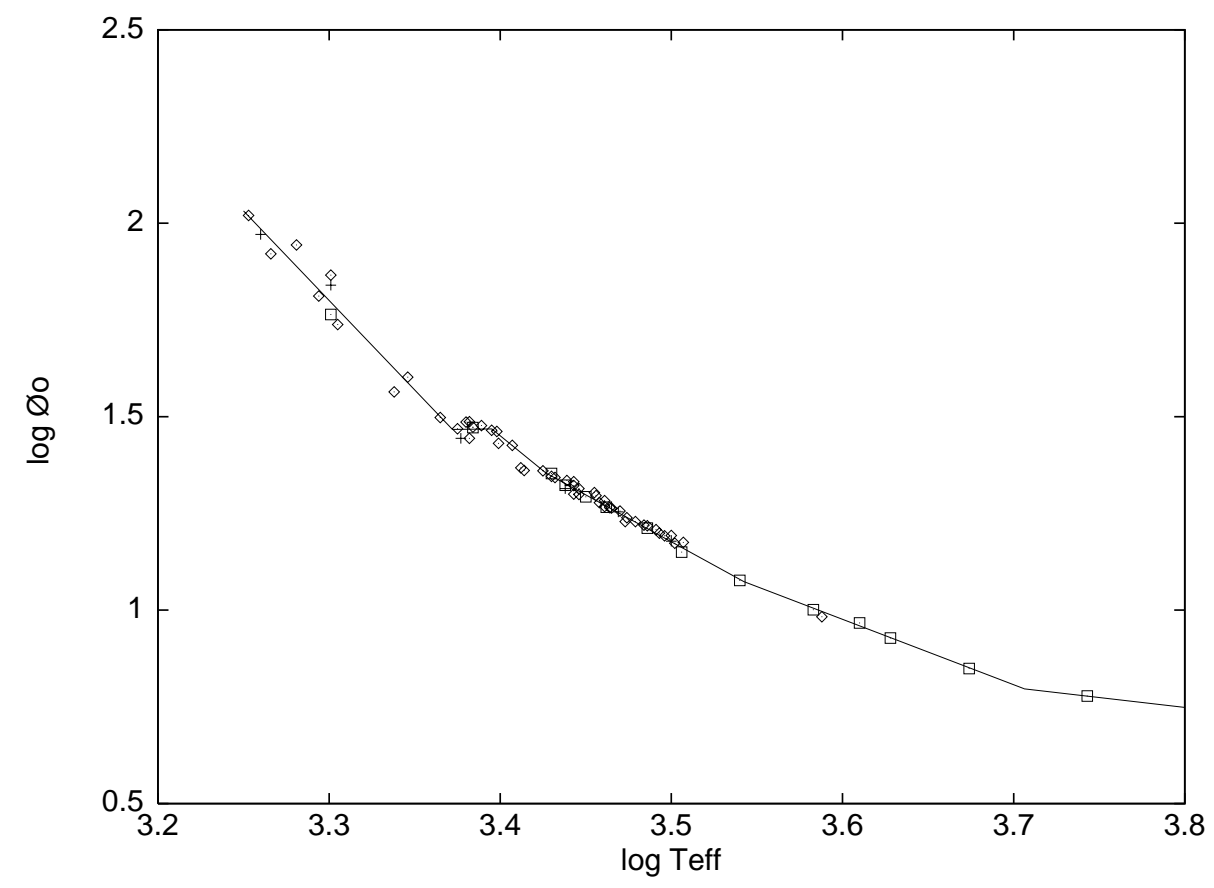

Fig. 10. The reference angular diameters as plotted against the effective temperatures, both on a log scale. Diamonds correspond to direct individual values for the 54 observations collected, the crosses to the 6 direct mean values of Table 6 , and the squares to the estimates from Eq. (38) with $T_{\text {effp }}$ from Table 7 . Six contiguous linear fits are shown

\section{A new homogeneous effective temperature scale for carbon stars}

The next step is to evaluate the effective temperature of the carbon stars whose angular diameter was not measured. We use definition (36) with $C_{T_{\text {eff }}}$ calculated from
Eq. (37). The function in (36) is then transcendental in $T_{\text {eff }}$ with $\Phi_{0}\left(T_{\text {eff }}\right)$ given as a numerical function (47) with coefficients as quoted in Table 9 . A second method is the use of the five color indices calibrations in Table 5. The estimates may vary with the selected index, especially at high temperature. Their mean is however close to the value 


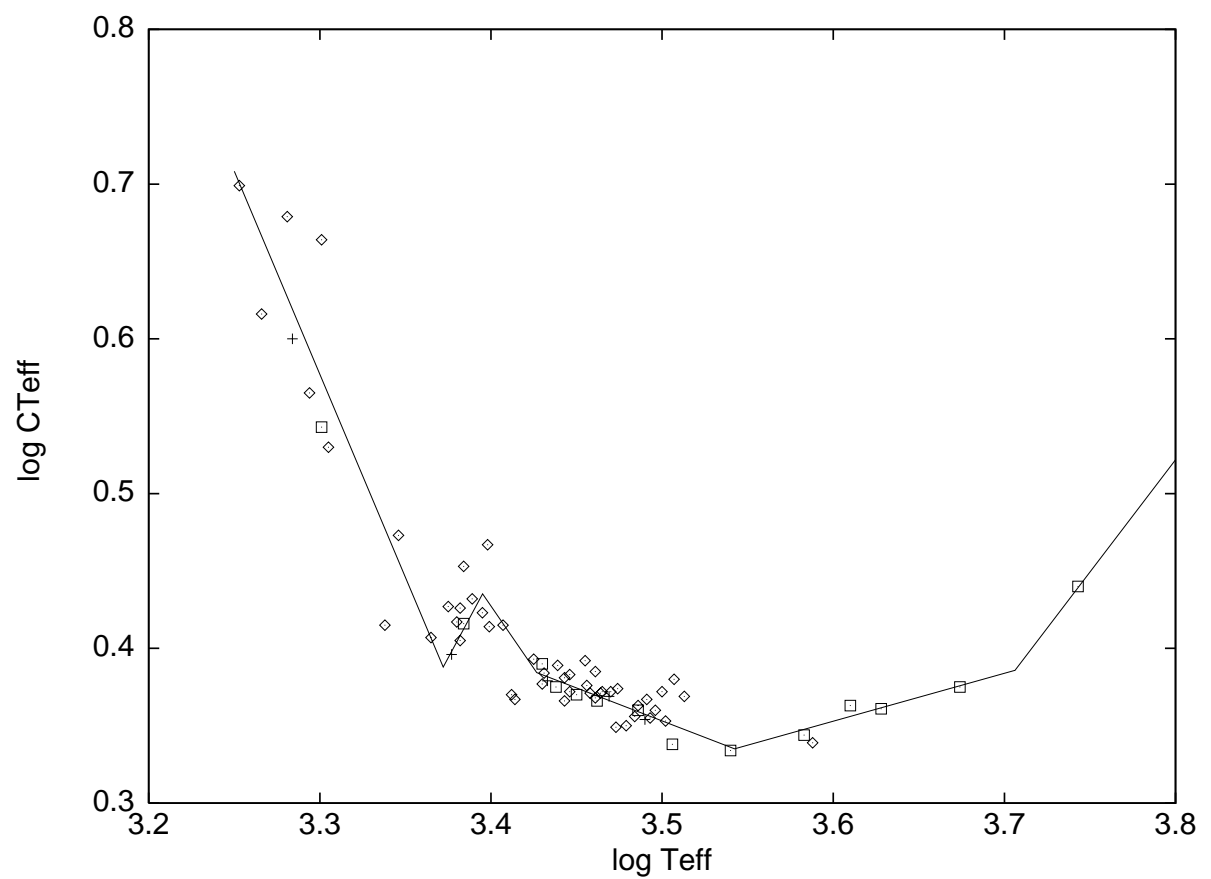

Fig. 11. The same figure as Fig. 10 for the $C_{T_{\text {eff }}}$-coefficient as derived from Eq. (36), the symbols being the same

Table 9. The interpolation relations as fitted through data of Fig. 10 of $\log \Phi_{0}$ and $\log C_{T_{\text {eff }}}$ as a function of $\log T_{\text {eff }}$. The least-squares method was used to derive six linear fits according to Eq. (47). The coefficients in Eq. (48) are from Eq. (36)

\begin{tabular}{ccccc}
\hline $\log T_{\text {eff }}$ range & $\gamma$ & $\delta$ & $\gamma^{\prime}$ & $\delta^{\prime}$ \\
\hline$\leq 3.37$ & -4.625 & 17.062 & -2.625 & 9.238 \\
$3.37-3.39$ & 0.052 & 1.292 & 2.052 & -6.532 \\
$3.39-3.43$ & -3.609 & 13.722 & -1.609 & 5.898 \\
$3.43-3.54$ & -2.426 & 9.666 & -0.426 & 1.842 \\
$3.54-3.70$ & -1.690 & 7.062 & 0.310 & -0.762 \\
$\geq 3.70$ & -0.515 & 2.705 & 1.485 & -5.118 \\
\hline
\end{tabular}

derived through the former method. The final calibrations of indices as quoted in Table 11, should be preferred, especially when using the indices separately, and/or when dealing with (hot) HC-stars. Our new homogeneous scale for carbon stars makes use of both methods, viz.

- the former, using Eq. (36), ultimately relies on integrated fluxes $F_{0}$ for $[1.08]_{0}=0$ and leads to $\left(T_{\text {eff }}\right)_{F_{0}}$;

- the latter, based on SEDs as described by a batch of five color indices, which yields $\left(T_{\text {eff }}\right)_{\mathrm{CI}}$.

The consistency of these two values is then checked and their arithmetic mean adopted unless some substantial difference occurs, either explained or not. These are not independent determinations since they both rely on measured angular diameters for their calibrations. The new scale is however confirmed independently by model atmosphere predictions, at least for $T_{\text {eff }} \geq 2800 \mathrm{~K}$.

It is expected that individual values are accurate to better than $\pm 70 \mathrm{~K}$ or $\pm 140 \mathrm{~K}$ in the worst cases (internal errors only). The large amplitude variables (Miras, SR)
Table 11. Unreddened mean color indices and mean effective temperatures of the fourteen photometric groups. The indices are respectively $I_{1}=(V-[1.08])_{0}, I_{2}=(V-K)_{0}$, $I_{3}=([1.08]-K)_{0}, I_{4}=(J-K)_{0}$, and $I_{5}=(H-K)_{0}$

\begin{tabular}{cccccccc}
\hline Gr. & $\left\langle T_{\text {eff }}\right\rangle$ & $\log \left\langle T_{\text {eff }}\right\rangle$ & $I_{1}$ & $I_{2}$ & $I_{3}$ & $I_{4}$ & $I_{5}$ \\
\hline HC0 & 5620 & 3.7497 & 0.74 & 1.05 & 0.31 & 0.23 & 0.11 \\
HC1 & 4890 & 3.6893 & 1.51 & 2.23 & 0.72 & 0.58 & 0.12 \\
HC2 & 4290 & 3.6325 & 1.83 & 2.81 & 0.98 & 0.77 & 0.18 \\
HC3 & 4005 & 3.6026 & 2.02 & 3.19 & 1.17 & 0.87 & 0.23 \\
HC4 & 3965 & 3.5982 & 2.44 & 3.67 & 1.23 & 0.97 & 0.28 \\
HC5 & 3480 & 3.5416 & 3.11 & 4.53 & 1.42 & 1.21 & 0.35 \\
CV1 & 3285 & 3.5165 & 3.45 & 5.04 & 1.59 & 1.42 & 0.43 \\
CV2 & 3035 & 3.4822 & 3.73 & 5.47 & 1.74 & 1.47 & 0.47 \\
CV3 & 2915 & 3.4646 & 3.86 & 5.71 & 1.85 & 1.63 & 0.53 \\
CV4 & 2775 & 3.4433 & 4.06 & 5.97 & 1.91 & 1.67 & 0.55 \\
CV5 & 2645 & 3.4224 & 4.27 & 6.32 & 2.05 & 1.74 & 0.61 \\
CV6 & 2445 & 3.3883 & 4.42 & 6.67 & 2.25 & 1.97 & 0.68 \\
CV7 & 1955 & 3.2911 & 5.01 & 7.92 & 2.91 & 2.44 & 0.94 \\
SCV & 2775 & 3.4433 & 4.45 & 6.32 & 1.87 & 1.44 & 0.37 \\
\hline
\end{tabular}

exhibit frequently variable effective temperatures depending on the adopted SED and corresponding phase. The correlation between effective temperatures and photometric groups is here again confirmed with the highest temperatures obtained close to maximum light at the earliest $\mathrm{HC}$ or CV-group displayed by the variable. The less accurate temperatures are presumably located

- at the "hot" (HC0) and "cool" (CV7) ends;

- in the intermediate HC5-zone near $3500 \mathrm{~K}$ which corresponds to the flat minimum of $C_{T_{\text {eff }}}$ in Fig. 11.

A sample of more than 400 carbon stars has been analyzed for effective temperatures on the new homogeneous 
scale. Together with Ba II and related ${ }^{1}$ stars, and carbon stars classified with oxygen-types SEDs, whose temperatures were deduced from published calibrations (Knapik 1999), this is an enlarged sample of more than 500 stars which has been studied for effective temperatures. The detailed results and photometric solutions are given in Table 10, available at CDS (Strasbourg). A condensed version is given in the Appendix. The mean values on the new scale are quoted for every photometric group in Table 8, as $\left\langle T_{\text {eff }}\right\rangle$ (Col. 8). They appear close to the provisional $T_{\text {effp }}$ values of Col. 2. They are consistent with the temperatures $T_{\text {eff2 }}$ (Table 7 ) derived through comparisons of observed SEDs to model atmospheres predictions.

The carbon stars with optically thick circumstellar shells are a special case since the $K$-magnitudes may be strongly affected by circumstellar emission and only $I_{1}=(V-[1.08])_{0}$ remained usable. We add at last $(V-[0.78])_{0}$ and $(V-J)_{0}$, to estimate their $\left(T_{\text {eff }}\right)_{\mathrm{CI}}$ values. We note that the $m_{\text {bol }}$ values (Tables 10 and A.1), are determined from the whole SED after dereddening for the selective extinction, following the usual standard interstellar extinction law (Papers I to III).

Thus a circumstellar contribution may well be included. Eventual neutral extinctions as discussed in Papers II and III, remain undetected except for possible emission counterparts in the IR. At long wavelengths where the selective extinction decreases the contribution of grain emission is included in the observed net flux. The estimate $\left(T_{\text {eff }}\right)_{F_{0}}$ is also affected since it is obtained from $F_{0}$ and Eq. (37). In the overwhelming majority of our stars, we obtained

$\left(T_{\text {eff }}\right)_{F_{0}} \simeq\left(T_{\text {eff }}\right)_{\mathrm{CI}}$.

Whenever this was not true, the corresponding star deserved a separate study. The ten cases we considered most prominent in our data are discussed in Sect. 13.

We have checked for a possible systematic shift between the results from the two methods, by calculating

$\left\langle\frac{2\left[\left(T_{\text {eff }}\right)_{\mathrm{CI}}-\left(T_{\text {eff }}\right)_{F_{0}}\right]}{\left(T_{\text {eff }}\right)_{\mathrm{CI}}+\left(T_{\text {eff }}\right)_{F_{0}}}\right\rangle \simeq(-0.2 \pm 2.4) 10^{-2}$

for our whole sample with the exception of the abovementioned ten stars of Sect. 13. No significant discrepancy is observed. It should be noted however that the five individual contributions to $\left(T_{\text {eff }}\right)_{\mathrm{CI}}$ may differ from each other, especially at high temperatures, according to

$\left(T_{\text {eff }}\right)_{\mathrm{CI}_{j}} \geq\left(T_{\mathrm{eff}}\right)_{\mathrm{CI}_{j+1}}$

for $j=1$ to 4 , the color indices being those indexed in Table 11. When making use of the values from Table 5, $V-[1.08]$ yields a grossly overestimated effective temperature for a HC-star while $J-K$ leads to an underestimate. This data should be adopted only when the five indices are available, using a mean of the estimates. We have tabulated the mean dereddened color indices as derived in

1 Stars not present in Stephenson's catalogue (1989) but showing some evidence of a $\mathrm{C} / \mathrm{O} \geq 1$ ratio.
Papers I to III, against the mean effective temperatures from Table 8 . This new calibration (Table 11) should be preferred since it is a second (better) iteration, and those indices may be used individually. As in the CV4-CV5 region (Sect. 6), we found a "shoulder" in the HC3-HC4 range, but here dispersions are larger and there is no observed angular diameter to rely on.

\section{The case of carbon stars with strong thermal emission}

\subsection{Introduction}

Part of our sample of carbon stars do exhibit substantial infrared excesses with respect to the photometric solution we adopted (see Papers II and III). This excess is interpreted in terms of thermal emission from circumstellar grains in a more or less spherical shell or flattened disc, or in even more complex structures (for instance CW Leo $=$ IRC+10216, see Paper III and references therein). The optical depth of those regions is often large. In addition, decoupling between circumstellar extinction and thermal emission occurs, as shown by the light curves observed at various wavelengths (see e.g. the case of RCB variables in Clayton 1996). Those Miras and/or "infrared" carbon stars, and RCB variables, are very few in our sample, predominantly of "optical" carbon stars. The visible photometry needed to apply our method, is frequently missing for such variables ("Miras"), while the latter ("RCBs") are intrinsically rare. Some peculiar stars with IR excesses, such as C3066 = HD 100764, are not found in these two categories.

The carbon stars with thick circumstellar shells are revealed here by anomalously high values of $C_{T_{\text {eff }}}$. For a cool carbon variable (CV6 or CV7), the consequence is

$\left(T_{\text {eff }}\right)_{F_{0}} \ll\left(T_{\text {eff }}\right)_{\mathrm{CI}}$,

the second value $\left(T_{\text {eff }}\right)_{\mathrm{CI}}$ being correct, provided dereddening has been performed and spectral bands contaminated by thermal emission not considered.

A different case is C3066 = HD 100764 and some $\mathrm{RCB}$ variables, both having been classified e.g. HC1 in our scheme (see Paper II). For those hot carbon stars, the high values of $C_{T_{\text {eff }}}$ lead to

$\left(T_{\text {eff }}\right)_{F_{0}} \gg\left(T_{\text {eff }}\right)_{\mathrm{CI}}$,

which is the opposite, but the correct estimate of the effective temperature is still the second one. Ten stars with obvious departures are documented in Table 12 .

The dereddening for the selective circumstellar + interstellar extinction is applied in the general case. Due account being taken of the IR excesses, a first estimate $m_{\text {bol1 }}$ of the apparent bolometric magnitude is obtained. This value takes into account the possible contribution of undetected neutral extinction, at least in the case of spherical symmetry. Conversely, the power subtracted by selective extinction may at least partly be re-radiated as thermal emission in the IR; an overestimate may occur. 
Table 12. Ten carbon stars with strong infrared excesses, either hot (HC) or cold (CV). The phase of the used photometric observations (SED), the derived photometric group and the color excess $E(B-V)$ are given, as well as the excess $E^{\prime}(B-V)$ as deduced from published maps of extinction (interstellar part, questionable values being denoted by colons). The effective temperature estimated here is $\left(T_{\text {eff }}\right)_{\mathrm{CI}}$ while $\left(T_{\text {eff }}\right)_{F_{0}}$ diverges (see text). Three estimates of the apparent bolometric magnitude are given (1: with dereddening and IR excess included, 2: with dereddening without IR excess, and 3: without dereddening with IR excess). The values finally adopted are quoted in the last column

\begin{tabular}{|c|c|c|c|c|c|c|c|c|c|c|c|}
\hline $\mathrm{C}$ & name & Phase & G & $E(B-V)$ & $E^{\prime}(B-V)$ & $\left(T_{\text {eff }}\right)_{\mathrm{CI}}$ & $\left(T_{\text {eff }}\right)_{F_{0}}$ & $m_{\text {bol 1 }}$ & $m_{\mathrm{bol} 2}$ & $m_{\mathrm{bol} 3}$ & $m_{\text {bol }}$ \\
\hline 2619 & CW Leo & 0.16 & CV7 & 2.34 & 0.0 & 1915 & 777 & 0.05 & 5.83 & 0.30 & 0.05 \\
\hline 2619 & CW Leo & 0.27 & CV7 & 3.67 & 0.0 & 2105 & 894 & 0.04 & 4.67 & 0.44 & 0.04 \\
\hline 2724 & RW LMi & 0.00 & CV6 & 0.88 & 0.0 & 2425 & 1305 & 2.75 & 5.98 & 2.89 & 2.75 \\
\hline 2724 & RW LMi & 0.25 & CV6 & 0.91 & 0.0 & 2470 & 1020 & 3.25 & 7.87 & 3.46 & 3.25 \\
\hline 3066 & HD 100764 & & $\mathrm{HC} 1$ & 0.00 & 0.0 & 4600 & 11670 & 7.87 & 8.51 & & 8.51 \\
\hline 3562 & S Aps & $\max$ & $\mathrm{HC} 1$ & 0.23 & 0.17 & 4510 & 5709 & 8.30 & 8.49 & & 8.30 \\
\hline 3950 & WX CrA & int & $\mathrm{HC} 1$ & 0.18 & 0.15 & 4805 & 8882 & 9.19 & 9.64 & & 9.64 \\
\hline \multirow[t]{5}{*}{4595} & V1468 Aql & & HC5 & 0.16 & 0.15 & 3455 & 2623 & 7.83 & 8.04 & & 8.04 \\
\hline & AFGL 799 & int & CV7 & 0.58 & 0.54 : & 1680 & 1514 & 6.69 & 8.45 & 6.80 & 6.69 \\
\hline & V688 Mon & 0.09 & CV7 & 2.23 & $0.7:$ & 1670 & 1432 & 5.25 & 7.31 & 5.57 & 5.25 \\
\hline & FX Ser & $\max$ & CV7 & 1.5 & 0.6 : & 2070 & 1842 & 4.45 & 5.02 & 4.77 & 4.45 \\
\hline & LP And & $\max$ & CV7 & 2.16 & 0.15 & 2040 & 1617 & 4.19 & 5.55 & 4.51 & 4.19 \\
\hline
\end{tabular}

It is worth noting however that Eq. (49) appears as nearly satisfied for more than 400 SEDs (see Table 10 at CDS).

\subsection{The hot carbon $(H C)$ stars}

The integrated fluxes and luminosities may well be overestimated if spherical geometry is not verified. The case of C3066 = HD 100764 is typical in this respect (see Paper II). No selective extinction was found $\left(A_{J} \simeq 0\right)$ and strong IR excesses start from the $H$-filter $(1.65 \mu \mathrm{m})$. The authors proposed a model of a disc inclined to the observer's line of sight. The IR excesses in this model do correspond to a power which was not initially directed toward the observer, but re-emitted and possibly partly scattered by dust in the disc. The value $\left(T_{\text {eff }}\right)_{\mathrm{CI}}=4600 \mathrm{~K}$ would correspond to $C_{T_{\text {eff }}} \simeq 2.363$ as compared to 3.153 from Eq. (37), the IR excess being included. Thus, if the model is adopted, the integrated flux should be corrected to

$F=1.78210^{-15}(2.363 / 3.153)^{2} \simeq 1.0010^{-15} \mathrm{Wcm}^{-2}(54)$

which is about $56 \%$ of the total observed flux. The apparent bolometric magnitude $m_{\text {bol1 }} \simeq 7.87$ then becomes

$m_{\mathrm{bol} 2} \simeq 7.87+0.63 \simeq 8.5$,

the temperature $\left(T_{\text {eff }}\right)_{\mathrm{CI}} \simeq 4600 \mathrm{~K}$ remaining. The magnitudes $m_{\text {bol2 }}$ quoted in Table 12 have been calculated from the dereddened SEDs extrapolated in the infrared (HC1SED), without including the excesses. An estimate of the latter can be obtained by comparison to the $m_{\text {bol1 }}$ values.

In the case of $\mathrm{C} 3950=\mathrm{WX} \mathrm{CrA}$, which is a RCB variable also classified $\mathrm{HC1}$, we obtained $E(B-V) \simeq 0.18$, compared to $E^{\prime}(B-V) \simeq 0.15-0.18$, the value for interstellar extinction as estimated from the published maps. The adopted dereddening is then based on 0.15 as a minimum (interstellar) value, the possible circumstellar contribution being less than 0.03. A strong excess is observed which starts from the $K$-filter $(2.2 \mu \mathrm{m})$. If this excess is caused by grains outside the line of sight, out of spherical symmetry, the corresponding flux should not be incorporated, exactly as advocated for C3066. This is the case of the "puffs" usually suggested in RCB-variables models. This is only in the less likely case of a neutral extinction with spherical symmetry, that the excess should be included as it is. The case of the RCB-variable $\mathrm{C} 3562=\mathrm{S}$ Aps classified HC1 with $E(B-V) \simeq 0.23$ and $E^{\prime}(B-V) \simeq 0.17$, is less clear with $E(B-V)_{\mathrm{CS}} \simeq$ 0.06 at maximum light. The carbon star C4595 = V1468 Aql with a silicate-type excess is classified HC5 with $E(B-V) \simeq 0.16$, compared to $E^{\prime}(B-V) \simeq 0.15$ from maps. The IR excess seems to develop from the $L$-filter $(3.6 \mu \mathrm{m})$. This excess should not be included in the calculation of the integrated flux. Finally, we adopt, in the four cases just studied,

$m_{\mathrm{bol}}=m_{\mathrm{bol} 2}$.

\subsection{The cool carbon (CV) variables}

We now consider the cases of 5 CV7 and 1 CV6 stars which exhibit both high selective extinctions and enormous IR excesses starting from the $1 \mu \mathrm{m}$ region. We might expect that part of the power is counted twice in $m_{\text {boll }}$, leading to an overestimate. The values of $m_{\mathrm{bol} 3}$ derived from the observed SED without dereddening (but including the IR excesses) are hardly different from those of $m_{\text {bol1 }}$. The IR excesses dominate the integrated fluxes, a circumstance confirmed except for FX Ser. The most extreme case in our sample is C2619 = CW Leo $=\mathrm{IRC}+10216$, classified as CV7. Its thermal emission represents the essential part of the total radiated flux. The high resolution IR images show the strongly non-spherical character of this emission (see Paper III and references therein). The interstellar contribution to the extinction is negligible $\left(E^{\prime}(B-V) \simeq 0\right)$ 


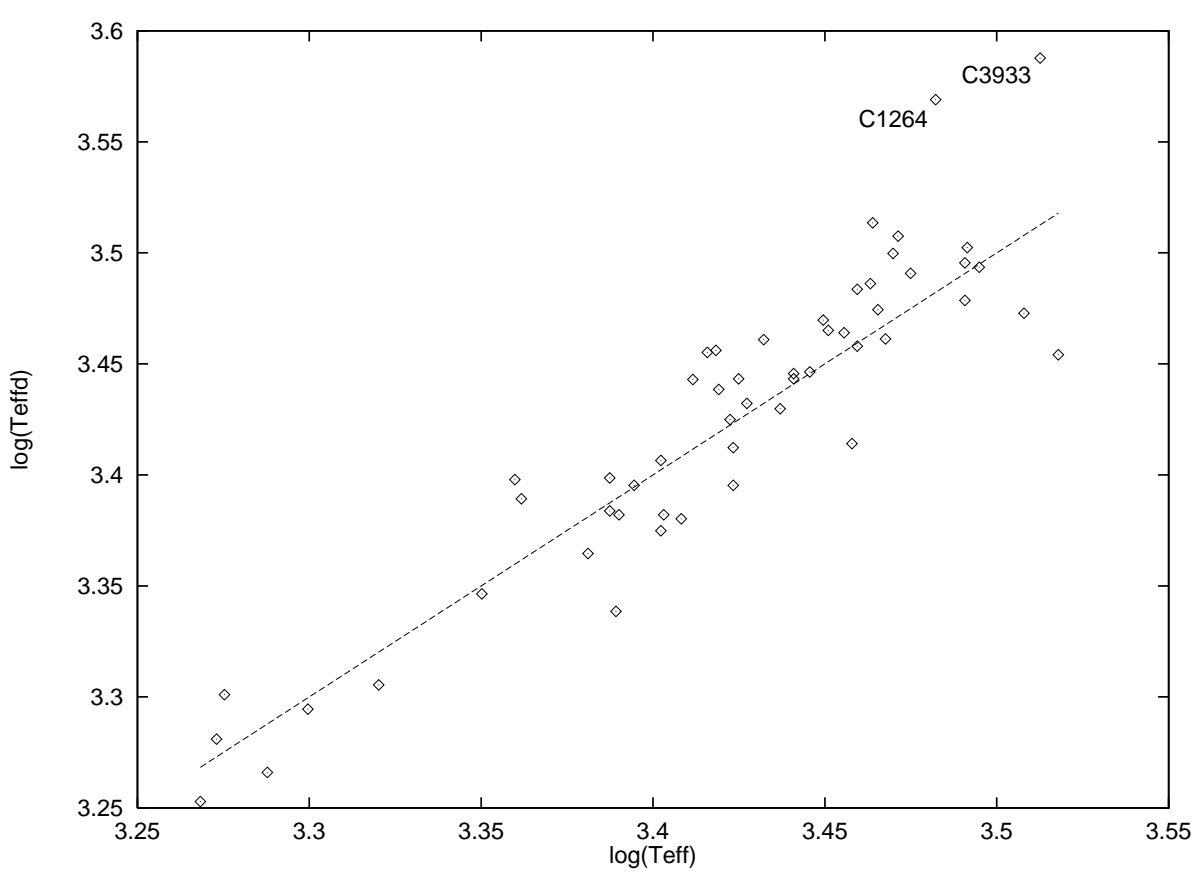

Fig. 12. The effective temperatures as directly deduced from 54 observed angular diameters (see Sect. 5) as a function of the finally adopted values taken from Table 10 or from Table A.1. The linear relation (58) is also shown as a dashed line (see text for details)

for this nearby star $(D \simeq 120 \mathrm{pc}$ according to Loup et al. $1993, \simeq 150 \mathrm{pc}$ in the authors' opinion). This is also the case of the more distant $\mathrm{C} 2724=\mathrm{RW} \mathrm{LMi}=\mathrm{CIT} 6$. If $D \simeq 150 \mathrm{pc}$ is adopted for $\mathrm{CW}$ Leo, the $m_{\text {bol2 }}$ values are an obvious underestimate since they yield $M_{\mathrm{bol}} \simeq 0.43$ and -0.72 which is quite unlikely. The $m_{\text {bol1 }}$ values lead to $M_{\text {bol }} \simeq-5.35$ at both phases, which is close to the values obtained for the other CV7 stars (Knapik et al. 2000). There remains the eventuality that those stars are seen in an "unfavorable" incidence, leading to an underestimated luminosity.

The remaining three objects with high selective extinctions (V688 Mon, FX Ser and LQ And), are affected by faint to moderate interstellar extinctions, according to published maps and roughly estimated distances. The object AFGL 799, classified CV7 with $E(B-V) \simeq 0.58$, should be set apart since its very uncertain interstellar contribution may amount to this value. Detailed nonspherical models and optical properties of the grains are needed to study the shell properties and stellar parameters more precisely. Finally, we adopt

$m_{\mathrm{bol}}=m_{\mathrm{bol} 1}$

for the six CV6 and CV7 stars of Table 12.

\subsection{Conclusion}

Grain emission is not restricted to the above ten stars.

They are the only stars amongst our sample for which $\left(T_{\text {eff }}\right)_{\mathrm{CI}}$ substantially differs from $\left(T_{\text {eff }}\right)_{F_{0}}$. Stars with smaller excesses were not considered in this section (e.g. $\mathrm{C} 234=\mathrm{R} \mathrm{Scl})$. The stars classified in an oxygen-rich photometric group were not discussed here since an only estimate of $T_{\text {eff }}$ is available for them, as obtained from published calibrations. This is the case of AC Her, a RV Tau-variable member of a binary around which a dust ring was observed at 11.8 and $18.7 \mu \mathrm{m}$ by Jura et al. (2000). The contribution of dust to atmospheric opacity and thermal emission is probably included in the intrinsic SEDs of many CV-stars, but the optical depths remain small. This is quite likely for most CV6-CV7 stars and only occasional in earlier CV-groups (e.g. R Scl was classified as CV4). The interested reader is referred to the discussions of Papers I to III. A few stars like the RV Tau-variable AC Her or the RCB-variables classified as oxygen-types, like R CrB or RY Sgr, should have their $m_{\text {bol1 }}$ values of Table 10 or Table A.1 corrected. They may need to be increased by a few tenths of a magnitude to reach a $m_{\text {bol2 }}$ level (lower luminosities).

\section{Comparison with the direct effective temperatures}

It is essential to check for the consistency of the final effective temperatures of Table 10 (or Table A.1) with the values directly deduced in Sect. 5 and quoted in Table 2. The values for individual stars may appreciably differ but they should be statistically consistent. In other words, since our calibrations ultimately rely on the measured angular diameters, we make sure that the procedures executed did not introduce any bias. The direct values $T_{\text {effd }}$ are plotted against the final values $T_{\text {eff }}$ on logarithmic scales, in Fig. 12. Two stars labelled in the diagram depart significantly from the locus of the fifty remaining ones. 

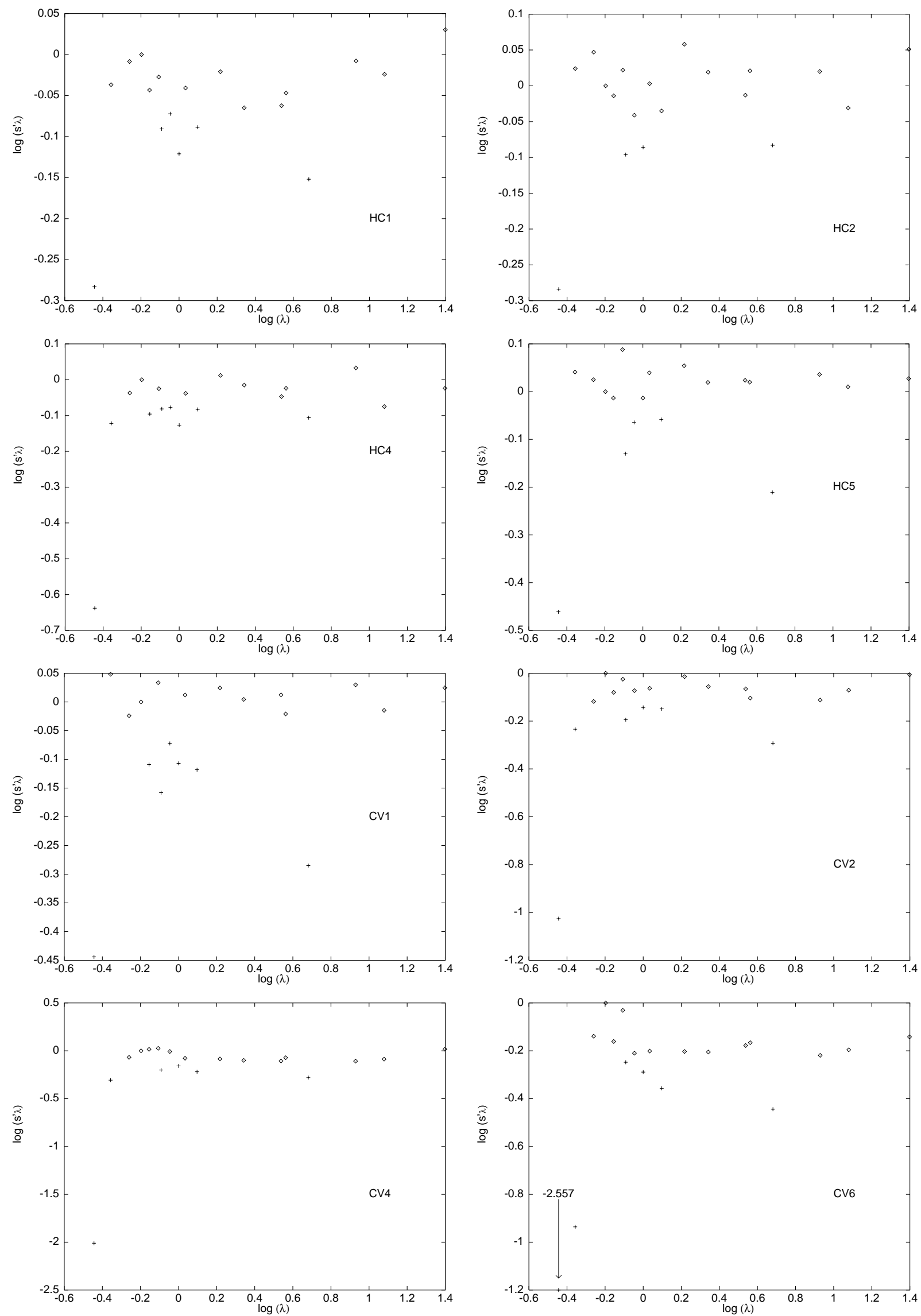

Fig. 13. The flux normalized to blackbody emission $s^{\prime}(\lambda)$ as defined in Sect. 15 for eight representative photometric groups. See text for the definition of "high" points (diamond-shaped symbols) and "low" points (crosses) 
An old inaccurate observation of C3933 = V4378 Sgr (see Table 2) was withdrawn from the statistics. The second observation, i.e. that of $\mathrm{C} 1264=\mathrm{BL}$ Ori, is more recent and accurate. Here again the direct effective temperature $(3700 \mathrm{~K})$ is much higher than usual for the photometric group obtained $(\mathrm{CV} 2$ and $E(B-V) \simeq 0)$. This solution is a very good one with consistent photometry at 16 wavelengths. The upper limit to extinction on the line of sight is $E^{\prime}(B-V) \leq 0.03$ as shown by the published maps (Burstein \& Heiles 1982). It is thus impossible to get a $\mathrm{HC} 4$ or HC5-group to reconcile this photometry with the effective temperature of $3700 \mathrm{~K}$, since $E(B-V) \simeq 0.6$ at least would be required. The spectral classification N0 and C6,3 is consistent with CV2 and $T_{\text {eff }} \simeq 3035 \mathrm{~K}$ we obtained, and not with $\mathrm{HC} 4$ or HC5. Variability is unlikely to explain this discrepancy. We conclude that either the measurement is less accurate than claimed, or that we exploit it wrongly in making use of Eq. (8). Finally, for the 52 remaining SEDs and diameters, we found

$\log T_{\text {effd }}=(1.003 \pm 0.056) \log T_{\text {eff }}-(0.009 \pm 0.024)$

with a correlation coefficient of 0.87 . No significant bias was introduced. The final scale is fully compatible with the direct values we started from.

\section{Comparison to the mean color temperatures}

Ridgway et al. (1981) showed that the effective temperatures of giant carbon stars are systematically higher than their mean color temperatures. In order to check this, mean color temperatures $T_{\mathrm{c}}$ were derived by trial and error. For any reference SED of a CV or HC-group, we minimized, as a function of wavelength, the variations of

$s^{\prime}(\lambda)=F_{\lambda} / \pi B_{\lambda}\left(T_{\mathrm{c}}\right)$

where $B_{\lambda}$ is the Planck function and $F_{\lambda}{ }^{\prime}$ s are the fluxes deduced from the unreddened color indices adopting $[1.08]=0$. Practically, this condition had to be relaxed in the UV in all cases, and in the blue and then in the visible, for more and more advanced CV-groups. This is the so-called blue-ultraviolet depression whose cause is still a matter of debate (e.g. Gilra 1973; Walker 1976; Bregman \& Bregman 1978; Orlati 1987; Johnson et al. 1988). The results are shown in Fig. 13.

Roughly speaking, one may consider two categories

- the "low" points correspond to photometric bands severely affected by one or several molecular bandheads or by a more or less continuous opacity increasing toward short wavelengths;

- the "high" points which are not much influenced, corresponding to either narrow bands without molecular bandheads ("continuum" points) or to bands wide enough with no strong bandheads.

It is possible to fit horizontal lines through the "high" points, i.e. to minimize the absolute value of the slope, the adopted blackbody temperature being the mean color temperature. The values thus obtained are quoted in Table 8 and shown in Fig. 14. It is found that the differences $\left\langle T_{\text {eff }}\right\rangle-T_{\mathrm{c}}$ are positive (Fig. 14), from $105 \mathrm{~K}$ (CV7) to $450 \mathrm{~K}$ (HC5). The only negative value is $-280 \mathrm{~K}$ for HC0, which should not be considered as significant.

\section{Comparison to the IRF method}

Tsuji (1981a, 1981b) applied the infrared flux method (IRFM) to carbon giants (Sect. 1). We consider here only the recent values published by Ohnaka \& Tsuji (1996, 34 stars in common), Aoki \& Tsuji (1997, 6 stars), and Ohnaka \& Tsuji (1999, 4 stars). Thus a total of 44 stars were found to be in common. The effective temperatures from the IRF method are plotted in Fig. 15, against the final BKR values from the present work (Table 10 or Table A.1). The first bisector is shown as a dashed line. An increasing discrepancy is observed for decreasing temperatures. The lowest temperature from IRFM is $2670 \mathrm{~K}$ in the present sample and $2420 \mathrm{~K}$ for our values.

We have obtained two linear fits from the method of least squares, that is for $\log T_{\text {eff }} \leq 3.522$ with $n=40$,

$$
\begin{array}{r}
\log \left(T_{\text {eff }}\right)_{\mathrm{IRFM}}=(0.449 \pm 0.083) \log \left(T_{\text {eff }}\right)_{\mathrm{BKR}} \\
+(1.929 \pm 0.019)
\end{array}
$$

and, for $\log T_{\text {eff }} \geq 3.477$ with $n=17$,

$\log \left(T_{\text {eff }}\right)_{\text {IRFM }}=(0.971 \pm 0.072) \log \left(T_{\text {eff }}\right)_{\text {BKR }}$

$$
+(0.100 \pm 0.023)
$$

respectively. The coefficients of determination are 0.43 and 0.92 respectively.

There is thus a good agreement for effective temperatures higher than $3170 \mathrm{~K}\left(\log T_{\text {eff }} \simeq 3.5\right)$ and a slope reduced to about 0.45 for temperatures lower than $3170 \mathrm{~K}$, that is a strong discrepancy between both methods. Specifically, Eq. (60) yields $\left(T_{\text {eff }}\right)_{\mathrm{IRFM}} \simeq 2808 \mathrm{~K}$ against $\left(T_{\text {eff }}\right)_{\mathrm{BKR}} \simeq 2420 \mathrm{~K}$ that is a departure of nearly $390 \mathrm{~K}$ at CV6. From Eq. (17) used at constant flux, the angular diameters should be reduced down to $74 \%$ of the previously adopted values $(-0.13 \mathrm{dex}$ in $\log )$, so as to shift our scale on the IRFM, which is well outside the error bars. A brightening on the discs producing $\eta$ as high as 1.81, would alternatively be required in Eq. (20).

Since our effective temperatures are consistent with the direct values from observed angular diameters (Sect. 14) and model predictions (Sects. 9 and 10), we conclude there is some difficulty in applying the IRFM to low temperatures. Ohnaka \& Tsuji (1996) reported ${ }^{12} \mathrm{C} /{ }^{13} \mathrm{C}$ ratios smaller (by a factor of 2 or 3 ) than the values of Lambert et al. (1986). This discrepancy is attributed by de Laverny \& Gustafsson (1998) to the effective temperatures adopted by Ohnaka \& Tsuji, which are higher than those of Lambert et al. by amounts of 260 to $450 \mathrm{~K}$.

\section{7. $\mathrm{C} / \mathrm{O}$ ratios and the $\mathrm{CV}$-classification}

The investigation of parameters for model atmospheres, such as relative extensions or masses and surface 


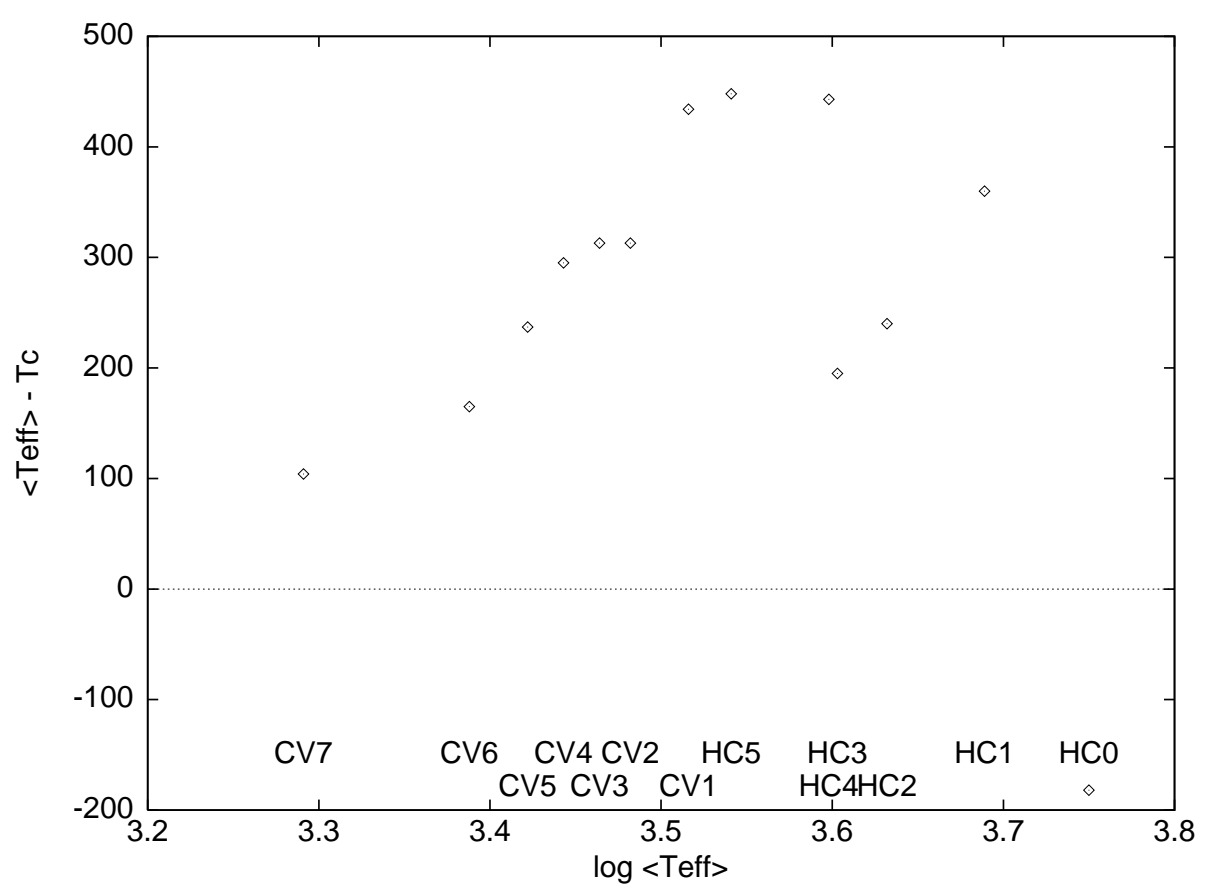

Fig. 14. Differences between mean effective temperatures and mean color temperatures along the sequence of groups from left to right (i.e. from $1900 \mathrm{~K}$ at $\mathrm{CV} 7$ to $5700 \mathrm{~K}$ at $\mathrm{HC} 0$ )

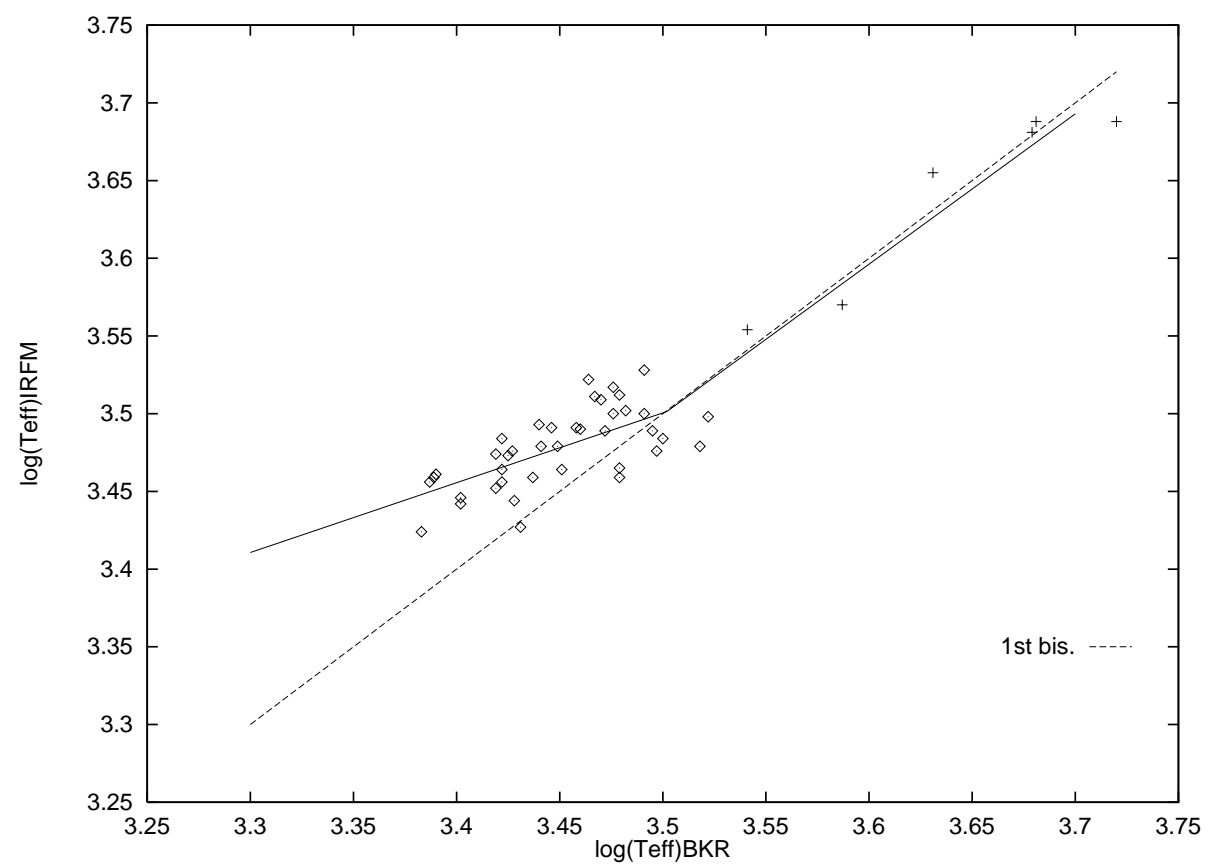

Fig. 15. Effective temperatures from IRFM as a function of effective temperatures on our new homogeneous scale. The dashed line is the first bisector. The two regression lines of Eqs. (60) and (61) are shown as continuous lines with a junction at $3170 \mathrm{~K}$

gravities $g$, is postponed to a forthcoming paper making use of the HIPPARCOS data (Knapik et al. 2000). It will be shown that $g$ is almost constant along the CVsequence. For the time being, we concentrate on $\mathrm{C} / \mathrm{O}$, an abundance ratio of prominent importance for model atmospheres but also for internal structure and evolution. The carbon overabundance in bright AGB stars is attributed to the "third dredge up" (TDU) phenomenon (Iben \& Renzini 1983; Lattanzio \& Boothroyd 1997), which happens when the convective envelope is able to penetrate the inter-shell region (between the $\mathrm{He}$ and $\mathrm{H}$ burning shells), i.e. during thermal pulses (TP-AGB stage). Looking for observational constraints, we search for a possible correlation between the $\mathrm{C} / \mathrm{O}$ ratios of $\mathrm{CV}$ stars and their classification or equivalently their effective temperatures.

Kilston (1975) published eight ratios derived from the curve of growth method. The values range from 1.03 to 


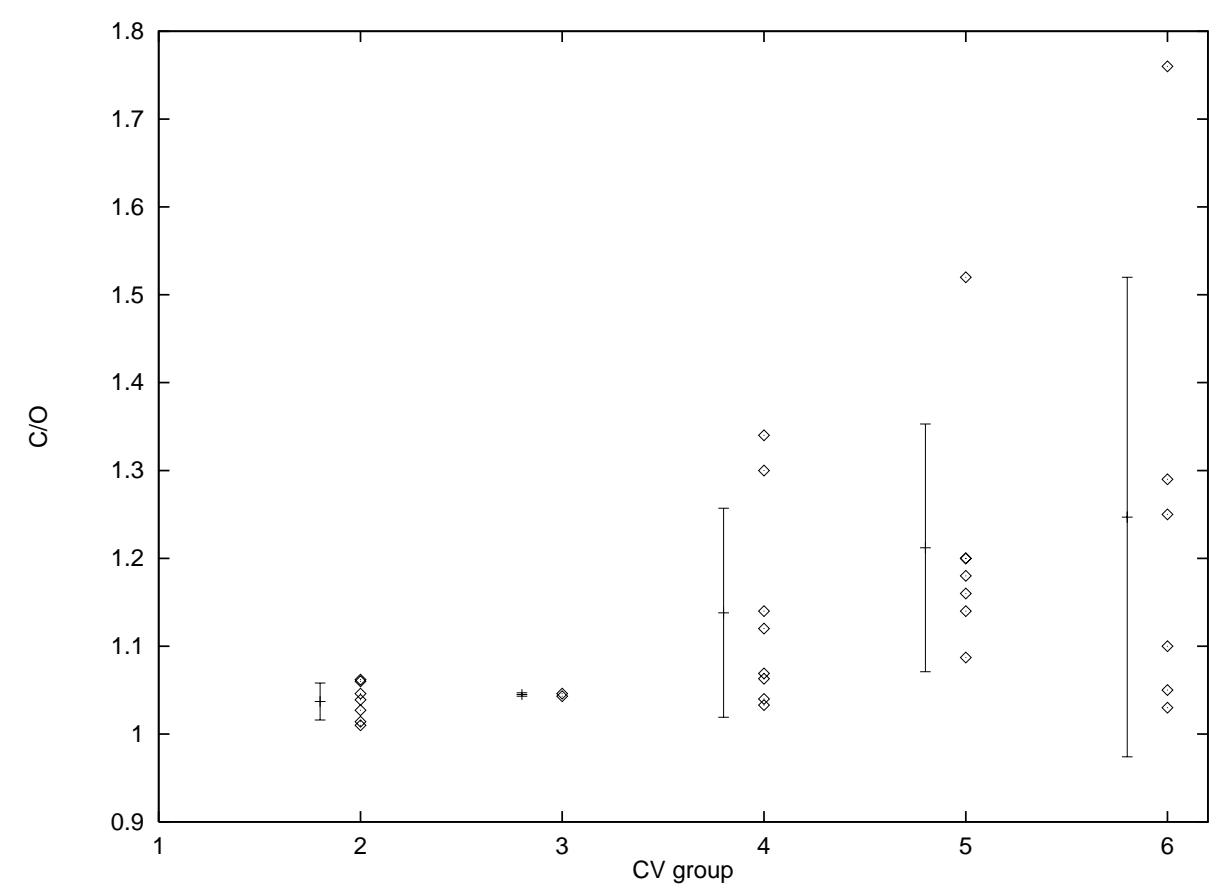

Fig. 16. The $\left({ }^{12} \mathrm{C}+{ }^{13} \mathrm{C}\right) /{ }^{16} \mathrm{O}$ ratio from Lambert et al. (1986) vs. the CV-group. The mean values and dispersions bars are also shown, slightly shifted to the left. Both increase with increasing CV-number, i.e. with decreasing effective temperature

2.9. More recent analyses with model atmospheres point to smaller values on average. Johnson et al. (1982) obtained for 2 stars ratios close to unity. We used the most extensive study available at present by Lambert et al. (1986), on $30 \mathrm{CV}$-stars. The $\left({ }^{12} \mathrm{C}+{ }^{13} \mathrm{C}\right) /{ }^{16} \mathrm{O}$ ratio is shown against our effective temperatures in Fig. 16 and Table 13, where mean values and dispersions are quoted. Unfortunately, Lambert et al. (1986) studied no CV1 or CV7 stars. It is remarkable that the largest ratio observed for CV2 and CV3 stars is only 1.062. Then, from CV4 to CV6, the maximum value, the mean value and thus the dispersion increase. The transition from low ratios to increasing ones probably occurs at $2800-2850 \mathrm{~K}$, or CV3-CV4. This value will be found again in Sect. 18 . We also note that $\mathrm{C} / \mathrm{O} \simeq$ 1.4 is usually adopted for CW Leo $=\mathrm{IRC}+10216$, the extreme CV7 object.

At this stage, one might suspect some defect in the models used in the spectral analysis, for instance some spurious influence of underestimated molecular band intensities. Similar behaviors, i.e. increases with decreasing effective temperatures, are observed for several molecular band intensities (e.g. $\mathrm{SiC}_{2}$, see Sect. 18) and emission from solid silicon carbide ( $\mathrm{SiC}$ ) as well (Bergeat 2000); all species whose abundances are directly connected to the carbon excess. The importance of mass loss and circumstellar emission also increases along the same sequence.

It can be seen from Fig. 16 that low $\mathrm{C} / \mathrm{O}$ ratios are widespread from $\mathrm{CV} 2$ to $\mathrm{CV} 6$. High $\mathrm{C} / \mathrm{O}$ ratios are absent from the early CV-groups. There is actually no univocal relation between both parameters, which are to be considered as independent.

Those results may be an insight into the evolution of cool carbon stars on the TP-AGB branch, especially in view of carbon dredging up (TDU). Additional parameters such as initial mass and initial chemical composition (metallicity) will be considered in Knapik et al. (2000).

\section{The $M-S$ band intensities and the effective temperatures}

As an illustration of the efficiency of the new effective temperature scale, we show here how it may help the understanding of molecular band intensities. Wallerstein \& Knapp (1998) raised doubts about the identification of $\mathrm{SiC}_{2}$ as the molecule responsible for the Merrill-Sanford (M-S) absorption bands observed near 490 and $497.7 \mathrm{~nm}$. The $\mathrm{SiC}_{2}$ molecule is usually mentioned to explain those bands which are either strong or absent. Intermediate intensities are noticed only in few spectra. Making use of the spectral types, Wallerstein \& Knapp argued that

- if a triatomic molecule is responsible, those M-S bands should get stronger and stronger in the coolest stars;

- among the 40 stars studied by Dominy (1985), 11 of 20 C4 and C5 stars show the M-S bands while only 5 of $17 \mathrm{C} 7-\mathrm{C} 9$ stars show them.

This doubt is entirely due to the spectral classification whose first index intended to be only temperature dependent, but is influenced by abundances. Many C7-C9 stars are actually relatively hot objects $\left(T_{\text {eff }} \geq 2900 \mathrm{~K}\right)$ found in groups $\mathrm{CV} 1$ to $\mathrm{CV} 3$, with $\mathrm{C} / \mathrm{O}$ ratios close to unity.

The intensities of the $\mathrm{M}-\mathrm{S}$ bands taken from Dominy (1985) range from 0 to 5. They are plotted in Fig. 17, against effective temperatures taken from Table 10 or Table A.1, for 37 stars in common. The mean value 


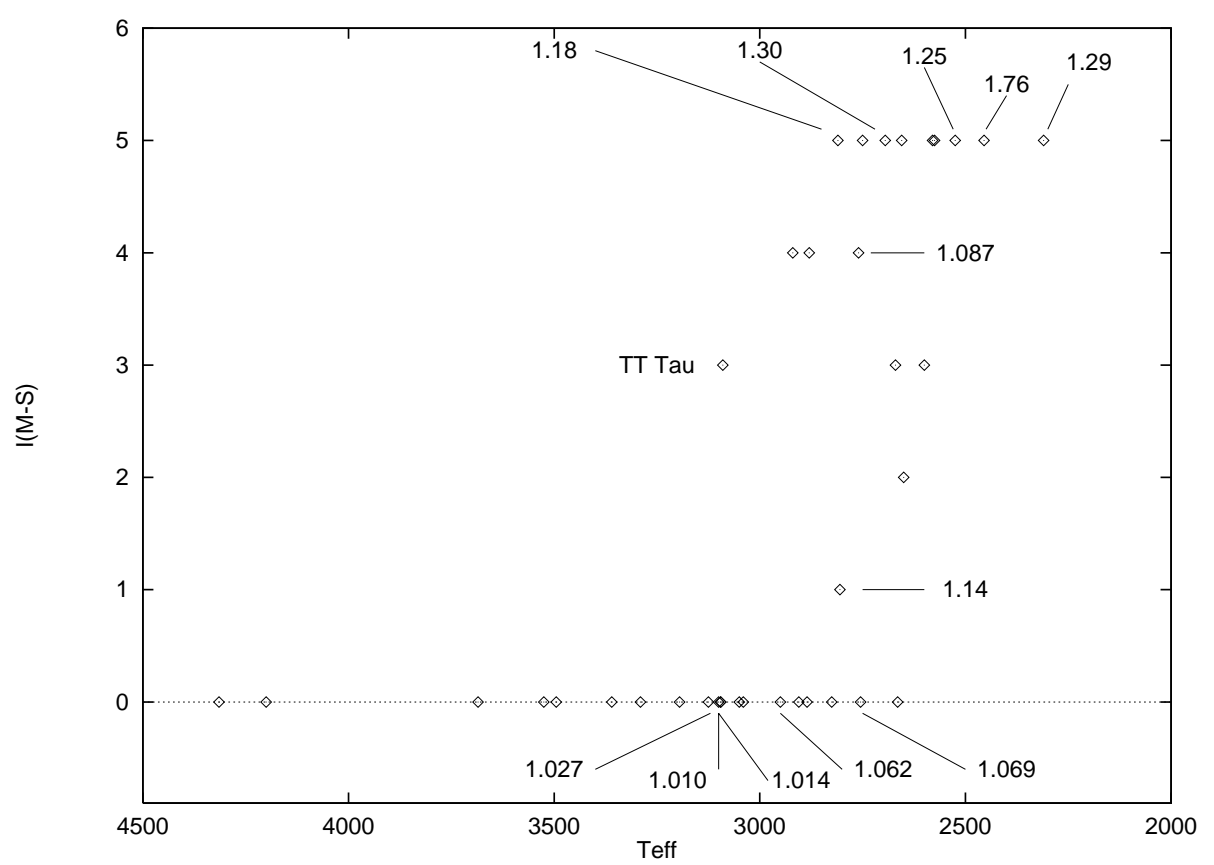

Fig. 17. The intensities of the Merrill-Sanford (M-S) bands from Dominy (1985) on a scale from 0 to 5, vs. the effective temperatures on our new scale. The available C/O ratios from Lambert et al. (1986) are mentioned as labels

$\left\langle T_{\text {eff }}\right\rangle \simeq 3040 \mathrm{~K}$ of its CV2 group was adopted for UX Cas which is absent from our tables. The $\mathrm{C} / \mathrm{O}$ ratios from Lambert et al. (1986) are shown as labels whenever they are available. We can see that, with the marginal exception of the labelled TT Tau, the transition from $I_{\mathrm{M}-\mathrm{S}}=0$ to 5 (maximum value) does occur in the $2650-2950 \mathrm{~K}$ range, approximately centered on the value $T_{\text {eff }} \simeq 2800 \mathrm{~K}$ already mentioned in Sect. 17 for a transition of C/O values. Lambert et al. (1986) concluded that their $\mathrm{C} / \mathrm{O}$ ratios clearly correlate with the $I_{\mathrm{M}-\mathrm{S}}$ intensities. The issue is no longer doubtful: effective temperatures and $\mathrm{C} / \mathrm{O}$ ratios contribute jointly. The hot atmospheres $\left(T_{\text {eff }} \geq 3000 \mathrm{~K}\right.$ with the possible exception of TT Tau) are free of $\mathrm{SiC}_{2}$ molecules. Near $T_{\text {eff }} \simeq(2800 \pm 150) \mathrm{K}$, the transition occurs for $1.07 \leq \mathrm{C} / \mathrm{O} \leq 1.17$ between "none" $(\langle\mathrm{C} / \mathrm{O}\rangle \simeq 1.04)$ and "strong" $(\langle\mathrm{C} / \mathrm{O}\rangle \simeq 1.36)$ for M-S bands. The CV4-CV6 stars with $T_{\text {eff }} \leq 2900 \mathrm{~K}$ are precisely those carbon stars which exhibit the strongest bands of $\mathrm{C}_{2}$ and $\mathrm{C}_{2} \mathrm{H}_{2}$. They also show the most prominent IR emission feature close to $\lambda \simeq 11.3 \mu \mathrm{m}$ (Bergeat $2000)$, which is attributed to $\mathrm{SiC}$ grains. The red system of the CN bands is a noticeable exception as evidenced by Baumert's data (1972). The new temperature scale and the classification in photometric groups, provide a sound basis in the study of those complex atmospheres.

\section{Conclusion}

We describe a method to determine the effective temperature of giant carbon stars. Angular diameters, spectrophotometric and photometric SEDs, and model atmospheres, were simultaneously used. A new homogeneous scale is proposed for the 1800 to $5800 \mathrm{~K}$ range. It is tightly connected to the classification in photometric groups and both data may be used for similar purposes. The effective temperature scale is calibrated against the 54 angular diameters observed so far during lunar occultations and/or from interferometry. The latter are consistent with uniform discs as the radiation sources, which nearly correspond to a Rosseland radius for Miras atmospheres with appreciable relative extensions. The scale obtained for effective temperatures is consistent with the predictions of model atmospheres over most of the abovementioned interval, except at the cool end where model improvements are required. The consistent use of angular diameters together with analyzed SEDs from photometry, was validated in Sect. 8 (Fig. 5), which is a central issue: the photometric quantity $\langle k\rangle^{1 / 2}$ was shown to be an angular diameter on a relative scale.

Finally, effective temperatures and apparent bolometric magnitudes are given for 438 SEDs of 410 carbon (and related) stars in the extensive Table 10 (only available at CDS) and in its condensed version (Table A.1). Among them, 24 SEDs are classified in oxygen-type groups (mainly the hottest RCBs) and 12 SEDs are in the SCVgroup. Effective temperatures and apparent bolometric magnitudes of stars with strong IR excesses were discussed separately. The main difficulties which remain are the lack of measured angular diameters on HC stars, and the poor adequacy of observations of very cool model atmospheres. Further versions of the hot and cool ends of the scale are to be expected once new observations and/or models have become available. 
Table 13. The effective temperatures of the $30 \mathrm{CV}$-types carbon stars with C/O ratios published by Lambert et al. (1986). The mean values of $\mathrm{C} / \mathrm{O}$ ratios and their dispersions are also given, which increase along the sequence of the CV-groups

\begin{tabular}{cccc}
\hline group & $\left\langle T_{\text {eff }}\right\rangle$ & $\langle\mathrm{C} / \mathrm{O}\rangle$ & $n$ \\
\hline CV2 & $3046 \pm 77$ & $1.037 \pm 0.021$ & 7 \\
CV3 & $2970 \pm 7:$ & $1.045 \pm 0.002:$ & 2 \\
CV4 & $2735 \pm 57$ & $1.138 \pm 0.119$ & 8 \\
CV5 & $2691 \pm 68$ & $1.212 \pm 0.141$ & 7 \\
CV6 & $2363 \pm 146$ & $1.247 \pm 0.273$ & 6 \\
\hline CV & $2740 \pm 250$ & $1.147 \pm 0.165$ & 30 \\
\hline
\end{tabular}

\begin{tabular}{|c|c|c|c|c|}
\hline $\mathrm{C}$ & name & $\mathrm{CV}$ & $T_{\text {eff }}$ & $\mathrm{C} / \mathrm{O}$ \\
\hline 36 & VX And & 6 & 2455 & 1.76 \\
\hline 198 & Z Psc & 2 & 3095 & 1.014 \\
\hline 234 & $\mathrm{R} \mathrm{Scl}$ & 4 & 2625 & 1.34 \\
\hline 540 & U Cam & 4 & 2695 & 1.30 \\
\hline 769 & ST Cam & 4 & 2805 & 1.14 \\
\hline 833 & R Lep & 6 & 2245 & 1.030 \\
\hline 853 & W Ori & 5 & 2625 & 1.16 \\
\hline 1042 & Y Tau & 4 & 2735 & 1.040 \\
\hline 1179 & TU Gem & 4 & 2715 & 1.12 \\
\hline 1264 & BL Ori & 2 & 3035 & 1.039 \\
\hline 1316 & UU Aur & 4 & 2760 & 1.063 \\
\hline 1565 & WCMa & 3 & 2975 & 1.046 \\
\hline 2378 & $\mathrm{X}$ Cnc & 5 & 2645 & 1.14 \\
\hline 2641 & Y Hya & 5 & 2645 & 1.52 \\
\hline 2803 & U Hya & 3 & 2965 & 1.043 \\
\hline 2835 & VYUma & 2 & 2930 & 1.060 \\
\hline 2877 & V Hya & 6 & 2160 & 1.050 \\
\hline 3283 & Y Cvn & 5 & 2760 & 1.087 \\
\hline 3313 & RY Dra & 5 & 2810 & 1.18 \\
\hline 4032 & TY Oph & 5 & 2680 & 1.20 \\
\hline 4038 & T Lyr & 6 & 2310 & 1.29 \\
\hline 4121 & S Sct & 4 & 2755 & 1.069 \\
\hline 4164 & V Aql & 6 & 2525 & 1.25 \\
\hline 4302 & UX Dra & 2 & 3090 & 1.046 \\
\hline 4333 & AQ Sgr & 4 & 2790 & 1.033 \\
\hline 4774 & RT Cap & 6 & 2480 & 1.10 \\
\hline 5418 & V460 Cyg & 2 & 2950 & 1.062 \\
\hline 5425 & RV Cyg & 5 & 2675 & 1.20 \\
\hline 5928 & TX Psc & 2 & 3125 & 1.027 \\
\hline 5976 & WZ Cas & 2 & 3095 & 1.010 \\
\hline
\end{tabular}

The result of Ridgway et al. (1981) is confirmed, that the effective temperatures of the carbon stars are systematically higher than the mean color temperatures, by a few hundred Kelvins. The effective temperatures from the infrared flux method (IRFM) are systematically higher than our values for $T_{\text {eff }} \leq 3000 \mathrm{~K}$. A good agreement is however observed for $T_{\text {eff }} \geq 3100 \mathrm{~K}$. Since the present scale is consistent with the 54 angular diameters available so far, there is probably some problem with the IRFM at low temperatures.
The HR diagram of cool giants with carbon-enriched atmospheres will be constructed in a forthcoming paper (Knapik et al. 2000), making use of the astrometric data from HIPPARCOS. Full discussions will then be developed. Herein, the impact of the classification into photometric groups and of the new effective temperature scale were illustrated. It was shown that the mean values, maximum values and thus dispersions of the $\mathrm{C} / \mathrm{O}$ ratio, increase along the sequence of the photometric $\mathrm{CV}$ groups, i.e. for decreasing effective temperatures. The intensities of the Merrill-Sanford (M-S) bands attributed to $\mathrm{SiC}_{2}$ increase abruptly with decreasing effective temperature and increasing $\mathrm{C} / \mathrm{O}$ ratios, from "none" to "strong" at $T_{\text {eff }} \simeq(2800 \pm 150) \mathrm{K}$ and $\mathrm{C} / \mathrm{O} \simeq 1.12 \pm 0.05$.

Note added in proof: New data becomes available which illustrates the variations of angular diameters of Miras with wavelength, in the near IR, and with phase (Thompson et al. to be submitted in the Astronomical Journal). In our Table 2, the previously published diameter $(2.28 \pm$ 0.65) mas adopted for C5265 = YY Eri should be replaced by the new $(2.65 \pm 0.03)$ mas leading to the new direct effective temperature $(2735 \pm 46) \mathrm{K}$, which is much more accurate. Additional angular diameters will become available for, at least, C500 = Y Per and C828= R Ori which are two carbon Miras.

Acknowledgements. Valuable suggestions from the referee Dr. Jan Martin Winters are gratefully acknowledged.

\section{References}

Aoki, W., \& Tsuji, T. 1997, A\&A, 317, 845

Aoki, W., Tsuji, T., \& Ohnaka, K. 1998, A\&A, 340, 222

Aoki, W., Tsuji, T., \& Ohnaka, K. 1999, A\&A, 350, 945

Avetysian, A. Kh., Zalinyan, V. P., Melik-Alaverdyan, Yu. K., Oganesyan, R. Kh., \& Tovmasyan, G. M. 1981, Astrofizika, 17, 225 (english version: Astrophysics 17, 118)

Baumert, J. H. 1972, The Ohio State university: unpublished thesis

Bergeat, J. 2000, in preparation

Bergeat, J., Sibille, F., Lunel, M., \& Lefèvre, J. 1976a, A\&A, 52,227

Bergeat, J., Lunel, M., Sibille, F., \& Lefèvre, J. 1976b, A\&A, 52,263

Bergeat, J., \& Knapik, A. 1997, A\&A, 321, L9

Bergeat, J., Knapik, A., \& Rutily, B. 1999, A\&A, 342, 773, Paper II

Blackwell, D. E., \& Shallis, M. J. 1977, MNRAS, 180, 177

Blackwell, D. E., Petford, A. D., \& Shallis, M. J. 1980, A\&A, 82,249

Blackwell, D. E., Petford, A. D., Haddock, D. J., \& Selby, M. J. 1990, A\&A, 232, 396

Blow, C. L., Chen, P. C., Edwards, D. A., Evans, D. S., \& Frueh, M. 1982, AJ, 87, 1511

Bouigue, R. 1954, Ann. Astrophys., 17, 104 
Bregman, J. D., \& Bregman, J. N. 1978, ApJ, 222, L41

Burnashov, V. I. 1979, Pub. Krimsk. Obs., 60, 32

Burstein, D., \& Heiles, C. 1982, AJ, 87, 1165

Cernicharo, J. 1998, Ap\&SS, 255, 303

Clayton, G. C. 1996, PASP, 108, 225

de Laverny, P., \& Gustafsson, B. 1998, A\&A, 332, 661

Dominy, J. F. 1985, PASP, 97, 1104

Draine, B. T., \& Lee, H. M. 1984, ApJ, 285, 89

Dyck, H. M., Benson, J. A., van Belle, G. T., \& Ridgway, S. T. 1996, AJ, 111, 1705

Dyck, H. M., van Belle, G. T., \& Benson, J. A. 1996, AJ, 112, 294

Eglitis, I. 1988, Nauchnye Informacii, 65, 103

Eglitis, I. 1990, Pub. Riga Obs., 68, 118

ESA, 1997, The HIPPARCOS Catalogue, ESA SP-1200 (ESA)

Fäy, T. D., \& Honeycutt, R. K. 1972, AJ, 77, 29

Fäy, T. D., Warren, W. H., Johnson, H. R., \& Honeycutt, R. K. 1974, AJ, 79, 634

Forrest, W. J., Houck, J. R., \& McCarthy, J. K. 1981, ApJ, 248,195

Gilra, D. P. 1973, in Interstellar Dust and Related Topics, IAU Symp. 52, ed. J. M. Greenberg, \& H. C. Van de Hulst (D. Reidel Publ. Co, Dordrecht), 517

Goebel, J. H., Bregman, J. D., Strecker, D. W., Witteborn, F. C., \& Erikson, E. F. 1978, ApJ, 222, L129

Goebel, J. H., Bregman, J. D., Goorvitch, D., et al. 1980, ApJ, 235,104

Goebel, J. H., Bregman, J. D., Witteborn, F. C., Taylor, B. J., \& Willner, S. P. 1981, ApJ, 246, 455

Goebel, J. H., Goorvitch, D., Lanrson, H. P., \& Alexander, D. R. 1993, ApJ, 402, 680

Gunn, J. E., \& Stryker, L. L. 1983, ApJS, 52, 121

Houdashelt, M. L., Bell, R. A., Sweigart, A. V., \& Wing, R. F. 2000, AJ, 119, 1424

Iben, I., \& Renzini, A. 1983, ARA\&A, 21, 27

IRAS Science Team 1986, A\&AS, 65, 607 (LRS)

IRAS Point Source Catalog, version 2 1988, Joint IRAS Science Working Group, vols. 1-6, NASA RP-1190, Washington, DC: U.S. Government Printing Office

Jacob, A. P., Bedding, T. R., Robertson, J. G., \& Scholtz, M. 2000, MNRAS, 312, 733

Johnson, H. L., \& Méndez, M. E. 1970, AJ, 75, 785

Johnson, H. L., Obrien, G. T., \& Climenhaga, J. L. 1982, ApJ, 254,175

Johnson, H. L., Alexander, D. R., Bower, C. D., et al. 1985, ApJ, 292, 228

Johnson, H. R., Baumert, J. H., Querci, F., \& Querci, M. 1986, ApJ, 311, 960

Johnson, H. R., \& Luttermoser, D. G. 1987, ApJ, 314, 329

Johnson, H. R., Luttermoser, D. G., \& Faulkner, D. R. 1988, ApJ, 332, 421

Johnson, H. R., Ensman, L. M., \& Alexander, D. R., et al. 1995, ApJ, 443, 281

Jorgensen, U. G. 1989, ApJ, 344, 901

Jorgensen, U. G., Johnson, H. R., \& Nordlund, A. 1996, A\&A, 261,263

Jura, M., Chan, C., \& Werner, M. W. 2000, ApJ, 541, 264

Kholopov, P. N., Samus, N. N., Frolov, M. S., et al. 1985, General Catalogue of Variable Stars, Nauka Publishing House, Moscow (GCVS), Suppl. Lists 67 (1985, IBVS 2681), 68 (1987, IBVS 3058), 69 (1989, IBVS 3323), 70 (1990, IBVS 3530), 71 (1993, IBVS 3840), 72 (1995, IBVS 4140), 73 (1997, IBVS 4471), 74 (1999, IBVS 4659), \& 75 (2000, IBVS 4870)
Kilston, S. 1975, PASP, 87, 189

Knapik, A. 1999, Thèse, Université Claude Bernard - Lyon 1

Knapik, A., \& Bergeat, J. 1997, A\&A, 321, 236, Paper I

Knapik, A., Bergeat, J., \& Rutily, B. 1998, A\&A, 334, 545

Knapik, A., Bergeat, J., \& Rutily, B. 1999, A\&A, 344, 263, Paper III

Knapik, A., Bergeat, J., \& Rutily, B. 2000, submitted to A\&A

Lambert, D. L., Gustafsson, B., Eriksson, K., \& Hinkle, K. H. 1986, ApJS, 62, 373

Lattanzio, J. C., \& Boothroyd, A. I. 1997, in AIP Conference Proc. 402, ed. T. Bernatowitz, \& E. Zimer, AIP: Woodbury, NY

Lázaro, C., Hammersley, P. L., Clegg, R. E. S., et al. 1994, MNRAS, 269, 365

Loup, C., Forveille, T., Omont, A., \& Paul, J. F. 1993, A\&AS, 99, 291

Mathis, J. S. 1990, ARA\&A, 28, 37

McKellar, A., \& Buscombe, W. 1948, Pub. of Domin. Astrophys. Obs., 24, 361

Mendoza, E. E., \& Johnson, H. L. 1965, ApJ, 141, 161

Merrill, K. M., \& Stein, W. A. 1976, PASP, 88, 285

Noguchi, K., Maihara, T., Okuda, H., Sato, S., \& Mukai, T. 1977, PASJ, 29, 511

Oganesyan, R. Kh., Nersisyan, S. E., \& Karapetyan, M. Sh. 1985, Astrofizika, 23, 99 (english version: 1986, Astrophysics, 22, 432)

Ohnaka, K., \& Tsuji, T. 1996, A\&A, 310, 933

Ohnaka, K., \& Tsuji, T. 1999, A\&A, 345, 233

Orlati, M. A. 1987, ApJ, 317, 819

Perrin, G., Coudé du Foresto, V., Ridgway, S. T., \& Mariotti, J.-M. 1998, A\&A, 331, 619

Pollack, J. B., Toon, O. B., \& Khare, B. N. 1973, Icarus, 19, 372

Querci, F., Querci, M., \& Tsuji, T. 1974, A\&A, 31, 265

Querci, F., \& Querci, M. 1976, A\&A, 49, 443

Quirrenbach, A., Mozurkewich, D., Hummel, C. A., Buscher, D. F., \& Armstrong, J. T. 1994, A\&A, 285, 541

Richichi, A., Chandrasekhar, T., Lisi, F., et al. 1995, A\&A, 301, 439

Richichi, A., Stecklum, B., Herbst, T. M., \& Lagage, P. O. 1998a, A\&A, 334, 585

Richichi, A., Ragland, S., Stecklum, B., \& Leinert, C. H. 1998b, A\&A, 338, 527

Richichi, A., Fabbroni, L., Ragland, S., \& Scholz, M. 1999, A\&A, 344, 511

Ridgway, S. T., Wells, D. C., \& Joyce, R. R. 1977, AJ, 82, 414

Ridgway, S. T., Jacoby, G. H., \& Wells, D. C. 1980, AJ, 85, 1496

Ridgway, S. T., Jacoby, G. H., Joyce, R. R., \& Wells, D. C. 1981, in Physical Processes in Red Giants, ed. I. Iben Jr., \& A. Renzini (D. Reidel Publishing Company), 47

Ridgway, S. T., Jacoby, G. H., Joyce, R. R., Siegel, M. J., \& Wells, D. C. 1982, AJ, 87, 808

Schmidtke, P. C., Africano, J. L., Jacoby, J. H., Joyce, R. R., \& Ridgway, S. T. 1986, AJ, 91, 961

Scholz, M., \& Takeda, Y. 1987, A\&A, 186, 200

Stephenson, C. B. 1973, Pub. of the Warner \& Swasey Obs. 1, No. 4

Stephenson, C. B. 1976, Pub. of the Warner \& Swasey Obs. 2, No. 2

Stephenson, C. B. 1989, Pub. of the Warner \& Swasey Obs. 3, No. 2

Tsuji, T. 1981a, JA\&A, 2, 95 
Tsuji, T. 1981b, JA\&A, 2, 253

van Belle, G. T., Dyck, H. M., Benson, J. A., \& Lacasse, M. G. 1996, AJ, 112, 2147 van Belle, G. T., Dyck, H. M., Thompson, R. R., Benson, J. A., \& Kannappan, S. J. 1997, AJ, 114, 2150 van Belle, G. T., \& Thompson, R. R. 1999, BAAS, 31, 1435 Vanture, A. D. 1992, AJ, 104, 1997

Volk, K. \& Cohen, M. 1989, AJ, 93, 931

Walker, A. R. 1976, MNRAS, 174, 609
Walker, A. R., Wild, P. A. T., \& Byrne, P. B. 1979, MNRAS, 189, 455

Wallerstein, G., \& Knapp, G. R. 1998, ARA\&A, 36, 369

White, N. M., \& Feiermann, B. H. 1987, AJ, 94, 781

Wilson, L. A. 1986, in Late Stages of Stellar Evolution, S. Kwok, \& S. R. Pottasch (Reidel publishing Co., Dordrecht, Holland)

Wyller, A. A. 1960, Astrophysica Norvegica VII, No. 2, 13 
Table A.1. Short version of Table 10 only available at CDS (Strasbourg). The stars are identified by their C-entries (Col. 1) in Stephenson's catalogues (1989), or S (1976). Their names are given in the second column (Kholopov et al. 1985) and additional lists for variables stars, HD or BD or CD, IRAS 1988 as IR, and HIC for the INCA catalogue). The quoted data is the effective temperature on the new scale (Col. 3) and the apparent bolometric magnitude after dereddening, with possible IR excesses included (Col. 4). Whenever available, the phases in the variation cycles are given in Col. 5

\begin{tabular}{|c|c|c|c|c|c|c|c|c|c|c|c|c|c|c|}
\hline Ent. & Name & $T_{\text {eff }}$ & $m_{\text {bol1 }}$ & $\mathrm{Ph}$. & Ent. & Name & $T_{\text {eff }}$ & $m_{\text {bol1 }}$ & $\mathrm{Ph}$. & Ent. & Name & $T_{\text {eff }}$ & $m_{\text {bol1 }}$ & $\mathrm{Ph}$. \\
\hline 32 & ST Cas & 3500 & 6.50 & & 36 & VX And & 2455 & 4.27 & & 53 & NQ Cas & 3685 & 6.34 & \\
\hline 65 & AQ And & 2660 & 4.75 & & 80 & $\mathrm{BD}+21^{\circ} 64$ & 4180 & 9.13 & & 135 & $\mathrm{BD}+22^{\circ} 123$ & 4510 & 7.51 & \\
\hline 136 & W Cas & 3050 & 5.46 & & 196 & HD 7526 & 4270 & 9.37 & & 198 & Z Psc & 3095 & 3.83 & \\
\hline 234 & $\mathrm{R} \mathrm{Scl}$ & 2625 & $2.90^{\mathrm{a}}$ & $\max :$ & 238 & WW Cas & 2750 & 5.53 & & 256 & $\mathrm{CD}-19^{\circ} 290$ & 4005 & 10.20 & \\
\hline 258 & HD 10386 & 3960 & 8.12 & & 268 & V 547 Per & 4090 & 7.06 & & 295 & X Cas & 2445 & 5.58 & \\
\hline 327 & V Ari & 3475 & 6.79 & & 350 & BS Per & 2670 & 5.77 & & 357 & NSV 835 & 3940 & 8.46 & \\
\hline 361 & R For & 2060 & 3.86 & $\max$ & 378 & HD 16115 & 4240 & 7.54 & & 384 & VZ Per & 3320 & 6.98 & \\
\hline 387 & UY And & 3220 & 7.35 & & 451 & V 623 Cas & 3360 & 4.07 & & 461 & V 410 Per & 2885 & 5.53 & \\
\hline 471 & TW Hor & 2950 & 3.15 & & 496 & V 384 Per & 1820 & 3.86 & 0.97 & 500 & Y Per & 3525 & 5.85 & 0.98 \\
\hline 540 & U Cam & 2695 & 3.51 & & 541 & V 466 Per & 2575 & 4.23 & & 556 & AC Per & 2665 & 5.35 & \\
\hline 576 & HD 24281 & 4050 & 7.21 & & 588 & NSV 1426 & 4175 & 8.75 & & 594 & $\mathrm{BD}+23^{\circ} 601$ & 4010: & 9.60 & \\
\hline 608 & UV Cam & 3495 & 4.96 & & 610 & HIC 19050 & 4185 & 9.46 & & 623 & FR Ser & 2885 & 6.32 & \\
\hline 639 & HD 26667 & 4785 & 9.14 & & 643 & SY Per & 2705 & 4.92 & & 714 & V $718 \mathrm{Tau}$ & 1855 & 6.55 & 0.54 \\
\hline 725 & HD 29154 & 4795 & 8.28 & & 769 & ST Cam & 2805 & 3.44 & & 781 & HD 30443 & 4115 & 5.60 & \\
\hline 788 & T Cae & 3030 & 5.32 & & 793 & V 1060 Tau & 2925 & 5.69 & & 794 & TT Tau & 3090 & 3.96 & \\
\hline 797 & V 346 Aur & 2880 & 4.74 & & 806 & AU Aur & 2665 & 5.83 & & 828 & R Ori & 3520 & 6.79 & 0.79 \\
\hline 828 & R Ori & 2645 & 7.43 & 0.58 & 833 & R Lep & 2290 & 2.77 & 0.08 & 833 & R Lep & 2245 & $3.10^{\mathrm{a}}$ & 0.38 \\
\hline 836 & EL Aur & 2730 & 4.46 & & 853 & W Ori & 2625 & 2.87 & & 860 & TX Aur & 2835 & 5.34 & \\
\hline 875 & SY Eri & 2885 & 5.38 & & 893 & V 431 Ori & 2540 & 4.89 & & 904 & V 348 Aur & 2880 & 5.64 & \\
\hline 911 & UV Aur & 2920 & 4.97 & 0.07 & 911 & UV Aur & 2840 & $5.39^{\mathrm{a}}$ & 0.64 & 914 & V 1368 Ori & 3020 & 4.95 & \\
\hline 941 & S Aur & 1940 & 4.84 & 0.5 & 950 & CM Aur & 3285 & 6.63 & & 958 & GS Ori & 2980 & 7.30 & \\
\hline 972 & OV Aur & 2900 & 7.52 & & 984 & HD $36598^{\mathrm{b}}$ & 4570 & 7.44 & & 988 & RT Ori & 2870 & 4.83 & \\
\hline 998 & S Cam & 2775 & 5.94 & & 1004 & SZ Lep & 3215 & 5.69 & & 1006 & IR05352+2247 & 2300 & 6.31 & \\
\hline 1035 & $\mathrm{BD}-16^{\circ} 1217$ & 4815 & 9.59 & & 1038 & TU Tau & 2850 & 4.31 & & 1038 & TU Tau & 2855 & 4.64 & \\
\hline 1042 & Y Tau & 2735 & 3.33 & & 1043 & CP Tau & 2960 & 6.35 & & 1052 & W Pic & 2530 & 4.27 & \\
\hline 1061 & FU Aur & 3035 & 5.00 & & 1110 & $\mathrm{BD}+33^{\circ} 1194$ & 4850 & 9.20 & & 1128 & AZ Aur & 3190 & 4.91 & 0.09 \\
\hline 1128 & AZ Aur & 2310 & 5.79 & 0.51 & 1179 & TU Gem & 2715 & 3.90 & & 1187 & IR06088+1909 & 2015 & 7.48 & $\max$ \\
\hline 1190 & EI Ori & 2415 & 7.53 & & 1222 & GK Ori & 2430 & 5.47 & & 1226 & V 1393 Ori & 2900 & 6.19 & \\
\hline 1244 & V Aur & 2820 & 5.76 & & 1246 & BN Mon & 2410 & 5.49 & int & 1251 & ZZ Gem & 2530 & 6.27 & 0.13 \\
\hline 1256 & V 720 Mon & 3020 & 6.39 & & 1263 & IV CMa & 2690 & 5.16 & $\max$ & 1263 & IV CMa & 2655 & 5.40 & int \\
\hline 1264 & BL Ori & 3035 & 3.74 & & 1269 & AB Gem & 2450 & 5.77 & $\max$ & 1292 & HX Gem & 2910 & 6.75 & \\
\hline 1300 & RV Aur & 2920 & 5.97 & & 1309 & CR Gem & 2960 & 4.22 & & 1316 & UU Aur & 2760 & 2.32 & \\
\hline 1332 & IR06347-1203 & 2635 & 5.98 & & 1337 & NY Gem & 2445 & 7.02 & & 1355 & VW Gem & 3000 & 5.46 & $\max$ \\
\hline 1355 & VW Gem & 2985 & 5.64 & int & 1373 & V738 Mon & 2910 & 5.93 & & 1378 & CZ Mon & 2640 & 5.91 & \\
\hline 1380 & HD 48773 & 4225 & 8.64 & & 1392 & GO CMa & 2850 & 6.19 & & 1401 & DF Mon & 2450 & 6.20 & \\
\hline 1444 & W Mon & 2875 & 6.04 & & 1453 & GY Mon & 3060 & 5.26 & & 1460 & KY CMa & 4300: & 8.12 & \\
\hline 1466 & UW Aur & 3265 & 6.91 & & 1474 & BG Mon & 2975 & 6.96 & & 1478 & NP Pup & 3090 & 3.99 & \\
\hline 1489 & RV Mon & 2910 & 4.43 & & 1507 & V 614 Mon & 3320 & 4.95 & & 1549 & RY Mon & 2440 & 4.28 & \\
\hline 1561 & R CMi & 3690 & 4.68 & 0.12 & 1561 & R CMi & 3230 & 5.65 & 0.90 & 1561 & R CMi & 3160 & 5.43 & 0.31 \\
\hline 1565 & W CMa & 2960 & 4.05 & & 1565 & W CMa & 2990 & 4.04 & & 1595 & VX Gem & 2880 & 6.03 & \\
\hline 1615 & MO CMa & 2800 & 6.80 & & 1616 & $\mathrm{BK} \mathrm{CMi}$ & 2620 & 5.98 & & 1622 & RU Cam & 5215 & 8.09 & \\
\hline 1653 & BM Gem & 3295 & $5.97^{\mathrm{c}}$ & & 1659 & MY CMa & 2825 & 7.51 & & 1686 & V 578 Mon & 3060 & 6.45 & \\
\hline 1695 & $\mathrm{BE} \mathrm{CMa}$ & 2960 & 6.21 & & 1703 & HD 58364 & 3860: & 8.08 & & 1704 & V 760 Mon & 3340 & 6.19 & \\
\hline 1732 & NSV 3610 & 2040 & 4.77 & $\max$ & 1732 & NSV 3610 & 2055 & 5.34 & int & 1732 & NSV 3610 & 1815 & 5.28 & $\min$ \\
\hline 1737 & NQ Gem & 3440 & 5.98 & & 1787 & $\mathrm{BE} \mathrm{CMi}$ & 2905 & 5.86 & & 1790 & HD 60952 & 3410 & 7.42 & \\
\hline 1813 & V 765 Mon & 2450 & 9.56 & & 1819 & NSV 3676 & 2435 & 6.53 & & 1877 & GO Pup & 2415 & 6.23 & \\
\hline 1881 & W CMi & 3020 & 6.52 & & 1891 & V 767 Mon & 2705 & 6.99 & & 1910 & QT Pup & 2970 & 6.14 & \\
\hline 1941 & IR07525-3213 & 2595 & 6.82 & & 1944 & V 768 Mon & 2950 & 7.66 & & 1950 & IR07528-4346 & 2915 & 7.68 & \\
\hline 1968 & V 406 Pup & 2875 & 4.77 & & 1981 & HIC 39118 & $3655:$ & 9.64 & & 2007 & IR08002-0159 & 2915 & 6.44 & \\
\hline 2011 & IR08002-3803 & 3015 & 7.06 & & 2024 & IK Pup & 2675 & 7.06 & & 2033 & $\mathrm{CD}-30^{\circ} 5440$ & 3855 & 7.87 & \\
\hline 2051 & RT Pup & 3330 & 5.62 & & 2063 & IR08050-2939 & 2515 & 6.87 & & 2064 & RU Pup & 2680 & 4.96 & \\
\hline 2101 & V 346 Pup & 1875 & 4.82 & int & 2150 & RY Hya & 2440 & 5.33 & & 2153 & V 433 Pup & 3380 & 7.35 & \\
\hline 2156 & MT Hya & 3270 & 7.35 & & 2165 & T Lyn & 2650 & 6.10 & & 2177 & AC Pup & 2675 & 5.91 & \\
\hline 2219 & YY Pyx & 2835 & 6.34 & & 2247 & IR08313-2946 & 3040 & 7.60 & & 2272 & NV Vel & 2935 & 6.17 & \\
\hline 2282 & $\mathrm{BD}+75^{\circ} 348$ & 5075 & 9.21 & & 2301 & HIC 42672 & 4065 & 7.65 & & 2315 & GV Vel & 2465 & 6.99 & \\
\hline 2326 & R Pyx & 2440 & 5.83 & & 2331 & UZ Pyx & 3325 & 4.93 & $\max$ & 2334 & UW Pyx & 2040 & 6.30 & \\
\hline 2378 & $\mathrm{X}$ Cnc & 2645 & 3.49 & & 2383 & HD 76396 & 4980 & 8.54 & & 2384 & T Cnc & 2405 & 4.29 & 0.1 \\
\hline 2384 & $\mathrm{~T}$ Cnc & 2525 & $4.08^{\mathrm{a}}$ & 0.7 & 2396 & HD 78646 & 4640: & 8.83 & & 2404 & DH UMa & 3500 & 7.62 & \\
\hline 2428 & HD 78278 & 3940 & 9.85 & & 2449 & GM Cnc & 3485 & 6.90 & & 2450 & IQ Hya & 2520 & 5.63 & \\
\hline 2463 & RU Car & 2990 & 6.99 & & 2619 & CW Leo & 1915: & $0.05^{\mathrm{a}}$ & 0.16 & 2619 & CW Leo & 2105: & $0.04^{\mathrm{a}}$ & 0.27 \\
\hline 2626 & HD 85066 & 4000 & 8.74 & & 2635 & W Sex & 3305 & 6.64 & & 2641 & Y Hya & 2645 & 3.54 & \\
\hline 2656 & FP Vel & 3015 & 6.86 & & 2661 & X Vel & 2700 & 3.51 & & 2685 & SZ Car & 2810 & 4.60 & \\
\hline 2713 & AB Ant & 3030 & 4.41 & & 2715 & HD 88627 & 4260 & 9.43 & & 2724 & RW LMi & 2425: & $2.75^{\mathrm{a}}$ & 0.0 \\
\hline 2724 & RW LMi & 2470: & $3.25^{\mathrm{a}}$ & 0.25 & 2738 & XZ Vel & 2430 & 4.36 & $\max$ & 2759 & HD 90935 & 4085: & 7.27 & \\
\hline 2764 & CZ Hya & 2525 & 5.16 & 0.6 & 2790 & TV Vel & 3250 & 6.30 & 0.7 & 2793 & U Ant & 2810 & 2.58 & \\
\hline 2803 & U Hya & 2965 & 2.30 & & 2829 & HD $92626^{b}$ & 4290 & 6.59 & & 2835 & VY UMa & 2930 & 3.57 & \\
\hline 2852 & TZ Car & 3265 & 5.28 & & 2877 & V Hya & 2160 & 2.60 & & 2885 & SS Vel & 2810 & 6.31 & \\
\hline 2892 & $\mathrm{BD}-18^{\circ} 3055$ & 4000: & 9.84 & & 2900 & $\mathrm{BD}+16^{\circ} 2188$ & 4195 & 9.63 & & 2914 & $\mathrm{BD}+42^{\circ} 2173$ & 4330 & 9.54 & \\
\hline 2919 & $\mathrm{BD}+41^{\circ} 2150$ & 4120 & 9.80 & & 2959 & RW Cen & 2950 & 5.64 & & 2967 & CI Cha & 2860 & 5.39 & \\
\hline 2975 & HD 97578 & 5080 & 8.33 & & 2984 & SY Car & 3455 & 6.19 & & 3001 & V 905 Cen & 3275 & 7.98 & \\
\hline 3058 & $\mathrm{BD}+02^{\circ} 2446$ & 4440: & 9.69 & & 3066 & HD 100764 & 4600 & $7.87^{\mathrm{d}}$ & & 3083 & RR Mus & 3090 & 4.86 & \\
\hline 3141 & DD Cru & 3545 & 6.29 & & 3156 & $\mathrm{BD}+71^{\circ} 600$ & 4160 & 9.58 & & 3215 & IR12173-5839 & 2865 & 6.75 & \\
\hline
\end{tabular}


Table A.1. continued

\begin{tabular}{|c|c|c|c|c|c|c|c|c|c|c|c|c|c|c|}
\hline Ent. & Name & $T_{\text {eff }}$ & $m_{\mathrm{bol1}}$ & Ph. & Ent. & Name & $T_{\text {eff }}$ & $m_{\text {bol1 }}$ & $\mathrm{Ph}$. & Ent. & Name & $T_{\text {eff }}$ & $m_{\text {bol1 }}$ & $\mathrm{Ph}$. \\
\hline 3222 & RS Mus & 2615 & 5.11 & & 3227 & S Cen & 3270 & 5.47 & & 3236 & SS Vir & 2560 & 3.69 & 0.0 \\
\hline 3246 & V 927 Cen & 2890 & 6.53 & & 3283 & Y CVn & 2760 & 2.43 & & 3284 & IR12444-5925 & 2420 & 4.72 & \\
\hline 3286 & RU Vir & 2100 & $3.92^{\mathrm{a}}$ & 0.01 & 3286 & RU Vir & 1945 & $4.55^{\mathrm{a}}$ & 0.46 & 3291 & RX Cru & 2650 & 4.58 & \\
\hline 3298 & HD 111908 & 4235: & 8.52 & & 3310 & V Cru & 3075 & 5.67 & 0.81 & 3313 & RY Dra & 2810 & 3.42 & \\
\hline 3319 & TT CVn & 3865 & 7.69 & & 3335 & HD 113801 & 5000 & 8.01 & & 3368 & T Mus & 2830 & 5.06 & \\
\hline 3374 & UX Cen & 2925 & 4.99 & & 3379 & $\mathrm{BD}+04^{\circ} 2735$ & 4915 & 9.67 & & 3405 & V 971 Cen & 3425 & 6.33 & \\
\hline 3412 & RV Cen & 2865 & 4.36 & 0.15 & 3412 & RV Cen & 2680 & 4.87 & 0.53 & 3456 & NSV 6507 & 2920 & 4.36 & \\
\hline 3469 & HD 122547 & 4250 & 8.89 & & 3471 & U Cir & 3075 & 6.17 & & 3481 & V 996 Cen & 2695 & 3.79 & \\
\hline 3492 & RS Lup & 3000 & 6.67 & & 3510 & Z Lup & 2655 & 5.17 & & 3533 & V 553 Cen & 6150 & 8.06 & \\
\hline 3533 & V 553 Cen & 5650 & 8.35 & & 3548 & V Lup & 3225 & 5.50 & & 3558 & NSV 6912 & 3360 & 8.44 & \\
\hline 3562 & S Aps & 5115 & $8.30^{\mathrm{e}}$ & $\max$ & 3569 & $\mathrm{X} \operatorname{Tr} \mathrm{A}$ & 2710 & 2.60 & & 3572 & AS Cir & 2420 & 4.80 & \\
\hline 3586 & $\mathrm{BD}+30^{\circ} 2637$ & 5130 & 9.41 & & 3591 & $\mathrm{BD}+83^{\circ} 442$ & 5115 & 9.48 & & 3594 & U Aps & 2605 & 4.95 & \\
\hline 3594 & U Aps & 2665 & 4.57 & & 3606 & HM Lib & 4725 & 7.14 & & 3614 & NSV 7110 & 3935 & 8.95 & \\
\hline 3652 & V CrB & 2090 & 4.83 & 0.1 & 3665 & RR Her & 3055 & 6.19 & & 3672 & HD 145777 & 4245 & 9.12 & \\
\hline 3687 & RT Nor & 5615 & 9.30 & & 3697 & V377 Nor & 3200 & 5.03 & & 3698 & V Oph & 3010 & 4.56 & 0.94 \\
\hline 3704 & NSV 7765 & 4600 & 9.55 & & 3707 & NSV 7820 & 5625 & 8.08 & & 3720 & SU Sco & 2655 & 4.19 & \\
\hline 3731 & V TrA & 3025 & 5.76 & & 3735 & NSV 7869 & 4525 & 9.09 & $\min$ & 3756 & T Ara & 3165 & 5.75 & \\
\hline 3762 & V 901 Sco & 2550 & 5.83 & & 3774 & SZ Ara & 3295 & 7.12 & & 3795 & HD 156074 & 4720 & 7.33 & \\
\hline 3799 & NSV 8476 & 3220 & 5.80 & & 3808 & V 1079 Sco & 2510 & 4.49 & & 3816 & $\mathrm{CD}-36^{\circ} 11460$ & 4375 & 7.44 & \\
\hline 3820 & V 522 Oph & 2425 & 5.00 & & 3827 & V 644 Sco & 2615 & 5.16 & & 3837 & TW Oph & 2440 & 3.36 & \\
\hline 3842 & NSV 9082 & 3740 & 7.63 & & 3854 & TT Sco & 2430 & 4.64 & & 3861 & V Pav & 2545 & 3.42 & \\
\hline 3864 & V 450 Sco & 2915 & 6.23 & & 3875 & SZ Sgr & 3220 & 4.94 & & 3878 & SX Sco & 2785 & 4.58 & \\
\hline 3879 & $\mathrm{BD}+17^{\circ} 3325$ & 4835 & 8.01 & & 3901 & V 781 Sgr & 2990 & 4.78 & & 3912 & HD 163838 & 4365 & 10.14 & \\
\hline 3921 & T Dra & 1850 & $4.94^{\mathrm{f}}$ & 0.95 & 3933 & V $4378 \mathrm{Sgr}$ & 3255 & 5.32 & & 3938 & W CrA & 3945 & 7.76 & \\
\hline 3947 & V $4380 \mathrm{Sgr}$ & 2725 & 6.40 & & 3950 & WX CrA & 4805 & $9.19^{g}$ & & 3957 & NSV 10269 & 3925 & 8.75 & \\
\hline 3960 & V $1280 \mathrm{Sgr}$ & 2620 & 4.75 & 0.02 & 3973 & HIC 89239 & 5080 & 9.77 & & 3982 & RS Tel & 5800 & 9.21 & $\max$ \\
\hline 3982 & RS Tel & 5800 & $11.13^{\mathrm{a}}$ & $\min$ & 3987 & ES Ser & 2500 & 4.73 & & 3992 & FO Ser & 3345 & 5.74 & \\
\hline 3999 & GU Sgr & 4880 & 9.20 & $\max$ & 4002 & HIC 90199 & 4210 & 9.14 & & 4021 & HD 170282 & 4115 & 8.51 & \\
\hline 4025 & SS Sgr & 2990 & 5.49 & & 4032 & TY Oph & 2680 & 4.81 & & 4038 & T Lyr & 2310 & 3.62 & \\
\hline 4052 & RX Sct & 3010 & 4.48 & & 4086 & RV Sct & 3335 & 6.04 & & 4089 & HK Lyr & 2620 & 4.89 & \\
\hline 4094 & HD 173409 & 5640 & 9.43 & & 4098 & V CrA & 5800 & 8.70 & $\max$ & 4098 & $\mathrm{~V}$ CrA & 5800 & $9.36^{\mathrm{a}}$ & int \\
\hline 4111 & DR Ser & 2650 & 5.16 & & 4121 & S Sct & 2755 & 3.63 & & 4138 & T Sct & 3000 & 5.71 & \\
\hline 4145 & V $4152 \mathrm{Sgr}$ & 4815 & 8.80 & & 4147 & UV Aql & 2700 & 4.79 & & 4152 & VX Aql & 2785 & 5.78 & \\
\hline 4159 & $\mathrm{BD}+10^{\circ} 3764$ & 3265 & 4.78 & & 4164 & V Aql & 2525 & 3.10 & & 4179 & HD 178316 & 4150 & 9.26 & \\
\hline 4181 & SV Sge & 4010 & 8.27 & & 4194 & V $1445 \mathrm{Aql}$ & 3435 & 7.88 & & 4208 & V 553 Lyr & 2965 & 6.62 & \\
\hline 4217 & CG Vul & 2685 & 4.71 & & 4229 & V 1942 Sgr & 2960 & 4.10 & & 4241 & U Lyr & 2440 & 5.43 & 0.06 \\
\hline 4247 & NSV 11960 & 5590 & 6.15 & & 4263 & NSV 11995 & 3905 & 9.10 & & 4302 & UX Dra & 3090 & 3.16 & \\
\hline 4307 & AW Cyg & 2795 & 5.11 & & 4333 & AQ Sgr & 2790 & 3.83 & & 4373 & V $391 \mathrm{Aql}$ & 2655 & 6.22 & \\
\hline 4390 & HD $92626^{\mathrm{b}}$ & 4400 & 6.57 & & 4415 & TT Cyg & 2825 & 4.99 & & 4454 & UW Sgr & 3225 & 6.59 & \\
\hline 4485 & HD 187216 & 3850 & 8.47 & & 4524 & HD 187861 & 4905 & 8.62 & & 4567 & HD 188934 & 3960 & 7.58 & \\
\hline 4581 & AX Cyg & 2655 & 4.56 & & 4595 & V $1468 \mathrm{Aql}$ & 3455 & $7.83^{\mathrm{h}}$ & & 4598 & V $1469 \mathrm{Aql}$ & 3800 & 6.21 & \\
\hline 4616 & BF Sge & 2935 & 6.36 & & 4619 & V 2102 Cyg & 3350 & 6.73 & & 4653 & X Sge & 2630 & 5.07 & \\
\hline 4712 & AY Cyg & 2640 & 5.90 & & 4714 & SV Cyg & 2600 & 5.01 & & 4716 & RY Cyg & 2790 & 5.91 & \\
\hline 4758 & RS Cyg & 3100 & 4.00 & & 4774 & RT Cap & 2480 & 3.80 & & 4784 & NSV 12948 & 4555 & 7.99 & \\
\hline 4806 & WX Cyg & 3305 & 5.07 & 0.0 & 4806 & WX Cyg & 3140 & 5.44 & 0.27 & 4817 & U Cyg & 2650 & 4.23 & 0.28 \\
\hline 4817 & U Cyg & 2530 & 4.16 & 0.57 & 4848 & HH Del & 2965 & 7.72 & & 4851 & V 744 Cyg & 3325 & 6.88 & \\
\hline 4873 & BI Cap & 3440 & 7.99 & & 4923 & V 778 Cyg & 3320 & 6.50 & & 4939 & V Cyg & 1885 & 3.06 & 0.1 \\
\hline 4939 & V Cyg & 1875 & 3.41 & 0.27 & 4947 & HD 197604 & 4165 & 8.72 & & 4972 & HD 198140 & 3890 & 9.54 & \\
\hline 4978 & HD 198269 & 4280 & 7.62 & & 4989 & V 1862 Cyg & 2610 & 5.64 & & 5147 & HD 201266 & 3480 & 8.05 & \\
\hline 5227 & HD 202851 & 4780 & 9.28 & & 5228 & $\mathrm{~T}$ Ind & 2990 & 3.69 & & 5230 & $\mathrm{BD}+02^{\circ} 4338$ & 4240 & 9.11 & \\
\hline 5239 & Y Pav & 2945 & 3.28 & 0.06 & 5239 & Y Pav & 2865 & 3.51 & 0.84 & 5265 & YY Cyg & 2815 & 5.70 & \\
\hline 5358 & V 1426 Cyg & 1975 & $4.08^{\mathrm{a}}$ & 0.85 & 5358 & V 1426 Cyg & 1875 & 4.21 & 0.15 & 5406 & S Cep & 2240 & 3.05 & 0.05 \\
\hline 5406 & S Cep & 2095 & 3.25 & 0.5 & 5408 & BU Ind & 3455 & 8.32 & & 5418 & V 460 Cyg & 2950 & 3.20 & \\
\hline 5420 & RR Ind & 2985 & 6.46 & & 5425 & RV Cyg & 2675 & 3.46 & 0.44 & 5494 & LW Cyg & 2580 & 4.75 & \\
\hline 5495 & V 413 Cyg & 2815 & 5.17 & & 5496 & RX Peg & 2935 & 5.27 & & 5549 & $\mathrm{U}$ Aqr & $5000^{\mathrm{i}}$ & 10.26: & $\max$ \\
\hline 5549 & $\mathrm{U}$ Aqr & 5000 & $10.63:^{\mathrm{f}}$ & $\operatorname{dip}$ & 5560 & V 378 Lac & 2995 & 7.40 & & 5561 & HP Peg & 4190 & 8.09 & \\
\hline 5570 & RZ Peg & 3245 & 5.90 & 0.87 & 5570 & RZ Peg & 2605 & $5.22^{\mathrm{a}}$ & 0.20 & 5577 & CT Lac & 2555 & $5.46^{\mathrm{a}}$ & \\
\hline 5677 & V 451 Cep & 2790 & 5.64 & & 5714 & HIC 112306 & 3660 & 8.48 & & 5719 & DG Cep & 2985 & 4.81 & \\
\hline 5728 & TX Lac & 3195 & 7.25 & & 5761 & HD 216649 & 4360 & 10.16 & & 5774 & TV Lac & 3290 & 6.25 & \\
\hline 5791 & VY And & 2650 & 6.16 & & 5822 & HD 218851 & 4770 & 8.81 & & 5823 & HD 218875 & 4555 & 8.96 & \\
\hline 5879 & EW And & 2945 & 6.09 & $\min$ & 5903 & ST And & 2825 & 6.56 & & 5928 & TX Psc & 3125 & 2.41 & \\
\hline 5937 & HD 223392 & 4315 & 7.80 & & 5976 & WZ Cas & 3095 & 3.59 & 0.62 & 5980 & HD 224959 & 4775 & 9.26 & \\
\hline \multirow[t]{5}{*}{5987} & SU And & 2905 & 5.50 & & $\mathrm{c} 18$ & $\mathrm{BD}+29^{\circ} 95$ & 4200: & 9.82 & & & HD 26 & 5250 & 7.86 & \\
\hline & XX Cam & 6900 & 6.14 & & & XX Cam & 6900 & 6.36 & & & GP Ori & 3010 & 5.06 & \\
\hline & AFGL 799 & 1680: & $6.69^{\mathrm{a}}$ & & & SU Tau & 6900 & $7.58^{\mathrm{f}}$ & & & FU Mon & 3345 & 4.66 & $\max$ \\
\hline & FU Mon & 2825 & 5.14 & 0.27 & & NSV $3024^{\mathrm{b}}$ & 4900 & 5.96 & & & V 688 Mon & 1670: & $5.25^{\mathrm{a}}$ & 0.09 \\
\hline & UX Vol & 2725 & 5.70 & $\max$ & c1633 & V 496 Car & 3270 & 4.01 & & & IR10127-6026 & 3520 & 5.63 & \\
\hline S674 & IR10164-6044 & 2730 & 6.60 & & $\mathrm{~S} 816$ & UY Cen & 2815 & 3.64 & & S830 & AM Cen & 2715 & 4.72 & \\
\hline \multirow[t]{2}{*}{ S904 } & VY Aps & 2850 & 5.61 & & & $\mathrm{R}$ CrB & 6100 & $5.62^{\mathrm{f}}$ & & & $\mathrm{RT} \operatorname{Tr} \mathrm{A}$ & 6500 & $9.19^{j}$ & 0.0 \\
\hline & RT $\operatorname{Tr} A$ & 5550 & $9.38^{\mathrm{j}}$ & 0.2 & & RT $\operatorname{Tr} A$ & 5200 & $9.65^{\mathrm{j}}$ & 0.6 & & $\mathrm{RT} \operatorname{Tr} \mathrm{A}$ & 6100 & $9.43^{\mathrm{j}}$ & 0.8 \\
\hline \multirow[t]{4}{*}{ S935 } & IR16382-5727 & 2720 & 6.66 & & & OP Her & 3390 & 3.01 & & & FX Ser & 2050 & $5.02^{\mathrm{a}}$ & $\max$ \\
\hline & RY Sgr & 6900 & $5.67^{\mathrm{f}}$ & $\max$ & & CY Cyg & 2690 & 5.16 & & & NSV 13571 & 4800 & 7.71 & \\
\hline & LP And & 2040 & $4.19^{\mathrm{a}}$ & & & rho Cas & 4600 & 3.66 & & & HD 76115 & 4900 & 8.19 & \\
\hline & BQ Oct & 3520 & 4.36 & & & RU Aqr & 3270 & 6.07 & & & AC Her & 5850 & $7.57^{\mathrm{k}}$ & \\
\hline
\end{tabular}

${ }^{\mathrm{a}}$ Circumstellar extinction detected, ${ }^{\mathrm{b}}$ A barium star, ${ }^{\mathrm{c}}$ Silicate-type excess at $10 \mu \mathrm{m} ; m_{\text {bol2 }}=6.17,{ }^{\mathrm{d}} \mathrm{IR}$ excess; $m_{\text {bol2 }}=8.51$, ${ }^{\mathrm{e}} \mathrm{IR}$ excess; $m_{\text {bol } 2}=8.49,{ }^{\mathrm{f}}$ IR excess, ${ }^{\mathrm{g}} \mathrm{IR}$ excess; $m_{\text {bol } 2}=9.64,{ }^{\mathrm{h}}$ Silicate-type excess at $10 \mu \mathrm{m} ; m_{\text {bol } 2}=8.04,{ }^{\mathrm{i}}$ IR excess or HC1: $4920 \mathrm{~K},{ }^{\mathrm{f}}$ IR excess, ${ }^{\mathrm{j}} \mathrm{sg} ; \mathrm{CWB},{ }^{\mathrm{k}}$ IR excess; a RV Tau-star. 